\title{
GEOMETRIC FLOWS FOR THE TYPE IIA STRING 1
}

\author{
Teng Fei, Duong H. Phong, Sebastien Picard, and Xiangwen Zhang
}

\begin{abstract}
A geometric flow on 6-dimensional symplectic manifolds is introduced which is motivated by supersymmetric compactifications of the Type IIA string. The underlying structure turns out to be SU(3) holonomy, but with respect to the projected Levi-Civita connection of an almost-Hermitian structure. The short-time existence is established, and new identities for the Nijenhuis tensor are found which are crucial for Shi-type estimates. The integrable case can be completely solved, giving an alternative proof of Yau's theorem on Ricci-flat Kähler metrics. In the non-integrable case, models are worked out which suggest that the flow should lead to optimal almost-complex structures compatible with the given symplectic form.
\end{abstract}

\section{Introduction}

There has been a remarkable confluence in recent years between high energy physics, more specifically unified string theories, and special geometry. The earliest and particularly influential development was the 1985 recognition by Candelas, Horowitz, Strominger, and Witten [8] of Calabi-Yau manifolds as supersymmetric compactifications of the heterotic string. The importance of geometry in physical laws at their most fundamental level has of course been long recognized with electromagnetism, general relativity, and gauge theories. The key new feature here is the requirement that the 6-dimensional internal manifold have a special geometric structure, in this case a complex structure together with a holomorphic section of the canonical bundle. This requirement is equivalent to the manifold having SU(3) holonomy, and can be traced back to supersymmetry. Since then, the class of Calabi-Yau solutions has been enlarged in many directions. On one hand, the Calabi-Yau condition can be extended to the Hull-Strominger system for conformally balanced metrics [32, 44, 23, 24, 40, 41, 25, 21, 18]. On the other hand, it emerged from the mid 1990's that string theories, which are 10-dimensional and of which there are five, can be unified themselves into another theory, namely M Theory, one of whose limits is 11dimensional supergravity [31, 48]. The compactification of an 11-dimensional space-time to a more familiar 4-dimensional space time results in a 7-dimensional internal space, and the role of Calabi-Yau manifolds is assumed in this case by manifolds with $\mathrm{G}_{2}$ or $\operatorname{Spin}(7)$ holonomy [1, 28].

While the full string theories have been conjectured to merge ultimately into a single M Theory, this requires highly non-trivial dualities, and their low-energy approximations

\footnotetext{
${ }^{1}$ Work supported in part by the National Science Foundation Grants DMS-1855947 and DMS-1809582.
} 
and geometric settings can be quite different. A common feature of their supersymmetric compactifications is a metric satisfying a curvature condition as well as a cohomological condition. In Kähler geometry, these are characteristic features of the notion of canonical metric, of which the Calabi-Yau condition is the prime example. Thus the general case can be viewed as a search for canonical metrics in non-Kähler geometry. The compactifications discussed above arise from three of the string theories, which are the Type I theory and the two heterotic string theories. The other two string theories are the Type IIA and the Type IIB theories. There is an immense literature on their supersymmetric compactifications, but some attractive mathematical formulations can be found in Grana et al. [27] and Tomasiello [45], and the study of the most basic examples was begun in Tseng and Yau [50, 51, 52. The geometric realm for the Type IIB equation is that of complex geometry, albeit non-Kähler, and we described a geometric flow approach to it in [19]. The main goal of the present paper is to present a geometric flow approach to the Type IIA equation.

The Type IIA equation is of particular interest, because of all string theory compactifications, its geometric setting is unique in being that of symplectic geometry instead of complex geometry. More specifically, let $M$ be a compact 6-dimensional manifold, equipped with a symplectic form $\omega$, that is, a closed non-degenerate 2 -form. Recall that on any oriented 6-manifold, Hitchin [32] had shown how to associate to a non-degenerate 3 -form $\varphi$ an almost-complex structure $J_{\varphi}$. In the Type IIA equation, a symplectic form $\omega$ is given, so it makes sense to consider the condition of primitiveness for $\varphi$ with respect to $\omega$. Explicitly, this is the condition $\Lambda \varphi=0$, where

$$
\Lambda: A^{k}(M) \rightarrow A^{k-2}(M)
$$

is the usual Hodge operator of contracting with $\omega$. As shown in $\S 4.1$ below, the symplectic form $\omega$ is invariant with respect to the almost-complex structure $J_{\varphi}$ when $\varphi$ is primitive. We obtain then a Hermitian form

$$
g_{\varphi}(X, Y)=\omega\left(X, J_{\varphi} Y\right)
$$

which becomes a Hermitian metric under the open condition that it be strictly positive. Thus we obtain an almost-Kähler 3 -fold $\left(M, \omega, J_{\varphi}, g_{\varphi}\right)$ with the additional requirement that $\varphi$ be primitive and $J_{\varphi}$ arise from $\varphi$ by the above construction. When $\varphi$ is also closed, we shall refer to such a structure as a "Type IIA geometry".

Let $\rho_{A}$ be now the Poincaré dual of a given finite linear combination of special Lagrangians calibrated by $\varphi$. Then the Type IIA equation is the following system of equations for a real-valued primitive 3 -form $\varphi$

$$
d \Lambda d\left(|\varphi|^{2} \star \varphi\right)=\rho_{A}, \quad d \varphi=0, \quad g_{\varphi}>0 .
$$

Here $\star$ is the Hodge star operator and $|\varphi|$ the norm of $\varphi$ with respect to the metric $g_{\varphi}$. 
As in the case of the other string theories, the Type IIA equations as written in (1.3) involve, besides the open condition $g_{\varphi}>0$, a curvature-type equation and the cohomological constraint $d \varphi=0$. In order to enforce this cohomological constraint without invoking any particular Ansatz, we introduce the following geometric flow of 3-forms $\varphi$,

$$
\partial_{t} \varphi=d \Lambda d\left(|\varphi|^{2} \star \varphi\right)-\rho_{A},
$$

for any closed and primitive initial data $\varphi_{0}$ with $g_{\varphi_{0}}>0$. Since the right hand side is closed, the flow preserves the closedness condition. It can also be verified to preserve the primitiveness property of $\varphi$. Thus it is a flow of Type IIA geometries, whose stationary points would give solutions of the Type IIA equation without recourse to any Ansatz. We shall refer to (1.4) as the Type IIA flow. The idea of preserving the closedness of a form by a flow was introduced by Bryant [5] in the Laplacian flow for $G_{2}$ structures. More recently, it was applied in [38, 39, 19, 17, 37] to the construction of geometric flows which preserve the conformally balanced condition in the Hull-Strominger system and the Type IIB equation. The geometric flow approach was particularly appropriate there, as it allowed to bypass the absence of a $\partial \bar{\partial}$-lemma in non-Kähler geometry.

The main goal of this paper is to start an in-depth study of the Type IIA flow. Except for the original formulation, we shall restrict to the most basic source-free case $\rho_{A}=0$. Despite its very simple formulation (1.4), the flow turns out to be highly non-trivial and to present many new difficulties specific to symplectic geometry:

- The first is that the Type IIA equation is not elliptic. This difficulty was wellrecognized in the works of Tseng and Yau [52] and Tseng and Wang [49], and led them to consider instead some 4th-order equations. However, 4th-order equations are complicated, and the closedness constraint on the 3 -form $\varphi$ would have to be imposed separately. Thus it appears still preferable to confront the specific difficulties of the Type IIA flow. They originate in any case from the geometric assumption of a given symplectic structure, which is fundamental in symplectic geometry.

- The second may be appreciated by comparing the flow of almost-complex structures $J_{\varphi}$ in the Type IIA flow with the gradient flow of the Blair-Ianus functional on a symplectic manifold. The Blair-Ianus functional is the $L^{2}$ norm of the Nijenhuis tensor [4]. Its gradient flow was called the anti-complexified Ricci flow by Lê and Wang [33], who also established its short-time existence. However, this flow has proved to be difficult to use, because neither the corresponding Nijenhuis tensor nor curvature evolves there by parabolic equations. For the Type IIA flow to be viable, it has to overcome such difficulties.

- The third difficulty is more technical, but still serious. The Type IIA flow of 3forms $\varphi$ induces a flow of metrics $g_{\varphi}$ which will be one of the main tasks of this paper to determine explicitly. The simplest case is when the initial almost-complex structure is integrable. It turns out that the Type IIA flow preserves the integrability condition, and becomes equivalent to the dual Anomaly flow introduced in [20]. Using the techniques 
there as well as in [42, 43], it gives a new proof of Yau's [53] celebrated theorem on the existence of Kähler Ricci-flat metrics. Thus the difficult case is the case of non-integrable almost-complex structures. There we shall see that the flow of metrics in the Type IIA flow is conformally equivalent to a perturbation of the Ricci flow by first-order terms involving the Nijenhuis tensor. In this respect, it is analogous to Bryant's $G_{2}$ Laplacian flow, which was shown relatively recently by Lotay and Wei [35] to be a perturbation of the Ricci flow by first-order terms involving the torsion tensor. However, the long-time behavior of the $G_{2}$ flow remains at this moment a subject of extensive research [34, 2].

Despite these difficulties, we shall find that the Type IIA flow is particularly rich, reflecting its unique position at the crossroads of symplectic geometry, complex geometry, and unified string theories. This rich structure will be much in evidence in the results described below. Furthermore, examples suggest that even when the flow develops singularities, it may be possible in some cases to continue the flow of the Nijenhuis tensor. Thus, besides the original motivation from string theory and interest in its stationary points, the flow should also be useful in finding optimal almost-complex structures.

\section{Main results}

We describe now our main results. Throughout this section, $M$ is a compact 6 -dimensional manifold equipped with a symplectic form $\omega$. Given a primitive 3-form $\varphi$, we denote by $J_{\varphi}$ the almost-complex structure defined by Hitchin [32], and by $g_{\varphi}$ the corresponding Hermitian form, which we assume is a metric.

\subsection{A Laplacian flow formulation}

Our first result is an alternative formulation of the Type IIA flow:

Theorem 1 The Type IIA flow defined in (1.4) can be rewritten as the following flow

$$
\partial_{t} \varphi=-d d^{\dagger}\left(|\varphi|^{2} \varphi\right)+2 d\left(|\varphi|^{2} N^{\dagger} \cdot \varphi\right)-\rho_{A}
$$

where $d^{\dagger}$ is the adjoint of the operator $d$ with respect to the metric $g_{\varphi}$, and $N^{\dagger} i s$ the operator from $\Lambda^{3}(M)$ to $\Lambda^{2}(M)$ defined by

$$
\left(N^{\dagger} \cdot \varphi\right)_{k j}=N^{\mu}{ }_{j}{ }^{\lambda} \varphi_{\mu k \lambda}-N_{k}^{\mu}{ }^{\lambda} \varphi_{\mu j \lambda} .
$$

Here $N_{\gamma \beta}^{m}$ is the Nijenhuis tensor of $J_{\varphi}$, and indices are raised using the metric $g_{\varphi}$.

We note that, up to the factor of $|\varphi|^{2}$, the first terms on the right-hand side of the Type IIA flow are the same as in the standard heat equation. Up to sign, they are also reminiscent of Bryant's $G_{2}$ Laplacian flow. However, the terms involving the Nijenhuis tensor are also of leading order and account for a wide range of different phenomena.

Henceforth, we assume that the source $\rho_{A}$ is 0 , unless stated explicitly otherwise. 


\subsection{The short-time existence of the Type IIA flow}

When a flow is not strictly parabolic, even its short-time existence can be a difficult question. Two powerful tools developed over the years for this issue have been the reparametrization method of DeTurck [14] and the Hamilton-Nash-Moser theorem [29], a combination of which has been applied successfully to many important flows, such as the Ricci flow [29], the $G_{2}$ Laplacian flow [5, 6], and the anti-complexified Ricci flow [33]. The fundamental new difficulty in the Type IIA flow is that there is a given symplectic form $\omega$. It is not hard to see that reparametrizations by symplectomorphisms do not improve the parabolicity of the flow, while more general reparametrizations lead to a coupled flow of both metrics and symplectic forms. Thus a first major task in this paper is to establish the following theorem:

Theorem 2 Let $(M, \omega)$ be a compact 6-dimensional symplectic manifold. Then for any $\varphi_{0}$ which is a smooth positive, primitive, and closed 3-form, the source-free Type IIA flow (1.4) with initial value $\varphi_{0}$ admits a unique and smooth solution on some time interval $[0, T)$ with $T>0$. Furthermore, $\varphi$ continues to be positive, primitive and closed at all times.

While the theorem deals only with the short-time existence of the flow, the proof requires a rather deep probe of the structure of the flow and several new elements which are also useful elsewhere:

The first element is the behavior of the coupled flows mentioned above. It turns out that these coupled flows admit natural parabolic regularizations, which reduce to the desired flow for primitiveness initial data. While primitiveness was a requirement in the solution $\varphi$ of the Type IIA equation, it may not have been anticipated that it would play such a central role for the very existence of the flow.

The second feature permeates the rest of the paper, and is the underlying Type IIA geometry. In the present context, it allows us to recapture the flow of the forms $\varphi$ from the flow of the metrics $\tilde{g}_{\varphi}=|\varphi|^{2} g_{\varphi}$, even though, pointwise, there is an ambiguity in determining $\varphi$ from $\tilde{g}_{\varphi}$. Since we shall have to analyze in detail the flow of metrics in order to obtain Shi-type estimates and long-time existence criteria, it is simplest to deduce the uniqueness part of Theorem 2 from the corresponding uniqueness theorems for the flow of metrics. The flow of $\check{g}_{\varphi}=|\varphi|^{-2} g_{\varphi}$ turns out to be a perturbation of the Ricci flow by terms of first order. In general, integrability operators are not stable under first-order terms perturbations. However, the underlying Type IIA geometry is what allows us to modify the Bianchi operator used by Hamilton [29] for the Ricci flow into an integrability operator for the flow of $\check{g}_{\varphi}$. From there we can establish the uniqueness for $\check{g}_{\varphi}$, and from there the uniqueness in Theorem 2 . 


\subsection{Type IIA geometry}

We have stressed that the underlying structure for the Type IIA flow is Type IIA geometry, as defined in the Introduction, and which is more special than just a symplectic structure on a 6-manifold. The holonomy, curvature, and Nijenhuis tensor in Type IIA geometry have rather special properties, which play an important role in every aspect of the Type IIA flow. We list here some properties which are applied repeatedly in the paper and are the easiest to describe, but we expect others to emerge and prove their worth in time:

Theorem 3 Let $(M, \omega, \varphi)$ be a Type IIA geometry, and $g_{\varphi}$ the corresponding metric. Set

$$
\tilde{g}_{\varphi}=|\varphi|^{2} g_{\varphi}
$$

Let $\mathfrak{D}$ and $\tilde{\mathfrak{D}}$ be the projected Levi-Civita connections of $g_{\varphi}$ and $\tilde{g}_{\varphi}$ respectively, $\Omega=$ $\varphi+i J_{\varphi} \varphi$, and $|\Omega|_{\tilde{g}_{\varphi}}$ the norm of $\Omega$ with respect to $\tilde{g}_{\varphi}$. Then

(a) $\tilde{\mathfrak{D}}\left(\frac{\Omega}{|\Omega|_{\tilde{g}_{\varphi}}}\right)=0$. Thus $\left(M, \tilde{g}_{\varphi}\right)$ has holonomy in $S U(3)$, but with respect to the connection $\tilde{\mathfrak{D}}$.

(b) $\mathfrak{D}^{0,1} \Omega=0$, so $\Omega$ is formally holomorphic, even when $J_{\varphi}$ is not integrable.

(c) The Nijenhuis tensor has only 6 independent components.

\subsection{The flow of metrics in the Type IIA flow}

Next, we can describe the flow of the 3 -forms $\varphi$ and metrics $g_{\varphi}$ in terms of curvature:

Theorem 4 Let $\varphi$ be a smooth positive, primitive and closed 3-form evolving by the sourcefree Type IIA flow. Set $u=\log |\varphi|^{2}$.

(a) The flow of $\varphi$ is given by

$$
\partial_{t} \varphi_{i a b}=e^{2 u} \sum_{\text {cyc } i, a, b}\left(\varphi_{s a b}\left(\tilde{\mathfrak{D}}_{i}+u_{i}\right) u^{s}+2 \varphi_{\text {sta }}\left(N_{p}^{s t}{ }_{p} \tilde{\mathfrak{T}}^{p}{ }_{i b}-\frac{u^{s}}{2} \tilde{\mathfrak{T}}^{t}{ }_{i b}+\left(\tilde{\mathfrak{D}}_{i}+u_{i}\right) N_{b}^{s t}\right)\right),
$$

where $\tilde{\mathfrak{T}}$ is the torsion tensor of the connection $\tilde{\mathfrak{D}}$.

(b) The flow of $\tilde{g}_{\varphi}$ is given by

$$
\begin{aligned}
\left(\partial_{t} \tilde{g}_{\varphi}\right)_{i j}= & e^{2 u}\left[-2 \tilde{R}_{i j}-2\left(\tilde{\nabla}^{2} u\right)_{i j}+4 u^{s}\left(N_{i s j}+N_{j s i}\right)+u_{i} u_{j}-u_{J i} u_{J j}-4\left(N_{-}^{2}\right)_{i j}\right. \\
& \left.+\left(|d u|_{\tilde{g}_{\varphi}}^{2}+|N|_{\tilde{g}}^{2}\right)\left(\tilde{g}_{\varphi}\right)_{i j}\right],
\end{aligned}
$$

where $\tilde{\nabla}$ is the Levi-Civita connection of $\tilde{g}_{\varphi}$. 
Note that $u$ is determined by $\tilde{g}_{\varphi}$, so the right hand side of (2.5) involves only tensors determined by $\tilde{g}$, and the equation is a self-contained flow for $\tilde{g}_{\varphi}$. Furthermore, the equation for $\varphi$ can be viewed as a linear ODE of $\varphi$ whose coefficients are tensors determined by $\tilde{g}_{\varphi}$. Thus $\varphi$ is completely determined once $\tilde{g}_{\varphi}$ is determined. As we noted before, this is to be contrasted with the problem of having to resolve an ambiguity if we just try to recapture $\varphi$ from $g_{\varphi}$ at each fixed time. It may be worth observing that the ambiguity in recapturing $\varphi$ from $g_{\varphi}$ pointwise in time is reminiscent of the ambiguity in defining the angle in the special Lagrangian equation. It would be interesting to investigate if the angle in the special Lagrangian equation can be recaptured by a mechanism similar to the above Theorem 4 for the Type IIA flow.

\subsection{An integrability operator for the flow of metrics}

The flow (2.5) is reminiscent of the Ricci flow, except for the term $\left(\tilde{\nabla}^{2} u\right)_{i j}$ which can normally be eliminated by a reparametrization. But as we noted in the above discussion of Theorem 2, a reparametrization would create other difficulties since it would change the given symplectic form $\omega$. To bypass this difficulty, we make instead a conformal change

$$
\check{g}_{i j}=|\varphi|^{-2}\left(g_{\varphi}\right)_{i j}
$$

and find that $\check{g}_{i j}$ evolves by

$$
\begin{aligned}
\partial_{t} \check{g}_{i j}= & e^{\frac{3}{2} u}\left[-2 \check{R}_{i j}+\frac{3}{2} u_{i} u_{j}-u_{J i} u_{J j}+4 u^{k}\left(N_{i k j}+N_{j k i}\right)-4\left(N_{-}^{2}\right)_{i j}\right. \\
& \left.+\frac{1}{2}\left(|d u|_{\check{g}}^{2}+|N|_{\check{g}}^{2}\right) \check{g}_{i j}\right],
\end{aligned}
$$

For the purpose of completing Theorem 2, we are particularly interested in the uniqueness of this flow. For the classical Ricci flow, both the short-time existence and uniqueness were established by Hamilton [29] using a version of the Nash-Moser theorem. This version, often referred to as the Hamilton-Nash-Moser theorem, requires an integrability condition, which was provided by the Bianchi identity in the case of the Ricci flow. More precisely, let $L_{0}$ be the operator defined by

$$
L_{0}: \operatorname{Sym}^{2}(T M) \ni S_{i j} \rightarrow\left(L_{0}(S)\right)_{j}=2 \check{g}^{i k} \check{\nabla}_{k} S_{i k}-\check{g}^{i k} \check{\nabla}_{j} S_{i k} \in A^{1}(M)
$$

Then $L_{0}\left(-2 \check{R}_{i j}\right)=0$, which is the desired integrability condition. Now the flow (2.7) differs from the Ricci flow by first-order terms, so the Bianchi identity is no longer applicable as an integrability condition. In general, it is by no means clear whether a given first-order perturbation would still allow an integrability condition. So it is again a manifestation of the deep structure of Type IIA geometry that this can be done in this case: 
Theorem 5 Let $S_{i j}$ be the symmetric 2-tensor defined by writing the flow (2.7) as

$$
\partial_{t} \check{g}_{i j}=e^{\frac{3}{2} u}\left(-2 \check{R}_{i j}+S_{i j}\right) .
$$

(a) Define the operator $Z$ by

$$
Z: \operatorname{Sym}^{2}(T M) \ni S_{i j} \rightarrow(Z(S))_{j}=-\frac{4}{3} u_{j} \check{g}^{i k} S_{i k}+2 u^{s} S_{j s}-4 N^{s t}{ }_{j} S_{s t} \in A^{1}(M) .
$$

If along the flow, the metric $\check{g}$ arises from a Type IIA geometry, then the following operator $L$ is an integrability operator for the flow 2.7) in the sense that

$$
\operatorname{Sym}^{2}(T M) \ni \check{g}_{i j} \rightarrow L\left(\check{g}_{i j}\right):=\left(L_{0}+Z\right)\left(e^{\frac{3}{2} u}\left(-2 \check{R}_{i j}+S_{i j}\right)\right)
$$

is of order 1 in $\check{g}_{i j}$.

(b) As a consequence, the flow exists and is unique on some interval $[0, T)$ with $T>0$.

We stress that this theorem is used only to establish the uniqueness of the Type IIA flow, but not its existence. The reason is that, starting from the flow (2.7) at an initial Type IIA geometry, it is not a priori known whether the flow will remain a Type IIA geometry. Without this information, it is not known whether the above integrability condition (2.11) holds.

\subsection{Formation of singularities and Shi-type estimates}

In general, the fact that a flow may admit short-time existence does not imply that important geometric quantities will evolve by parabolic equations. For example, that is the case for the Ricci flow 29, but it is not the case for the anti-complexified Ricci flow [33]. The Type IIA flow shares common features with both the Ricci flow and the anti-complexified Ricci flow, and one may wonder which way it will behave when it comes to the evolution of the curvature and of the Nijenhuis tensor. It is a very attractive feature of the Type IIA flow that, in this particular respect, it is closer to the Ricci flow. Thus we find

Theorem 6 Consider the source-free Type IIA flow with a smooth, positive, closed, and primitive initial value $\varphi_{0}$.

(a) Then the Nijenhuis tensor evolves by

$$
\begin{aligned}
\left(\partial_{t}-e^{u} \Delta\right)|N|^{2}= & e^{u}\left[-2|\nabla N|^{2}+\left(\nabla^{2} u\right) * N^{2}+R m * N^{2}\right. \\
& \left.+N * \nabla N *(N+\nabla u)+N^{4}+N^{3} * \nabla u+N^{2} *(\nabla u)^{2}\right]
\end{aligned}
$$


(b) The Riemann curvature tensor evolves by

$$
\begin{aligned}
\left(\partial_{t}-e^{u} \Delta\right)|R m|^{2}= & e^{u}\left[-2|\nabla R m|^{2}+\left(\nabla R m+\nabla^{3} u+\nabla^{2} N\right) * \mathcal{O}(R m, \nabla u, N)\right. \\
& \left.+\left(\nabla N * \nabla N+\nabla^{2} u * \nabla^{2} u+1\right) * \mathcal{O}(R m, \nabla u, N)\right] .
\end{aligned}
$$

Here, * denote the bilinear pairings (not to be confused with the Hodge star operator) and $\mathcal{O}(\nabla u, R m, N)$ indicates terms which only depend on $\nabla u, R m$ and $N$.

Other geometric quantities satisfy similar flows, which are written in greater detail in $\S 8$. Using these flows, we can establish the following Shi-type estimates and criteria for extending the flow:

Theorem 7 Assume that we have a solution of the source-free Type IIA flow on some interval $[0, T)$, and that the bound

$$
|u|+|R m| \leq A
$$

holds for some finite constant A. Here Rm denotes the Riemann curvature tensor of the metric $g_{\varphi}$. Then for any multi-index $\alpha$, we have

$$
\left|\nabla^{\alpha} \varphi\right| \leq C(A, \alpha, T, \varphi(0))
$$

for some constant $C(A, \alpha, T, \varphi(0))$. In particular, the Type IIA flow can be continued to an interval $[0, T+\varepsilon)$ for some $\varepsilon>0$.

It may be worth noting that, in this estimate, the estimates for the gradient $|\nabla u|$ are rather special, and make essential use of the underlying Type IIA geometry.

\subsection{The stationary points in the case of no source}

In the case $\rho_{A}=0$, the stationary points of the flow can be identified, once we have developed Type IIA geometry:

Theorem 8 A primitive and closed 3 -form $\varphi$ is a stationary point of the flow if and only if the corresponding almost-complex structure $J_{\varphi}$ is integrable and the norm $|\varphi|$ is constant. Thus $\left(M, J_{\varphi}, \omega\right)$ is then a Kähler manifold, and the metric $g_{\varphi}$ is Kähler and Ricci-flat. 


\subsection{The integrable case and the Calabi conjecture}

The simplest case is that of integrable almost-complex structures, and a complete description of the behavior of the Type IIA flow in this case is provided by the following theorem:

Theorem 9 Assume that the initial value $\varphi_{0}$ of the source-free Type IIA flow is a positive, closed and primitive 3-form, and that the corresponding almost-complex structure $J_{\varphi_{0}}=: J_{0}$ is integrable. Then the source-free Type IIA flow exists for all time, the almost-complex structure $J_{\varphi}$ corresponding to $\varphi$ remains integrable along the flow, and the flow converges in $C^{\infty}$ to a 3-form corresponding to a Kähler Ricci-flat metric.

In fact, the corresponding flow of metrics $g_{\varphi}$ turns out to reduce by diffeomorphisms to the dual Anomaly flow introduced in [20] which applies in all dimensions and gives another proof of the Calabi conjecture. This reduction of the Type IIA flow to the dual Anomaly flow, which was itself motivated by duality considerations for the Type IIB flow, can be viewed as a manifestation of the duality between the Type IIA and the Type IIB theories. We also observe that there are by now several known proofs of the Calabi conjecture, giving each a different sequence converging to the Kähler Ricci-flat metric: besides Yau's original proof, there are for example the proof by the Kähler-Ricci flow [9], by the Anomaly flow [42], by the dual Anomaly flow [20], by the inverse Monge-Ampère flow [10, 7], and by more general parabolic Monge-Ampère flows [43]. Nevertheless, new proofs from an independent geometric set-up remain of considerable interest, as they can detect different types of obstructions. Such a scenario is nicely illustrated in [7].

\section{$2.9 \quad$ Examples}

As we can see from the induced flow of metrics, the Type IIA flow is complicated. However, besides the integrable case which was solved above, there are non-integrable, geometrically interesting cases that can also be worked out completely and which exhibit varied and interesting behaviors. They suggest the possibility of a general phenomenon, namely that in all cases, the Type IIA flow leads to an optimal almost-complex structure with respect to the given symplectic form. A first example is provided by the torus:

Theorem 10 Consider the source-free Type IIA flow on the torus $\mathbf{R}^{6} / \mathbf{Z}^{6}$ with the symplectic form $\omega$ as described in 99.3.1. Consider the Type IIA flow with non-integrable initial data of the form in (9.3.1). Then the flow exists for all time, and $\varphi$ converges as $t \rightarrow \infty$ to a positive harmonic form.

A rich class of models is the special generalized Calabi-Yau (or SGCY) manifolds introduced by de Bartholomeis [12, 13]. These are manifolds of Type IIA geometry with the additional property that $|\varphi|$ is constant. They are also sometimes referred to as symplectic 
half-flat structures. A large subclass is given by nilmanifolds, which are quotients of a nilpotent Lie group by a co-compact lattice, and the other subclass is the solvmanifolds, which are quotients of solvable Lie groups by a co-compact lattice. Details on the models which we consider can be found in $\$ 9.3 .2$, and we shall just state here the main conclusions.

Theorem 11 Consider the source-free Type IIA flow on the nilmanifold and the solvmanifold described in \$9.3.2, with the initial data described there.

(a) In the case of the nilmanifold, with initial data corresponding to (9.29), the flow exists for all time, and the Nijenhuis tensor tends to 0 as $t \rightarrow \infty$.

(b) In the case of the solvmanifold, with initial data corresponding to (9.32), the flow develops a singularity at a finite time $T$. However, the limit of $J_{\varphi}$ as $t \rightarrow T$ exists, and is a harmonic almost-complex structure.

\section{The Type IIA flow as a Laplacian flow}

We start now the proof of the results described in the previous section. For Theorem 1. we do not need any detailed information on the metric $g_{\varphi}$. Rather, we only need the main properties of the corresponding Hermitian connections, and how they differ from the Levi-Civita connection. These have been worked out by Gauduchon [26], and we begin with a brief review of the results from [26] that we require.

\subsection{Gauduchon's formulas for connections on an almost-complex manifold}

We revert momentarily to the general set-up of a smooth manifold $M$ equipped with a Riemannian metric $g$, a compatible almost-complex structure $J$ (not necessarily integrable), and the associated symplectic form $\omega$. This means that $g$ is a positive definite section of the bundle of quadratic forms on $T M, \omega$ is a 2-form on $M, J$ is a section of the bundle of endomorphisms of $T M$ satisfying $J^{2}=-\mathrm{Id}$, and $g(X, Y)=\omega(X, J Y)$ for any two vector fields $X, Y$ on $M$. In local coordinates $x^{j}$, with $X$ and $Y$ given by their components $X^{i}$ and $Y^{j}$, we shall write

$$
g(X, Y)=g_{i j} X^{i} Y^{j}, \quad \omega(X, Y)=\omega_{i j} X^{i} Y^{j}, \quad(J X)^{k}=J_{j}^{k} X^{j} .
$$

In particular $g_{i j}$ and $\omega_{i j}$ are respectively symmetric and anti-symmetric in $i$ and $j$, and the fact that $J$ is a compatible almost-complex structure translates into

$$
J_{q}^{k} J_{j}^{q}=-\delta^{k}, \quad g_{i j}=\omega_{i q} J_{j}^{q}=\omega_{j q} J_{i}^{q} .
$$

Note that $\omega$ is invariant under the action of $J$, i.e. $\omega_{i q} J^{q}{ }_{j}{ }^{i}{ }_{\ell}=\omega_{\ell j}$.

Clearly the structure defined by the triple $(g, \omega, J)$ is determined by any two of its components. Here we do not assume that $\omega$ is closed. For our purposes, we can assume 
that $d \omega$ has no $(3,0)+(0,3)$-components. Obviously this condition is satisfied when $\omega$ is closed, or conformally closed, or when $J$ is integrable. When such a condition is satisfied, $d^{c} \omega=J^{-1} d \omega$ has no $(3,0)+(0,3)$-components either.

Associated to this setup are several important tensors:

- The first is the Nijenhuis tensor $N$, defined as

$$
N(X, Y)=\frac{1}{4}([J X, J Y]-J[J X, Y]-J[X, J Y]-[X, Y]) .
$$

By construction $N$ is a skew-symmetric 2 -form valued in $T M$. Using the metric, we can lower the superscript to the first slot by $N_{X, Y, Z}=g(X, N(Y, Z))$.

- The second is the 3-form $d^{c} \omega$, where by our convention for differential forms, the form $\omega$ is defined from its coefficients $\omega_{i j}$ by

$$
\omega=\frac{1}{2} \omega_{i j} d x^{i} \wedge d x^{j}
$$

The form $d^{c} \omega$ can also be written as $d^{c} \omega=J^{-1} d J \omega=-J d \omega$ since $d^{c}=J^{-1} d J$ and $\omega$ is invariant under the action of $J$. In components, we have

$$
(d \omega)=\frac{1}{3 !}\left(\partial_{i} \omega_{j k}+\partial_{j} \omega_{k i}+\partial_{k} \omega_{i j}\right) d x^{i} \wedge d x^{j} \wedge d x^{k}
$$

and hence

$$
\begin{aligned}
& (d \omega)_{i j k}=\partial_{i} \omega_{j k}+\partial_{j} \omega_{k i}+\partial_{k} \omega_{i j}, \\
& \left(d^{c} \omega\right)_{a b c}=-J_{c}^{k} J_{b}^{j} J_{a}^{i}(d \omega)_{i j k}=-J_{c}^{k} J_{b}^{j} J_{a}^{i}\left(\partial_{i} \omega_{j k}+\partial_{j} \omega_{k i}+\partial_{k} \omega_{i j}\right) .
\end{aligned}
$$

As shown by Gauduchon [26], the construction of Hermitian connections associated to the structure $(g, \omega, J)$ is clearer if we also view $d^{c} \omega$ as a 2 -form valued in $T M$, i.e. a section of $T M \otimes \Lambda^{2} T^{*} M$, in analogy with the Nijenhuis tensor. This can be achieved by raising one index in $d^{c} \omega$, using the metric $g_{i j}$. Unless indicated otherwise, the $T M$-valued 2-form corresponding to $d^{c} \omega$ is obtained by raising the first index, i.e.,

$$
\left(d^{c} \omega\right)^{m}{ }_{j k}=g^{m i}\left(d^{c} \omega\right)_{i j k} .
$$

- The third and the fourth tensors of interest are obtained by decomposing $d^{c} \omega$, viewed as a $T M$-valued 2-form, into components $U$ and $V$ which are respectively odd and even under the following involution $\mathcal{M}$ acting on the space of $T M$-valued 2 -forms,

$$
(\mathcal{M} \Psi)(X, Y)=\Psi(J X, J Y), \quad \Psi \in A^{2}(T M)
$$

where we have denoted the space of $T M$-valued 2-forms by $A^{2}(T M)$. We can then define the $T M$-valued 2-forms $U$ and $V$ by

$$
U=\frac{1}{4}\left(d^{c} \omega+\mathcal{M}\left(d^{c} \omega\right)\right), \quad V=\frac{1}{4}\left(d^{c} \omega-\mathcal{M}\left(d^{c} \omega\right)\right) .
$$


In components,

$$
(\mathcal{M} \Psi)^{m}{ }_{b c}=\Psi^{m}{ }_{j k} J_{c}^{k} J_{b}^{j}
$$

and

$$
U_{b c}^{m}=\frac{1}{4}\left(\left(d^{c} \omega\right)_{b c}^{m}+\left(d^{c} \omega\right)^{m}{ }_{j k} J_{c}^{k} J_{b}^{j}\right), \quad V_{b c}^{m}=\frac{1}{4}\left(\left(d^{c} \omega\right)_{b c}^{m}-\left(d^{c} \omega\right)^{m}{ }_{j k} J_{c}^{k} J_{b}^{j}\right) .
$$

Note that $U_{b c}^{m}$ and $V_{b c}^{m}$ are still anti-symmetric in $b$ and $c$, but if we let $U_{a b c}$ and $V_{a b c}$ the components of the $T^{*} M$-valued 2-form obtained by lowering the index $m$ to an index $a$, then $U_{a b c}$ and $V_{a b c}$ are not anti-symmetric in $a$ and $b$, unlike the form $\left(d^{c} \omega\right)_{a b c}$. The tensors $N, U$, and $V$ satisfy the following Bianchi-type identities

$$
\begin{aligned}
& N_{i j k}+N_{j k i}+N_{k i j}=0, \\
& U_{i j k}+U_{j k i}+U_{k i j}=\left(d^{c} \omega\right)_{i j k}, \quad V_{i j k}+V_{j k i}+V_{k i j}=\frac{1}{2}\left(d^{c} \omega\right)_{i j k} .
\end{aligned}
$$

Given an almost Hermitian structure $(g, \omega, J)$, the Gauduchon line of connections is a line of connections preserving all of $(g, \omega, J)$ which passes through the Chern connection and the projected Levi-Civita connection. If we denote the Levi-Civita connection by $\nabla$, since $d^{c} \omega$ has only type $(2,1)+(1,2)$-components, the Gauduchon line can be parameterized by a real parameter $t$, and the corresponding connection $\mathfrak{D}^{t}$ is given by

$$
\mathfrak{D}_{i}^{t} X^{m}=\nabla_{i} X^{m}+g^{m k}\left(-N_{i j k}-V_{i j k}+t U_{i j k}\right) X^{j} .
$$

Equivalently, if we express a connection $D$ in terms of its connection form $\Gamma(D)^{m}{ }_{i j}$,

$$
D_{i} X^{m}=\partial_{i} X^{m}+\Gamma(D)^{m}{ }_{i j} X^{j}
$$

then we have

$$
\Gamma\left(\mathfrak{D}^{t}\right)^{m}{ }_{i j}=\Gamma(\nabla)^{m}{ }_{i j}+g^{m k}\left(-N_{i j k}-V_{i j k}+t U_{i j k}\right) .
$$

Since the torsion of a connection $D$ is given by

$$
T(D)^{m}{ }_{i j}=\Gamma(D)^{m}{ }_{i j}-\Gamma(D)^{m}{ }_{j i}
$$

and the Levi-Civita connection has zero torsion, it is readily follows that

$$
T\left(\mathfrak{D}^{t}\right)^{m}{ }_{j k}=N^{m}{ }_{j k}+(t-1) U^{m}{ }_{j k}+2 t V^{m}{ }_{j k} .
$$

The two values of $t$ of particular interest to us are:

- $t=0$ : this is the so-called projected Levi-Civita connection (a.k.a. the first canonical connection), which we shall henceforth denote by just $\mathfrak{D}^{0}=\mathfrak{D}$.

- $t=1$ : this is the Chern connection $\nabla^{C}$, also characterized by the condition that $\nabla_{\bar{U}}^{C} V=[\bar{U}, V]^{1,0}$, for any sections $U, V$ of $T^{1,0} M$. Here we have set $\mathbf{C} \otimes T M=T^{1,0} M \oplus$ $T^{0,1} M$ and used $J$ to identify $T M$ with $T^{1,0} M$. The expression $[\bar{U}, V]^{1,0}$ denotes the (1,0)-component of $[\bar{U}, V]$.

The value $t=-1$ gives the Bismut connection, but we shall not need it in this paper. 


\subsubsection{A convenient notation for the action of $J$}

For the convenience of later use, we use the following abbreviations

$$
(J V)^{k}=J_{j}^{k} V^{j}=: V^{J k}, \quad(J W)_{m}=W_{j} J_{m}^{j}=: W_{J m} .
$$

For example, the operator $\mathcal{M}$ acting on a $T M$-valued 2 -form $\Psi$ introduced earlier in (3.4) and (3.6) can now be expressed as

$$
(\mathcal{M} \Psi)^{m}{ }_{b c}=\Psi^{m}{ }_{J b, J c} .
$$

and (3.7) as

$$
U_{b c}^{m}=\frac{1}{4}\left(\left(d^{c} \omega\right)_{b c}^{m}+\left(d^{c} \omega\right)^{m}{ }_{J b, J c}\right), \quad V_{b c}^{m}=\frac{1}{4}\left(\left(d^{c} \omega\right)^{m}{ }_{b c}-\left(d^{c} \omega\right)^{m}{ }_{J b, J c}\right) .
$$

As another example, since $d^{c} \omega$ has no $(0,3)+(3,0)$ component, it satisfies

$$
\left(d^{c} \omega\right)_{J i, j, k}+\left(d^{c} \omega\right)_{i, J j, k}+\left(d^{c} \omega\right)_{i, j, J k}=\left(d^{c} \omega\right)_{J i, J j, J k}
$$

When summing over repeated indices, $J$ can be raised or lowered as follows

$$
X^{k} \alpha_{J k}=X^{k} J_{k}^{l} \alpha_{l}=X^{J k} \alpha_{k}
$$

Moreover, we can insert $J$ in summation indices at the cost of adding a minus sign:

$$
X^{k} \alpha_{k}=-X^{J k} \alpha_{J k}
$$

In this short-hand notation, we have?

$$
\begin{aligned}
\omega_{j k} & =g_{J j, k}=-g_{j, J k}, & g_{j k} & =\omega_{j, J k}=\omega_{k, J j}, \\
\omega^{j k} & =g^{J j, k}=-g^{j, J k}, & g^{j k} & =\omega^{j, J k}=\omega^{k, J j} .
\end{aligned}
$$

\subsubsection{The types of $T M$-valued 2-forms}

All the tensors which we encountered above, namely the Nijenhuis tensor, the tensors $d^{c} \omega$, $U$, and $V$, and the torsion tensors can be viewed as $T M$-valued 2-forms, or equivalently 3-tensors which are antisymmetric in the last two slots. Denote this space by $A^{2}(T M)$ for simplicity. It is convenient to break up elements of $A^{2}(T M)$ into simpler components.

Recall the involution $\mathcal{M}$ on $A^{2}(T M)$ defined by (3.4) or (3.14) in components. Clearly $A^{2}(T M)$ splits into the direct sum of eigenspaces of $\mathcal{M}$ with eigenvalue \pm 1 . We shall

\footnotetext{
${ }^{2}$ Here $\left(\omega^{j k}\right)$ denotes the inverse matrix of $\left(\omega_{j k}\right), \omega^{j k} \omega_{k l}=\delta^{j} l$. It is not the tensor one gets by raising indices using $g_{j k}$. In fact $\omega^{j k}=-g^{j a} \omega_{a b} g^{b k}$.
} 
call the eigenvalue 1 subspace the space of $T M$-valued $(1,1)$-forms, denoted by $A^{1,1}(T M)$. That is, $\Psi \in A^{1,1}(T M)$ if and only if

$$
\Psi_{J j, J k}^{p}=\Psi_{j k}^{p} \text { or equivalently } \Psi_{i, J j, k}+\Psi_{i, j, J k}=0 .
$$

The space of eigenvalue -1 can be decomposed further as follows. We say a $T M$-valued 2 -form $\Psi$ is of type $(2,0)$ or $(0,2)$ if 3

$$
\Psi(J X, Y)=J \Psi(X, Y) \quad \text { or } \quad \Psi(J X, Y)=-J \Psi(X, Y) .
$$

In this way we have a direct sum decomposition

$$
A^{2}(T M)=A^{1,1}(T M) \oplus A^{2,0}(T M) \oplus A^{0,2}(T M) .
$$

In terms of indices we see that

$$
\begin{array}{lcc}
\Psi \in A^{2,0}(T M) & \text { if } \quad \Psi_{J j, k}^{p}=\Psi_{j k}^{J p}=\Psi_{j, J k}^{p} \text { or } \quad \Psi_{J i, j, k}=-\Psi_{i, J j, k}=-\Psi_{i, j, J k}, \\
\Psi \in A^{0,2}(T M) & \text { if } \quad \Psi_{J j, k}^{p}=-\Psi_{j k}^{J p}=\Psi_{j, J k}^{p} \text { or } \quad \Psi_{J i, j, k}=\Psi_{i, J j, k}=\Psi_{i, j, J k} .
\end{array}
$$

Returning to the tensors which we have encountered, their types are as follows:

- It is readily seen that the Nijenhuis tensor is of type $(0,2)$, therefore any contraction of $N$ using either $g$ or $\omega$ yields 0 .

- It is easy to see that the tensor $U^{m}{ }_{j k}$ is of type $(1,1)$. As for $V$, we have by definition

$$
\begin{aligned}
V_{J i, j, k} & =\frac{1}{4}\left(\left(d^{c} \omega\right)_{J i, j, k}-\left(d^{c} \omega\right)_{J i, J j, J k}\right) \stackrel{(3.16)}{=}-\frac{1}{4}\left(\left(d^{c} \omega\right)_{i, J j, k}+\left(d^{c} \omega\right)_{i, j, J k}\right) \\
& =V_{J(J i), J j, k}=-V_{i, J j, k} .
\end{aligned}
$$

and thus $V$ is of type $(2,0)$.

- Finally, tensors such as the torsions of connections on the Gauduchon line and consequently also differences of connections, correspond to forms of mixed types, whose decomposition in $\Lambda^{1,1}(T M) \oplus \Lambda^{2,0}(T M) \oplus \Lambda^{0,2}(T M)$ can be read off from formulas such as (3.11) and (3.12), since we know now the types of $N^{m}{ }_{j k}, U^{m}{ }_{j k}$, and $V^{m}{ }_{j k}$.

\subsection{Proof of Theorem 1}

We can now give the proof of Theorem 1, For simplicity, we shall denote in the subsequent calculations $g_{\varphi}$ and $J_{\varphi}$ by just $g$ and $J$. Recall that the operator $d^{c}$ is defined by $d^{c}=$ $J^{-1} d J$, and that, for a compatible structure $(\omega, g, J)$, we have the identity

$$
d^{\Lambda}:=d \Lambda-\Lambda d=\left(d^{c}\right)^{\dagger}
$$

\footnotetext{
${ }^{3}$ To avoid confusion, we stress that this notion is specific to $A^{2}(T M)$ and is not the same as that of scalar-valued $(2,0)$ or $(0,2)$ forms.
} 
This identity holds even if $J$ is not integrable. Since $\varphi$ is primitive, we can replace in (1.4) $\Lambda d$ by $-d^{\Lambda}$ and rewrite the equation as

$$
\begin{aligned}
\partial_{t} \varphi & =d \Lambda d\left(|\varphi|^{2} \star \varphi\right)-\rho_{A}=-d\left(d^{c}\right)^{\dagger}\left(|\varphi|^{2} \star \varphi\right)-\rho_{A}=-d\left(J^{-1} d J\right)^{\dagger}\left(|\varphi|^{2} \star \varphi\right)-\rho_{A} \\
& =-d J^{-1} d^{\dagger} J\left(|\varphi|^{2} \star \varphi\right)-\rho_{A} .
\end{aligned}
$$

Here we have used the fact that the adjoint $J^{\dagger}$ of $J$ with respect to $g$ is $J^{-1}$, since $J$ is an isometry. We shall see later that $\star \varphi=J \varphi$, so that the above equation can be rewritten as

$$
\partial_{t} \varphi=d J d^{\dagger}\left(|\varphi|^{2} \varphi\right)-\rho_{A}
$$

Thus the theorem would be proved once we can establish the following lemma:

Lemma 1 Let $(M, g, J, \omega)$ be a 6-dimensional compact almost-Hermitian manifold with $d \omega=0$. We have

$$
J d^{\dagger} \varphi=-d^{\dagger} \varphi+2 N^{\dagger} \cdot \varphi
$$

where $N^{\dagger}$ is the operator defined in (2.2).

To establish this, we begin by recalling that the adjoint of the operator $d$ on 3 -forms is given by

$$
\left(d^{\dagger} \varphi\right)_{\alpha \beta}=-\nabla^{\gamma} \varphi_{\gamma \alpha \beta}
$$

where $\nabla$ denotes the Levi-Civita connection of $g_{i j}$, which has no torsion. We need to apply the operator $J$ to both sides of this equation. For this, we need in turn the following lemma:

\section{Lemma 2}

$$
\nabla_{k} J_{b}^{a}=-2 N_{J k}{ }_{b}^{a} .
$$

The point of this lemma is that the Levi-Civita connection does not necessarily respect the almost-complex structure $J$. However, we can write it in terms of the projected LeviCivita connection $\mathfrak{D}$ which does, at the cost of having to handle in addition terms coming from difference of connections, which gives us the Nijenhuis tensor. This lemma follows directly from (3.11), where $U=V=0$ as $\omega$ is closed.

Returning to the proof of Lemma 1, since $\varphi_{\alpha, J \beta, J \gamma}=-\varphi_{\alpha \beta \gamma}$ by Lemma 2 and [6, we find

$$
\begin{aligned}
\left(J d^{\dagger} \varphi\right)_{k j} & =-J^{\alpha}{ }_{k} J^{\beta}{ }_{j} \nabla^{\gamma} \varphi_{\gamma \alpha \beta} \\
& =-\nabla^{\gamma}\left(J_{k}^{\alpha} J^{\beta}{ }_{j} \varphi_{\gamma \alpha \beta}\right)-2\left(N_{J \gamma}{ }_{k} J^{\beta}{ }_{j}+N_{J \gamma}{ }_{j}{ }_{j} J_{k}{ }_{k}\right) \varphi^{\gamma}{ }_{\alpha \beta} \\
& =-\nabla^{\gamma} \varphi_{\gamma, J k, J j}-2\left(N^{\lambda \mu}{ }_{k} \varphi_{\mu j \lambda}-N^{\lambda \mu}{ }_{j} \varphi_{\mu k \lambda}\right) \\
& =-\left(d^{\dagger} \varphi\right)_{k j}+2\left(N^{\dagger} \cdot \varphi\right)_{k j} .
\end{aligned}
$$

This completes the proof of Lemma 1, Replacing $\varphi$ by $|\varphi|^{2} \varphi$ in Lemma 1, we also obtain Theorem 1. 


\section{The principal symbol of the Type IIA equation}

Our next task is to identify the symbol and the eigenvalues of the Type IIA equation.

\subsection{Almost-complex structures and 3-forms}

For this and for the rest of the paper, we make essential use of Hitchin's construction of an almost-complex structure $J_{\varphi}$ and a metric $g_{\varphi}$ from a 3 -form $\varphi$ on a 6 -dimensional manifold [30]. We begin by recalling the results that we need.

\subsubsection{Hitchin's construction}

Let $V$ be a 6-dimensional oriented vector space over $\mathbf{R}$. Following Hitchin [30], for any 3-form $\varphi \in \Lambda^{3} V^{*}$, one can define a linear map $K_{\varphi}: V \rightarrow \Lambda^{5} V^{*} \cong V \otimes \Lambda^{6} V^{*}$ by

$$
K_{\varphi}(v)=-\iota_{v} \varphi \wedge \varphi=-e_{i} \otimes e^{i} \wedge \iota_{v} \varphi \wedge \varphi
$$

where $\left\{e_{i}\right\}$ is an arbitrary basis of $V$ and $\left\{e^{i}\right\}$ its dual basis in $V^{*}$. It follows that

$$
\lambda_{\varphi}:=\frac{1}{6} \operatorname{tr}_{V} K_{\varphi}^{2} \in\left(\Lambda^{6} V^{*}\right)^{2}
$$

is well-defined and it makes sense to talk about the sign of $\lambda_{\varphi}$. In general $\lambda_{\varphi}$ is a homogeneous degree 4 polynomial in the components of $\varphi$. When $\lambda_{\varphi}<0$, as $V$ is oriented, one can take $\sqrt{-\lambda_{\varphi}} \in \Lambda^{6} V^{*}$ to be the positive square root of $-\lambda_{\varphi}$. It is proved in [30]

$$
J_{\varphi}:=\frac{K_{\varphi}}{\sqrt{-\lambda_{\varphi}}}: V \rightarrow V
$$

defines a complex structure on $V$. Note that $\lambda_{\varphi}<0$ is an open condition. In fact, the set $\left\{\varphi \in \Lambda^{3} V^{*}: \lambda_{\varphi}<0\right\}$ forms an open orbit in $\Lambda^{3} V^{*}$ of the natural $G L(V)$-action. Furthermore, there is a basis $\left\{e^{i}\right\}$ of $V^{*}$ where $\varphi$ takes the following "canonical form",

$$
\varphi=\operatorname{Re}\left(e^{1}+i e^{2}\right) \wedge\left(e^{3}+i e^{4}\right) \wedge\left(e^{5}+i e^{6}\right)=e^{135}-e^{146}-e^{245}-e^{236}
$$

and $e^{123456}$ defines a positive volume form. In this basis, one can easily check that $J_{\varphi} e_{2 k-1}=$ $e_{2 k}$ and $J_{\varphi} e_{2 k}=-e_{2 k-1}$ for $k=1,2,3$. Therefore $\varphi$ is the real part of a $(3,0)$-form with respect to the complex structure $J_{\varphi}$. It follows that the imaginary part

$$
\hat{\varphi}=\operatorname{Im}\left(e^{1}+i e^{2}\right) \wedge\left(e^{3}+i e^{4}\right) \wedge\left(e^{5}+i e^{6}\right)=e^{136}+e^{145}+e^{235}-e^{246}
$$

is also determined by $\varphi$ through $\hat{\varphi}=J_{\varphi} \varphi$, meaning that

$$
\hat{\varphi}(X, Y, Z)=\varphi\left(J_{\varphi} X, J_{\varphi} Y, J_{\varphi} Z\right)
$$

for any $X, Y, Z \in V$. Furthermore two forms $\varphi$ and $\tilde{\varphi}$ define the same complex structure $J_{\varphi}$ if and only if they are related by $\mathbf{C}^{*}$-action

$$
\tilde{\varphi}=\rho \cdot \operatorname{Re}\left(e^{-i \theta}(\varphi+i \hat{\varphi})\right) .
$$

\footnotetext{
${ }^{4}$ Here we adopt the convention used in [15, 16].
} 


\subsection{2 $J_{\varphi}$ and symplectic structures}

Now let us assume that $V$ is equipped with a symplectic form $\omega \in \Lambda^{2} V^{*}$ so $V$ is canonically oriented by $\omega^{3} / 3$ !. A natural question is when the symplectic form $\omega$ is invariant under the induced complex structure $J_{\varphi}$. The answer is very simple:

Lemma $3 \omega$ is $J_{\varphi}$-invariant if and only if $\varphi$ is primitive in the sense that $\omega \wedge \varphi=0$.

Proof. As stated above, we can choose a basis such that $\varphi=e^{135}-e^{146}-e^{245}-e^{236}$. In this coordinate, we may write $\omega=a_{i j} e^{i} \wedge e^{j}$ with $a_{i j}=-a_{j i}$. The condition that $\omega\left(e_{i}, e_{j}\right)=\omega\left(J_{\varphi} e_{i}, J_{\varphi} e_{j}\right)$ for any $i$ and $j$ is equivalent to the following system of linear equations

$$
\begin{array}{ll}
a_{13}=a_{24}, & a_{14}=-a_{23}, \\
a_{16}=-a_{25}, & a_{15}=a_{26}, \\
a_{35}, & a_{36}=-a_{45} .
\end{array}
$$

These are exactly the equations for $\varphi$ being primitive in the sense that $\omega \wedge \varphi=0$. Q.E.D.

For primitive $\varphi$, we can consider then the Hermitian form $g_{\varphi}(X, Y)=\omega\left(X, J_{\varphi} Y\right)$. We shall say that $\varphi$ is positive if $g_{\varphi}$ is positive, in which case $g_{\varphi}$ is a metric, and the triple $\left(\omega, J_{\varphi}, g_{\varphi}\right)$ is compatible 5. The positivity of $\varphi$ is an open condition. Once we have a Riemannian metric and an orientation, we have the associated Hodge star operator $\star$. It is straightforward to check that

$$
\star \varphi=\hat{\varphi}, \quad \star \hat{\varphi}=-\varphi .
$$

In particular we know that $\hat{\varphi}$ is primitive if $\varphi$ is primitive.

Altogether, assuming the presence of $\omega$ and that $\varphi$ is primitive and positive, we may upgrade the previous choice of orthonormal basis $\left\{e_{j}\right\}_{j=1}^{6}$ to the following useful statement:

Lemma 4 (Normal form of $\varphi$ )

There exists an orthonormal basis $\left\{e_{j}\right\}_{j=1}^{6}$ of $V$ (with respect to $g$ ) such that

$$
\begin{aligned}
& \omega=e^{1} \wedge e^{2}+e^{3} \wedge e^{4}+e^{5} \wedge e^{6} \\
& \varphi=M \operatorname{Re}\left(e^{1}+i e^{2}\right) \wedge\left(e^{3}+i e^{4}\right) \wedge\left(e^{5}+i e^{6}\right),
\end{aligned}
$$

where $M=\frac{1}{2}|\varphi|>0$. It follows that

$$
\sqrt{-\lambda_{\varphi}}=\frac{1}{2}|\varphi|^{2} \frac{\omega^{3}}{3 !}
$$

\footnotetext{
${ }^{5}$ This notion of positivity is different from the one defined in [30], which does not involve a symplectic form.
} 
Using this upgraded version of canonical form of $\varphi$, one can check that the following key formula for the metric $g_{\varphi}$ holds:

Lemma 5 In any coordinate system, we can write

$$
\left(g_{\varphi}\right)_{i j}=-|\varphi|^{-2} \varphi_{i a b} \varphi_{j c d} \omega^{a c} \omega^{b d}=2|\varphi|^{-2} \frac{\iota_{i} \varphi \wedge \iota_{j} \varphi \wedge \omega}{\omega^{3} / 3 !}
$$

The metric $\tilde{g}_{\varphi}$ introduced in (2.3) is then given by

$$
\left(\tilde{g}_{\varphi}\right)_{i j}=-\varphi_{i a b} \varphi_{j c d} \omega^{a c} \omega^{b d}
$$

Clearly $\tilde{g}_{\varphi}$ is conformal to $g_{\varphi}$ and its associated Kähler form is $\tilde{\omega}_{\varphi}=|\varphi|^{2} \omega$. Since $|\varphi|^{2}$ is the square root of a complicated homogeneous degree 4 polynomial in components of $\varphi$, the metric $\tilde{g}_{\varphi}$ has the advantage that its expression is algebraic in $\varphi$, which makes it much easier to compute with. Also, the volume form of $g_{\varphi}$ is just $\omega^{3} / 3$ !, but we can recapture $|\varphi|^{2}$ from the volume form of $\tilde{g}_{\varphi}$.

\subsubsection{Basic identities}

As always we fix a symplectic form $\omega$. Let $\varphi$ be a positive primitive 3 -form so that it defines an almost complex structure $J_{\varphi}$ compatible with $\omega$. For simplicity, we shall denote in the subsequent calculations $g_{\varphi}$ and $J_{\varphi}$ by just $g$ and $J$. Furthermore we can define another primitive 3 -form $\hat{\varphi}=J \varphi=\star \varphi$ such that $\Omega=\varphi+i \hat{\varphi}$ is a nowhere vanishing $(3,0)$-form with respect to $J$, i.e. a complex volume form that trivializes the canonical bundle of $(M, J)$.

Lemma 6 The 3 -forms $\varphi$ and $\hat{\varphi}$ are related to each other by

$$
\begin{aligned}
& \varphi_{i j k}=\hat{\varphi}_{J i, j, k}=\hat{\varphi}_{i, J j, k}=\hat{\varphi}_{i, j, J k}=-\varphi_{J i, J j, k}=-\varphi_{J i, j, J k}=-\varphi_{i, J j, J k}=-\hat{\varphi}_{J i, J j, J k} \\
& \hat{\varphi}_{i j k}=-\varphi_{J i, j, k}=-\varphi_{i, J j, k}=-\varphi_{i, j, J k}=-\hat{\varphi}_{J i, J j, k}=-\hat{\varphi}_{J i, j, J k}=-\hat{\varphi}_{i, J j, J k}=\varphi_{J i, J j, J k} .
\end{aligned}
$$

Proof. Since $\varphi+i \hat{\varphi}$ is of type $(3,0)$, we have $\iota_{\partial_{k}+i J \partial_{k}}(\varphi+i \hat{\varphi})=0$. By taking the real and imaginary parts of the above equation and its iterations gives the desired identities. Q.E.D.

Since $\varphi$ and $\hat{\varphi}$ are type $(3,0)+(0,3)$-forms, for any 1 -form $\mu$, we know that both $\mu \wedge \varphi$ and $\mu \wedge \hat{\varphi}$ are of type $(3,1)+(1,3)$, so

$$
\mu \wedge \varphi=-J(\mu \wedge \varphi)=-J \mu \wedge \hat{\varphi}
$$

It is not hard to verify that wedging with $\varphi$ or $\hat{\varphi}$ gives an isomorphism from the space of real 1 -forms to the space of real $(3,1)+(1,3)$-forms. Note that the primitiveness of $\varphi$ with 
respect to $\omega$ implies the primitiveness of $\varphi$ with respect to $\tilde{\omega}$, and hence $\tilde{\omega}^{j i} \varphi_{i j k}=0$, or equivalently,

$$
\begin{aligned}
& \tilde{\omega}_{i j} \varphi_{k l m}-\tilde{\omega}_{k j} \varphi_{i l m}-\tilde{\omega}_{l j} \varphi_{k i m}-\tilde{\omega}_{m j} \varphi_{k l i}-\tilde{\omega}_{i k} \varphi_{j l m} \\
& -\tilde{\omega}_{i l} \varphi_{k j m}-\tilde{\omega}_{i m} \varphi_{k l j}+\tilde{\omega}_{k l} \varphi_{i j m}+\tilde{\omega}_{m l} \varphi_{k j i}+\tilde{\omega}_{m k} \varphi_{j l i}=0 .
\end{aligned}
$$

We also have the following simple lemma:

Lemma 7 The following are equivalent:

(a) $d \hat{\varphi}=0$;

(b) The almost-complex structure $J$ is integrable.

In both cases, the $(3,0)$-form $\Omega$ is holomorphic and the form $\varphi$ is harmonic.

Proof. If $J$ is integrable, then $\partial \Omega=0$ since it is a $(4,0)$-form in a 3-dimensional complex manifold. Similarly for $\bar{\partial} \bar{\Omega}$. But $\varphi=\frac{1}{2}(\Omega+\bar{\Omega})$, so $d \varphi=0$ implies $0=\bar{\partial} \Omega+\partial \bar{\Omega}$, and hence $\Omega$ is holomorphic and $d \Omega=0$. This implies that $d \hat{\varphi}=0$.

Conversely, if $d \hat{\varphi}=0$, then we know that $\Omega=\varphi+\sqrt{-1} \hat{\varphi}$ is also closed. Therefore for any $(1,0)$-form $\lambda$, we know $\lambda \wedge \Omega=0$, hence

$$
0=d(\lambda \wedge \Omega)=d \lambda \wedge \Omega
$$

so we deduce that $d \lambda$ has no $(0,2)$-components, which implies that $J$ is integrable by Frobenius theorem. Q.E.D.

The defining equation for $\tilde{g}$ tells us the effect of contracting twice with $\omega^{i j}$ a quadratic polynomial in $\varphi$. It actually follows from a stronger identity with only one contraction, and which can be verified explicitly using the normal form of $\varphi$ in Lemma 4. Using the fact that $g^{i j}=\omega^{i, J j}$, we can readily deduce the effect of contracting with $g^{i j}$. We summarize these contractions in the following lemma:

Lemma 8 The following quadratic identities hold:

$$
\begin{aligned}
\omega^{i j} \varphi_{i a b} \varphi_{j c d} & =\frac{1}{4}\left(\omega_{a c} \tilde{g}_{b d}-\omega_{b c} \tilde{g}_{a d}-\omega_{a d} \tilde{g}_{b c}+\omega_{b d} \tilde{g}_{a c}\right) \\
g^{i j} \varphi_{i a b} \varphi_{j c d} & =\frac{1}{4}\left(g_{a c} \tilde{g}_{b d}-g_{b c} \tilde{g}_{a d}+\omega_{a d} \tilde{\omega}_{b c}-\omega_{b d} \tilde{\omega}_{a c}\right) \\
g^{a c} g^{b d} \varphi_{i a b} \varphi_{j c d} & =\tilde{g}_{i j} .
\end{aligned}
$$

\subsection{The variation $\delta(\hat{\varphi})$}

The key variational formula for $\hat{\varphi}$ is given by the following lemma: 


\section{Lemma 9}

$$
\delta(\hat{\varphi})=-J_{\varphi}(\delta \varphi)+2 \frac{\delta \varphi \wedge \varphi}{\varphi \wedge \hat{\varphi}} \varphi+2 \frac{\delta \varphi \wedge \hat{\varphi}}{\varphi \wedge \hat{\varphi}}
$$

When $\varphi$ is primitive and positive with respect to a symplectic form $\omega$, the above formula can be written as

$$
\delta(\hat{\varphi})=-J_{\varphi}(\delta \varphi)-\frac{2(\delta \varphi, \hat{\varphi})}{|\varphi|^{2}} \varphi+\frac{2(\delta \varphi, \varphi)}{|\varphi|^{2}} \hat{\varphi} .
$$

Proof. This is a purely linear algebra problem. We break its proof into two steps.

- Step 1: The primitive case.

Choose a nondegenerate $(1,1)$-form $\omega$ compatible with $J_{\varphi}$. We shall first prove Lemma 9 under the assumption that $\delta \varphi$ is primitive with respect to $\omega$. By Lemma 4, one can find an orthonormal basis $\left\{e^{i}\right\}_{i=1}^{6}$ of $V^{*}$ such that

$$
\begin{aligned}
\omega & =e^{1} \wedge e^{2}+e^{3} \wedge e^{4}+e^{5} \wedge e^{6} \\
\varphi & =M\left(e^{1} \wedge e^{3} \wedge e^{5}-e^{1} \wedge e^{4} \wedge e^{6}-e^{2} \wedge e^{4} \wedge e^{5}-e^{2} \wedge e^{3} \wedge e^{6}\right), \\
\star \varphi & =M\left(e^{1} \wedge e^{3} \wedge e^{6}+e^{1} \wedge e^{4} \wedge e^{5}+e^{2} \wedge e^{3} \wedge e^{5}-e^{2} \wedge e^{4} \wedge e^{6}\right),
\end{aligned}
$$

where $M=\frac{1}{2}|\varphi|>0$. For simplicity, we denote $e^{123456}=\omega^{3} / 3$ ! by $\epsilon$. Let $\delta \varphi=\mu=$ $\frac{1}{3 !} \mu_{i j k} e^{i} \wedge e^{j} \wedge e^{k}$. Straightforward computation gives us

$\delta\left(K_{\varphi}\right)=2 M \epsilon\left[\begin{array}{cccccc}A_{1} & \mu_{135}-\mu_{146} & -\mu_{125} & \mu_{126} & \mu_{123} & -\mu_{124} \\ \mu_{236}+\mu_{245} & -A_{1} & \mu_{126} & \mu_{125} & -\mu_{124} & -\mu_{123} \\ \mu_{345} & -\mu_{346} & A_{2} & \mu_{135}-\mu_{236} & -\mu_{134} & \mu_{234} \\ -\mu_{346} & -\mu_{345} & \mu_{245}+\mu_{146} & -A_{2} & \mu_{234} & \mu_{134} \\ -\mu_{356} & \mu_{456} & \mu_{156} & -\mu_{256} & A_{3} & \mu_{135}-\mu_{245} \\ \mu_{456} & \mu_{356} & -\mu_{256} & -\mu_{156} & \mu_{236}+\mu_{146} & -A_{3}\end{array}\right]$,

where

$$
\begin{aligned}
& A_{1}=\frac{\mu_{246}+\mu_{136}+\mu_{145}-\mu_{235}}{2}, \\
& A_{2}=\frac{\mu_{246}+\mu_{136}-\mu_{145}+\mu_{235}}{2}, \\
& A_{3}=\frac{\mu_{246}-\mu_{136}+\mu_{145}+\mu_{235}}{2} .
\end{aligned}
$$

It follows that

$$
\delta\left(K_{\varphi}^{2}\right)=4 M^{3} \epsilon^{2}\left(\mu_{236}+\mu_{245}-\mu_{135}+\mu_{146}\right) \operatorname{Id}_{V}=4 M^{2} \epsilon^{2} \frac{\hat{\varphi} \wedge \mu}{\epsilon} \operatorname{Id}_{V}
$$


Therefore

$$
\begin{aligned}
\delta\left(-\lambda_{\varphi}\right) & =4 M^{2} \epsilon^{2} \frac{\mu \wedge \hat{\varphi}}{\epsilon}=4 M^{2} \epsilon^{2}(\mu, \varphi) \\
\delta \sqrt{-\lambda_{\varphi}} & =\frac{1}{2} \delta\left(|\varphi|^{2}\right) \epsilon=\mu \wedge \hat{\varphi}=(\delta \varphi, \varphi) \epsilon
\end{aligned}
$$

which agrees with Hitchin's formula [30, Proposition 4]. As a consequence, we have

$$
\delta\left(J_{\varphi}\right)=\frac{\delta\left(K_{\varphi}\right)}{2 M^{2} \epsilon}-\frac{\mu \wedge \hat{\varphi}}{2 M^{2} \epsilon} J_{\varphi}
$$

For simplicity of notation, we introduce the following

$$
\begin{aligned}
F & :=\frac{\delta\left(K_{\varphi}\right)}{2 M \epsilon} \\
\varphi_{0} & :=e^{1} \wedge e^{3} \wedge e^{5}-e^{1} \wedge e^{4} \wedge e^{6}-e^{2} \wedge e^{4} \wedge e^{5}-e^{2} \wedge e^{3} \wedge e^{6}, \\
\hat{\varphi}_{0} & :=e^{2} \wedge e^{3} \wedge e^{6}+e^{1} \wedge e^{4} \wedge e^{5}+e^{2} \wedge e^{3} \wedge e^{5}-e^{2} \wedge e^{4} \wedge e^{6} \\
d z^{k} & :=e^{2 k-1}+i e^{2 k}, k=1,2,3 .
\end{aligned}
$$

Again by straightforward calculation, we get

$$
\begin{aligned}
& F \cdot d z^{1}=i B_{0} d z^{1}+\left(A_{1}+i B_{1}\right) d \bar{z}^{1}+C_{3} d \bar{z}^{2}+C_{2} d \bar{z}^{3} \\
& F . d z^{2}=C_{3} d \bar{z}^{1}+i B_{0} d z^{2}+\left(A_{2}+i B_{2}\right) d \bar{z}^{2}+C_{1} d \bar{z}^{3} \\
& F . d z^{3}=C_{2} d \bar{z}^{1}+C_{1} d \bar{z}^{2}+i B_{0} d z^{3}+\left(A_{3}+i B_{3}\right) d \bar{z}^{3} .
\end{aligned}
$$

where

$$
\begin{aligned}
B_{0} & =\frac{1}{2}\left(\mu_{135}-\mu_{146}-\mu_{236}-\mu_{245}\right)=\frac{\mu \wedge \hat{\varphi}_{0}}{2 \epsilon} \\
B_{1} & =\frac{1}{2}\left(\mu_{135}-\mu_{146}+\mu_{236}+\mu_{245}\right) \\
B_{2} & =\frac{1}{2}\left(\mu_{135}+\mu_{146}-\mu_{236}+\mu_{245}\right) \\
B_{3} & =\frac{1}{2}\left(\mu_{135}+\mu_{146}+\mu_{236}-\mu_{245}\right) \\
C_{1} & =\mu_{156}-i \mu_{256}=-\mu_{134}+i \mu_{234} \\
C_{2} & =-\mu_{356}+i \mu_{456}=\mu_{123}-i \mu_{124} \\
C_{3} & =\mu_{345}-i \mu_{346}=-\mu_{125}+i \mu_{126}
\end{aligned}
$$

Here to obtain expressions of $C_{j}$ one makes use of primitiveness of $\mu$. For completeness we also introduce

$$
A_{0}=\frac{\mu_{246}-\mu_{235}-\mu_{136}-\mu_{145}}{2}=\frac{\mu \wedge \varphi_{0}}{2 \epsilon} .
$$


Collecting all these together, we get

$$
\begin{aligned}
F .\left(\varphi_{0}+i \hat{\varphi}_{0}\right)= & F \cdot\left(d z^{1} \wedge d z^{2} \wedge d z^{3}\right) \\
= & F \cdot\left(d z^{1}\right) \wedge d z^{2} \wedge d z^{3}+d z^{1} \wedge F .\left(d z^{2}\right) \wedge d z^{3}+d z^{1} \wedge d z^{2} \wedge F .\left(d z^{3}\right) \\
= & 3 i B_{0} d z^{1} \wedge d z^{2} \wedge d z^{3}+\left(A_{1}+i B_{1}\right) d \bar{z}^{1} \wedge d z^{2} \wedge d z^{3} \\
& +\left(A_{2}+i B_{2}\right) d z^{1} \wedge d \bar{z}^{2} \wedge d z^{3}+\left(A_{3}+i B_{3}\right) d z^{1} \wedge d z^{2} \wedge d \bar{z}^{3} \\
& +C_{1} d z^{1} \wedge\left(d \bar{z}^{3} \wedge d z^{3}+d z^{2} \wedge d \bar{z}^{2}\right)-C_{2} d z^{2} \wedge\left(d \bar{z}^{3} \wedge d z^{3}+d z^{1} \wedge d \bar{z}^{1}\right) \\
& +C_{3} d z^{3} \wedge\left(d z^{1} \wedge d \bar{z}^{1}+d \bar{z}^{2} \wedge d z^{2}\right) .
\end{aligned}
$$

Notice we can express $\mu$ as

$$
\begin{aligned}
\mu= & -\frac{i}{4}\left[\left(C_{1} d z^{1}+\overline{C_{1}} d \bar{z}^{1}\right) \wedge\left(d \bar{z}^{3} \wedge d z^{3}+d z^{2} \wedge d \bar{z}^{2}\right)\right. \\
& -\left(C_{2} d z^{2}+\overline{C_{2}} d \bar{z}^{2}\right) \wedge\left(d \bar{z}^{3} \wedge d z^{3}+d z^{1} \wedge d \bar{z}^{1}\right) \\
& \left.+\left(C_{3} d z^{3}+\overline{C_{3}} d \bar{z}^{3}\right) \wedge\left(d z^{1} \wedge d \bar{z}^{1}+d \bar{z}^{2} \wedge d z^{2}\right)\right]+\frac{B_{0}}{2} \varphi_{0}-\frac{A_{0}}{2} \hat{\varphi}_{0} \\
& -\frac{i}{4}\left(A_{1}+i B_{1}\right) d \bar{z}^{1} \wedge d z^{2} \wedge d z^{3}+\frac{i}{4}\left(A_{1}-i B_{1}\right) d z^{1} \wedge d \bar{z}^{2} \wedge d \bar{z}^{3} \\
& -\frac{i}{4}\left(A_{2}+i B_{2}\right) d z^{1} \wedge d \bar{z}^{2} \wedge d z^{3}+\frac{i}{4}\left(A_{2}-i B_{2}\right) d \bar{z}^{1} \wedge d z^{2} \wedge d \bar{z}^{3} \\
& -\frac{i}{4}\left(A_{3}+i B_{3}\right) d z^{1} \wedge d z^{2} \wedge d \bar{z}^{3}+\frac{i}{4}\left(A_{3}-i B_{3}\right) d \bar{z}^{1} \wedge d \bar{z}^{2} \wedge d z^{3} .
\end{aligned}
$$

By taking real part of (4.23) we get

$$
F . \varphi_{0}=2 J_{\varphi} \mu-A_{0} \varphi_{0}-4 B_{0} \hat{\varphi}_{0} .
$$

Since

$$
\begin{aligned}
J_{\varphi} \cdot \varphi_{0} & =\varphi_{0}\left(J_{\varphi} \cdot, \cdot, \cdot\right)+\varphi_{0}\left(\cdot, J_{\varphi} \cdot, \cdot\right)+\varphi_{0}\left(\cdot, \cdot, J_{\varphi} \cdot\right)=-3 \hat{\varphi}_{0} \\
\delta\left(J_{\varphi}\right) & =\frac{1}{M}\left(F-B_{0} J_{\varphi}\right)
\end{aligned}
$$

it follows that

$$
\delta\left(J_{\varphi}\right) \cdot \varphi_{0}=\frac{1}{M}\left(2 J_{\varphi} \mu-A_{0} \varphi_{0}-B_{0} \hat{\varphi}_{0}\right) .
$$

Consequently we obtain

$$
\delta \hat{\varphi}=J_{\varphi} \delta \varphi-\delta\left(J_{\varphi}\right) \cdot \varphi=-J_{\varphi} \mu+A_{0} \varphi_{0}+B_{0} \hat{\varphi}_{0} .
$$

Rewrite this equation in a coordinate-free manner, we obtain the desired formula.

- Step 2: The general case. 
Choosing $\omega$ as before, a general variation $\delta \varphi$ takes the form

$$
\delta \varphi=\mu+\omega \wedge \lambda
$$

where $\mu$ is primitive with respect to $\omega$ and $\lambda$ is a 1-form. By linearity, we only need to prove our formula for $\delta \varphi=\omega \wedge \lambda$. By symmetry, we may assume that $\lambda=N e^{1}$ for some number $N$. Therefore $\omega \wedge \lambda=N e^{1} \wedge\left(e^{34}+e^{56}\right)$ is a linear combination of $e^{1} \wedge\left(e^{34}-e^{56}\right)$ and $e^{1} \wedge\left(K e^{34}-e^{56}\right)$ for some constant $K>1$. Notice that $e^{1} \wedge\left(e^{34}-e^{56}\right)$ is primitive with respect to $\omega$, and $e^{1} \wedge\left(K e^{34}-e^{56}\right)$ is primitive with respect to another $J_{\varphi}$-compatible $(1,1)$-form $\omega^{\prime}=e^{12}+K e^{34}+e^{56}$. By linearity we reduce Step 2 to Step 1 with $\omega$ replaced by $\omega^{\prime}$. Q.E.D.

\subsection{The eigenvalues of the principal symbol}

With all preparations from last section, we are now ready to compute the principal symbol of the Type IIA flow (1.4) without source. When $\varphi$ is primitive, the right hand side of the Type IIA flow is also primitive, so we only need to consider primitive variations in Lemma 9. which takes the form

$$
\delta\left(|\varphi|^{2} \hat{\varphi}\right)=-|\varphi|^{2} J(\delta \varphi)-2(\delta \varphi, \hat{\varphi}) \varphi+4(\delta \varphi, \varphi) \hat{\varphi}
$$

Thus the symbol of the leading term in the Type IIA flow is given by

$$
\delta \varphi \mapsto \xi \wedge \Lambda\left\{\xi \wedge\left(-|\varphi|^{2} J(\delta \varphi)-2(\delta \varphi, \hat{\varphi}) \varphi+4(\delta \varphi, \varphi) \hat{\varphi}\right)\right\}
$$

and whether the flow is parabolic or not, depends on the eigenvalues of this operator. Since by our assumption, the right hand side of the flow is primitive and admits an integrability operator $d$. Thus by the Hamilton-Nash-Moser theorem [29], we can restrict $\delta \varphi$ to the space

$$
W=\left\{\delta \varphi \in \Lambda^{3} V^{*}: \xi \wedge \delta \varphi=0, \Lambda(\delta \varphi)=0\right\}
$$

As before, we may choose an orthonormal basis $\left\{e^{i}\right\}_{i=1}^{6}$ of $V^{*}$ such that

$$
\begin{aligned}
\omega & =e^{1} \wedge e^{2}+e^{3} \wedge e^{4}+e^{5} \wedge e^{6} \\
\varphi & =M\left(e^{1} \wedge e^{3} \wedge e^{5}-e^{1} \wedge e^{4} \wedge e^{6}-e^{2} \wedge e^{4} \wedge e^{5}-e^{2} \wedge e^{3} \wedge e^{6}\right), \\
\star \varphi & =M\left(e^{1} \wedge e^{3} \wedge e^{6}+e^{1} \wedge e^{4} \wedge e^{5}+e^{2} \wedge e^{3} \wedge e^{5}-e^{2} \wedge e^{4} \wedge e^{6}\right) .
\end{aligned}
$$

As we only care about the sign of the eigenvalues of the principal symbol, we may assume that $|\xi|=2 M=1$. Moreover by rotational symmetry we may assume that $\xi=e^{1}$. Under such reduction, it is easy to see that

$$
W=\left\{e^{1} \wedge \gamma: \gamma \in \Lambda^{2}\left(V^{\prime}\right)^{*}, \Lambda^{\prime} \gamma=0\right\}
$$


where $V^{\prime}=\operatorname{span}\left\{e_{j}\right\}_{j=3}^{6}$ equipped with the symplectic form $\omega^{\prime}=e^{3} \wedge e^{4}+e^{5} \wedge e^{6}$ and $\Lambda^{\prime}$ is the contraction with respect to $\omega^{\prime}$. In this way we can also simplify the operator to

$$
e^{1} \wedge \gamma \mapsto e^{1} \wedge\left[J \gamma+\frac{1}{2}\left(\gamma, e^{3} \wedge e^{6}+e^{4} \wedge e^{5}\right)\left(e^{3} \wedge e^{6}+e^{4} \wedge e^{5}\right)+\left(\gamma, e^{35}-e^{46}\right)\left(e^{35}-e^{46}\right)\right]
$$

which is equivalent to

$$
\gamma \mapsto J \gamma+\frac{1}{2}\left(\gamma, e^{3} \wedge e^{6}+e^{4} \wedge e^{5}\right)\left(e^{3} \wedge e^{6}+e^{4} \wedge e^{5}\right)+\left(\gamma, e^{35}-e^{46}\right)\left(e^{35}-e^{46}\right) .
$$

Then it is clear that the eigenvalues are $\lambda=1$ (multiplicity 4) with eigenvectors $\gamma=$ $e^{3} \wedge e^{4}-e^{5} \wedge e^{6}, e^{3} \wedge e^{5}+e^{4} \wedge e^{6}, e^{3} \wedge e^{6}-e^{4} \wedge e^{5}$ and $e^{3} \wedge e^{5}-e^{4} \wedge e^{6}, \lambda=0$ with eigenvector $\gamma=e^{3} \wedge e^{6}+e^{4} \wedge e^{5}$. We summarize our findings in the following lemma:

Lemma 10 The leading symbol in the Type IIA flow, restricted to closed and primitive forms, is only weakly parabolic. More precisely, it has an eigenvalue $\lambda=1$ with multiplicity 4, and an eigenvalue $\lambda=0$ with multiplicity 1.

\section{$5 \quad$ Proof of Theorem 2: existence}

In this section we establish the short-time existence of the Type IIA flow. As we saw in $\S 4$, the flow is not strictly parabolic, and the presence of the symplectic form prevents a direct application of either the reparametrization arguments of [14] or the Hamilton-Nash-Moser theorem of [29]. Rather, we proceed as follows: first we do apply a reparametrization, but we have to accompany it at the same time with a flow of the symplectic form. This new coupled flow of $(\varphi, \omega)$ is still not strictly parabolic, but one of its key properties is that it admits a strictly regularization with integrability condition, to which the Hamilton-NashMoser theorem can apply. Thus we obtain the short-time existence for a regularized version of the Type IIA flow. Next, we show that the regularized flow preserves the primitiveness of the data, and reduces to the Type IIA flow if the form $\varphi$ is known to be primitive. Altogether, we obtain the desired short-time existence of the Type IIA flow for primitive data. The uniqueness of the solution will be shown later in \$7.5, as a consequence of the uniqueness of the flows of the corresponding metrics.

\subsection{A coupled flow for $(\varphi, \omega)$}

More precisely, we consider a reparametrization of the Type IIA flow by the following time-dependent vector field

$$
V^{k}=e^{u}\left(g^{p q}\left(\Gamma_{p q}^{k}-\left(\Gamma_{0}\right)_{p q}^{k}\right)-g^{l k} u_{l}\right),
$$

where $|\varphi|, u$ and $g$ are defined by

$$
|\varphi|^{2} \frac{\omega^{3}}{3 !}=\varphi \wedge \hat{\varphi}, \quad u=\log |\varphi|^{2}, \quad g_{i j}=-|\varphi|^{-2} \varphi_{i a b} \varphi_{j c d} \omega^{a c} \omega^{b d}
$$


and $\Gamma$ and $\Gamma_{0}$ are Christoffel symbols associated to the evolving metric $g$ and the initial metric $g_{0}$. Under a reparametrization by the diffeomorphisms generated by the vector field $V^{k}$, the given symplectic form in the Type IIA flow would become time-dependent and evolve by its Lie derivative. It is convenient to change notation slightly, and denote the given symplectic form by $\omega_{0}$ while reserving $\omega=\omega(t)$ for the evolving symplectic form. This consideration inspires us to consider the following coupled flow for the pair $(\varphi, \omega)$,

$$
\left\{\begin{array}{l}
\partial_{t} \varphi=d \Lambda d\left(|\varphi|^{2} \hat{\varphi}\right)+d\left(\iota_{V} \varphi\right) \\
\partial_{t} \omega=d\left(\iota_{V} \omega\right)
\end{array}\right.
$$

with initial data $\varphi(0)=\varphi_{0}, \omega(0)=\omega_{0}$, where $\varphi_{0}$ would be a closed primitive positive 3 -form with respect to $\omega_{0}$. Although the initial metric $g_{0}$ is almost Kähler, a priori we should not assume that $\varphi(t)$ is primitive with respect to $\omega(t)$, hence $g(t)$ a priori may not even be almost Hermitian.

Our first task is to work out the eigenvalues of the principal symbol for this coupled flow. Note that because of the coupling, the principal symbol of (5.2) is now a linear operator acting on both $\delta \varphi$ and $\delta \omega$, and not just on $\delta \varphi$, and we may no longer assume that $\delta \varphi$ is primitive. It is easy to see that the principal symbol of the linearization of (5.2) is determined by

$$
(\delta \varphi, \delta \omega) \rightarrow\left(d \Lambda d \delta\left(|\varphi|^{2} \hat{\varphi}\right)+d\left(\iota_{\delta V} \varphi\right), d\left(\iota_{\delta V} \omega\right)\right)
$$

Now the leading order term in $\delta V$ is

$$
e^{u} g^{p q} g^{k l}\left(\nabla_{p}(\delta g)_{l q}-\frac{1}{2} \nabla_{l}(\delta g)_{p q}\right)-g^{l k} \nabla_{l} \delta\left(e^{u}\right)
$$

so if we define the vector field $W_{\xi}$ by

$$
W_{\xi}^{k}=|\varphi|^{2} g^{p q} g^{k l}\left(\xi_{p}(\delta g)_{l q}-\frac{1}{2} \xi_{l}(\delta g)_{p q}\right)-g^{l k} \xi_{l} \delta|\varphi|^{2}
$$

it follows immediately that the principal symbol of the linearized operator is

$$
(\delta \varphi, \delta \omega) \mapsto\left(\xi \wedge \Lambda\left(\xi \wedge \delta\left(|\varphi|^{2} \hat{\varphi}\right)\right)+\xi \wedge \iota_{W_{\xi}} \varphi, \xi \wedge \iota_{W_{\xi}} \omega\right)
$$

with integrability conditions $\xi \wedge \delta \varphi=\xi \wedge \delta \omega=0$.

We work out more explicitly the symbol at a point $(\varphi, \omega)$ where $\varphi$ is primitive with respect to $\omega$. In this case, we may choose an orthonormal basis of $g$ such that

$$
\begin{aligned}
& \omega=e^{12}+e^{34}+e^{56} \\
& \varphi=\frac{|\varphi|}{2}\left(e^{135}-e^{146}-e^{245}-e^{236}\right), \quad \hat{\varphi}=\frac{|\varphi|}{2}\left(e^{136}+e^{145}+e^{235}-e^{246}\right) .
\end{aligned}
$$


Without loss of generality, we may further assume that $\xi=e^{1}$ and $|\varphi|=1$. In this case, we can write $\delta \varphi=e^{1} \wedge \gamma$ and $\delta \omega=e^{1} \wedge \alpha$ for some 2-form $\gamma$ and 1-form $\alpha$ such that $\alpha, \gamma \in \Lambda^{*}\left\{e^{2}, \ldots, e^{6}\right\}$. It is straightforward to check that

$$
\begin{aligned}
\delta|\varphi|^{2} & =2(\delta \varphi, \varphi)-|\varphi|^{2}(\delta \omega, \omega)=\left(\gamma, e^{35}-e^{46}\right)-\left(\alpha, e^{2}\right), \\
\delta\left(|\varphi|^{2} \hat{\varphi}\right) & =-|\varphi|^{2} J(\delta \varphi)-2(\delta \varphi, \hat{\varphi}) \varphi+4(\delta \varphi, \varphi) \hat{\varphi}-|\varphi|^{2}(\delta \omega, \omega) \hat{\varphi} \\
& =e^{2} \wedge J \gamma-\left(\gamma, e^{36}+e^{45}\right) \varphi+\left(2\left(\gamma, e^{35}-e^{46}\right)-\left(\alpha, e^{2}\right)\right) \hat{\varphi} \\
W_{\xi}^{k} & =(\delta g)_{k 1}-\delta_{1}^{k}\left(\frac{1}{2} \operatorname{tr}_{g} \delta g+\left(\gamma, e^{35}-e^{46}\right)-\left(\alpha, e^{2}\right)\right) .
\end{aligned}
$$

We see that the key is to compute $\delta g$, especially $(\delta g)_{k 1}$. By definition of $\tilde{g}$ and straightforward calculation, we have

$$
\begin{aligned}
& (\delta \tilde{g})_{11}=2\left(\gamma, e^{35}-e^{46}\right), \quad(\delta \tilde{g})_{22}=0, \quad(\delta \tilde{g})_{12}=-\left(\gamma, e^{36}+e^{45}\right), \\
& (\delta \tilde{g})_{33}=(\delta \tilde{g})_{55}=2\left(\gamma, e^{35}\right)-\left(\alpha, e^{2}\right), \quad(\delta \tilde{g})_{44}=(\delta \tilde{g})_{66}=-2\left(\gamma, e^{46}\right)-\left(\alpha, e^{2}\right), \\
& (\delta \tilde{g})_{13}=\left(\gamma, e^{26}\right)+\frac{1}{2}\left(\alpha, e^{4}\right), \quad(\delta \tilde{g})_{14}=\left(\gamma, e^{25}\right)-\frac{1}{2}\left(\alpha, e^{3}\right), \\
& (\delta \tilde{g})_{15}=-\left(\gamma, e^{24}\right)+\frac{1}{2}\left(\alpha, e^{6}\right), \quad(\delta \tilde{g})_{16}=-\left(\gamma, e^{23}\right)-\frac{1}{2}\left(\alpha, e^{5}\right), \\
& (\delta g)_{i j}=(\delta \tilde{g})_{i j}-\left(\left(\gamma, e^{35}-e^{46}\right)-\left(\alpha, e^{2}\right)\right) \delta_{i j} .
\end{aligned}
$$

It follows that $\operatorname{tr}_{g} \delta g=2\left(\alpha, e^{2}\right)$, hence

$$
\begin{array}{cc}
W_{\xi}^{1}=\left(\alpha, e^{2}\right), & W_{\xi}^{2}=-\left(\gamma, e^{36}+e^{45}\right) \\
W_{\xi}^{3}=\left(\gamma, e^{26}\right)+\frac{1}{2}\left(\alpha, e^{4}\right), & W_{\xi}^{4}=\left(\gamma, e^{25}\right)-\frac{1}{2}\left(\alpha, e^{3}\right), \\
W_{\xi}^{5}=-\left(\gamma, e^{24}\right)+\frac{1}{2}\left(\alpha, e^{6}\right), & W_{\xi}^{6}=-\left(\gamma, e^{23}\right)-\frac{1}{2}\left(\alpha, e^{5}\right) .
\end{array}
$$

Consequently we find that

$$
\begin{aligned}
\xi \wedge \iota_{W_{\xi}} \varphi= & \frac{1}{2} e^{1} \wedge\left[\left(\gamma_{36}+\gamma_{45}\right)\left(e^{36}+e^{45}\right)+\left(\gamma_{26}+\frac{\alpha_{4}}{2}\right) e^{26}+\left(\gamma_{25}-\frac{\alpha_{3}}{2}\right) e^{25}\right. \\
& \left.+\left(\gamma_{24}-\frac{\alpha_{6}}{2}\right) e^{24}+\left(\gamma_{23}+\frac{\alpha_{5}}{2}\right) e^{23}+\alpha_{2}\left(e^{35}-e^{46}\right)\right] \\
\xi \wedge \iota_{W_{\xi}} \omega= & \frac{1}{2} e^{1} \wedge\left(\alpha+2 \alpha_{2} e^{2}-2\left(\gamma_{25} e^{3}-\gamma_{26} e^{4}-\gamma_{23} e^{5}+\gamma_{24} e^{6}\right)\right) .
\end{aligned}
$$


If we further write $\gamma=e^{2} \wedge \beta+\lambda, \alpha=\alpha_{2} e^{2}+\mu$, where $\beta, \mu, \lambda \in \Lambda^{*}\left\{e^{3}, \ldots, e^{6}\right\}$, we have

$$
\begin{aligned}
\xi \wedge \iota_{W_{\xi}} \varphi= & \frac{1}{2} e^{1} \wedge\left[\left(\lambda_{36}+\lambda_{45}\right)\left(e^{36}+e^{45}\right)+e^{2} \wedge \beta-\frac{1}{2} e^{2} \wedge \iota_{\mu}\left(e^{35}-e^{46}\right)\right. \\
& \left.+\alpha_{2}\left(e^{35}-e^{46}\right)\right], \\
\xi \wedge \iota_{W_{\xi}} \omega= & \frac{1}{2} e^{1} \wedge\left(\mu+2 \alpha_{2} e^{2}+2 \iota_{\beta}\left(e^{35}-e^{46}\right)\right), \\
\xi \wedge \Lambda\left(\xi \wedge \delta\left(|\varphi|^{2} \hat{\varphi}\right)=\right. & e^{1} \wedge\left[J \lambda+\frac{\lambda_{36}+\lambda_{45}}{2}\left(e^{36}+e^{45}\right)+\left(\lambda_{35}-\lambda_{46}-\frac{\alpha_{2}}{2}\right)\left(e^{35}-e^{46}\right)\right],
\end{aligned}
$$

It follows that the principal symbol is the linear map

$$
\left(\beta, \lambda, \alpha_{2}, \mu\right) \mapsto\left(\frac{\beta}{2}-\frac{\iota_{\mu}}{4}\left(e^{35}-e^{46}\right), \lambda, \alpha_{2}, \frac{\mu}{2}+\iota_{\beta}\left(e^{35}-e^{46}\right)\right)
$$

This matrix is only positive semi-definite. The part $\left(\lambda, \alpha_{2}\right) \mapsto\left(\lambda, \alpha_{2}\right)$ is the identity map. However the other part

$$
(\beta, \mu) \mapsto\left(\frac{\beta}{2}-\frac{\iota_{\mu}}{4}\left(e^{35}-e^{46}\right), \frac{\mu}{2}+\iota_{\beta}\left(e^{35}-e^{46}\right)\right)
$$

has eigenvalues 0 and 1 , both of multiplicities 4. So the coupled flow (5.2) for $(\varphi, \omega)$ is still not strictly parabolic.

\subsection{A parabolic regularization of the coupled flow}

To solve this problem, we add an extra term on the right hand side of the evolution equation of $\varphi$ in (5.2). This term takes the form

$$
-B d J d\left(|\varphi|^{2} \Lambda \hat{\varphi}\right)
$$

where $B$ is a constant to be determined. In fact, $\Lambda(\hat{\varphi})$ is expected to be zero along the flow as $\varphi$ should always be primitive. Again let us consider the linearization of $|\varphi|^{2} \Lambda \hat{\varphi}$ at a primitive pair $(\omega, \varphi)$ and we may assume that $|\varphi|^{2}=1$ at the point of linearization. It follows that

$$
\delta\left(|\varphi|^{2} \Lambda \hat{\varphi}\right)=(\delta \Lambda)(\hat{\varphi})-\Lambda(J \delta \varphi)
$$

As before, we may assume that $\xi=e^{1}$ and $\delta \varphi=e^{1} \wedge\left(e^{2} \wedge \beta+\lambda\right)$ and $\delta \omega=e^{1} \wedge\left(\alpha_{2} e^{2}+\mu\right)$. The principal symbol for the extra term is

$$
B e^{12} \wedge J[(\delta \Lambda)(\hat{\varphi})-\Lambda(J \delta \varphi)]
$$


The second term is easy to compute:

$$
-B e^{12} \wedge J(\Lambda(J \delta \varphi))=B e^{12} \wedge \beta
$$

The first term is more complicated, notice that

$$
B(\delta \Lambda)(\hat{\varphi})_{k}=-\frac{B}{2} \omega^{j s}(\delta \omega)_{s t} \omega^{t i} \hat{\varphi}_{i j k}=B \sum_{t=3}^{6} \mu_{t} \omega^{t i} \hat{\varphi}_{2 i k}
$$

therefore by straightforward calculation, this part of the principal symbol is equivalent to the linear map

$$
\mu \mapsto \frac{B}{2} \iota_{\mu}\left(e^{35}-e^{46}\right) .
$$

Therefore the principal symbol for the full evolution equation is equivalent to the linear map

$$
\left(\beta, \lambda, \alpha_{2}, \mu\right) \mapsto\left(\frac{\beta}{2}(1+2 B)+\frac{2 B-1}{4} \iota_{\mu}\left(e^{35}-e^{46}\right), \lambda, \alpha_{2}, \frac{\mu}{2}+\iota_{\beta}\left(e^{35}-e^{46}\right)\right) .
$$

If $B>0$, then all the eigenvalues of the above matrix are positive. In this sense, the coupled flow with the additional $B$ term is parabolic.

Lemma 11 Consider the flow

$$
\begin{aligned}
& \partial_{t} \varphi=d \Lambda d\left(|\varphi|^{2} \hat{\varphi}\right)-B d J d\left(|\varphi|^{2} \Lambda \hat{\varphi}\right)+d\left(\iota_{V} \varphi\right), \\
& \partial_{t} \omega=d\left(\iota_{V} \omega\right),
\end{aligned}
$$

for any fixed, strictly positive constant B. Then for any initial value $\varphi_{0}$ which is a closed, positive, and primitive form with respect to the initial symplectic form $\omega_{0}$, the flow exists and is smooth at least on some interval $[0, T)$ with $T>0$. Clearly the flow preserves both the closedness of both the form $\varphi$ and the symplectic form $\omega$.

Proof. Let $d$ be the exterior derivative. The preceding fact that the eigenvalues of the principal symbol of the flow (5.10) when restricted to closed and primitive forms are positive means that the flow (5.10) together with $d$ as the integrability operator satisfies the condition of the Hamilton-Nash-Moser theorem ([29], Theorem 5.1). This theorem implies the short-time existence and uniqueness of the flow (5.10). Q.E.D.

It should be noted that we need to treat $\varphi$ and $\omega$ as tensors evolving independently at this moment, therefore we cannot assume that $\varphi$ is primitive with respect to $\omega$ (though we shall prove it is indeed the case later). Consequently the metric $g$ defined above is not necessarily compatible with $J$ or $\omega$ : we only know it is a Riemannian metric. As (5.10) preserves the closedness of $\varphi$ and $\omega$, by performing the reverse reparametrization, we obtain immediately 
Lemma 12 Fix any fixed positive constant B. Then the flow of 3-forms $\varphi$

$$
\partial_{t} \varphi=d \Lambda d\left(|\varphi|^{2} \hat{\varphi}\right)-B d J d\left(|\varphi|^{2} \Lambda \hat{\varphi}\right)
$$

admits a closed smooth solution $\varphi$ on some interval $[0, T)$ with $T>0$, for any initial value $\varphi_{0}$ which is a smooth closed, positive, and primitive form with respect to the symplectic form $\omega_{0}$.

\subsection{Preservation of the primitiveness condition}

Next we shall show that, if the initial data $\varphi_{0}$ is primitive in the flow (5.11), then $\varphi(t)$ remains primitive for all time. Since $\varphi$ primitive implies that $\hat{\varphi}$ is also primitive, it follows that the terms with coefficient $B$ in (5.11) all drop out, and the flow reduces to the Type IIA flow, establishing Theorem 2 in the case of no sources.

From now on, we take $B=1$. Let $\varphi(t)$ be a solution to (5.11) on $M \times[0, T)$ with $\varphi(0)$ being closed, positive, and primitive. Clearly for any $t, \varphi(t)$ stays closed. Let

$$
\varphi=P+\beta \wedge \omega
$$

be the primitive decomposition of $\varphi$, where $P$ is a primitive 3 -form. It follows that

$$
\beta=\frac{\Lambda \varphi}{2}
$$

We wish to show that $\beta=0$ by the maximum principle. To do so, we need to compute the evolution equation of $\beta$. We fix a background metric $\bar{g}=g(0)$ which is compatible

with $\omega$. We denote by $\bar{\nabla}$ the covariant derivatives with respect to $\bar{g}$. Since $\varphi$ is closed, $d \beta$ is primitive, and thus

$$
\omega^{j k} \bar{\nabla}_{j} \beta_{k}=0 .
$$

Furthermore,

$$
(\Lambda \hat{\varphi})_{k}=\frac{\omega^{j i}}{2} \hat{\varphi}_{i j k}=-\frac{\omega^{j i}}{2} \varphi_{i, j, J k}=-(\Lambda \varphi)_{J k},
$$

hence there exists a primitive 3 -form $\hat{P}$ such that the primitive decomposition for $\hat{\varphi}$ is

$$
\hat{\varphi}=\hat{P}-J \beta \wedge \omega
$$

Using this decomposition for $\hat{\varphi}$, we can derive the evolution equation for $\beta$,

$$
\partial_{t} \beta=-d \Lambda d\left(|\varphi|^{2} J \beta\right)+\Lambda\left(d J d\left(|\varphi|^{2} J \beta\right)\right) .
$$


We know that $\varphi, J$, and all their covariant derivatives are bounded in $M \times[0, \tau]$ for any $\tau<T$, therefore we can write (5.15) in the form

$$
\partial_{t} \beta=|\varphi|^{2}\left(-d \Lambda d(J \beta)+\Lambda(d J d J \beta)+\bar{\nabla} \beta * S_{1}+\beta * S_{2}\right),
$$

where $S_{1}$ and $S_{2}$ are bounded tensors on $M \times[0, \tau]$ and $*$ represents certain contraction of indices. We need to compute the leading term of $\beta$ in (5.16). Notice that $(J \beta)_{j}=J^{p}{ }_{j} \beta_{p}$, SO

$$
\begin{aligned}
d(J \beta)_{j k}= & J^{p} \bar{\nabla}_{j} \beta_{p}-J^{p}{ }_{j} \bar{\nabla}_{k} \beta_{p}+O(\beta), \\
\Lambda d(J \beta)= & \omega^{k j} J^{p}{ }_{k} \bar{\nabla}_{j} \beta_{p}+O(\beta), \\
(d \Lambda d(J \beta))_{l}= & \omega^{J p, j} \bar{\nabla}_{l} \bar{\nabla}_{j} \beta_{p}+O(\beta, \bar{\nabla} \beta), \\
(J d J \beta)_{j k}= & J^{s}{ }_{j} J_{k}^{t}\left(J^{p}{ }_{t} \bar{\nabla}_{s} \beta_{p}-J^{p}{ }_{s} \bar{\nabla}_{t} \beta_{p}+O(\beta)\right) \\
= & J^{t}{ }_{k} \bar{\nabla}_{t} \beta_{j}-J^{t}{ }_{j} \bar{\nabla}_{t} \beta_{k}+O(\beta), \\
(d J d J \beta)_{j k l}= & J^{t}{ }_{k}\left(\bar{\nabla}_{l} \bar{\nabla}_{t} \beta_{j}-\bar{\nabla}_{j} \bar{\nabla}_{t} \beta_{l}\right)-J^{t}{ }_{j}\left(\bar{\nabla}_{l} \bar{\nabla}_{t} \beta_{k}-\bar{\nabla}_{k} \bar{\nabla}_{t} \beta_{l}\right) \\
& +J_{l}^{t}{ }_{l}\left(\bar{\nabla}_{j} \bar{\nabla}_{t} \beta_{k}-\bar{\nabla}_{k} \bar{\nabla}_{t} \beta_{j}\right)+O(\beta, \bar{\nabla} \beta), \\
\Lambda(d J d J \beta)_{l}= & \omega^{k j} J_{k}^{t}\left(\bar{\nabla}_{l} \bar{\nabla}_{t} \beta_{j}-\bar{\nabla}_{j} \bar{\nabla}_{t} \beta_{l}\right)+J_{l}^{t} \bar{\nabla}_{t}\left(\omega^{k j} \bar{\nabla}_{j} \beta_{k}\right)+O(\beta, \bar{\nabla} \beta) \\
= & \omega^{J j, p} \bar{\nabla}_{l} \bar{\nabla}_{j} \beta_{p}+\omega^{j, J t} \bar{\nabla}_{j} \bar{\nabla}_{t} \beta_{l}+O(\beta, \bar{\nabla} \beta) .
\end{aligned}
$$

It follows that

$$
\partial_{t} \beta=|\varphi|^{2}(L \beta+O(\beta, \bar{\nabla} \beta))
$$

where $L$ is defined by

$$
(L \beta)_{l}=\left(\omega^{J j, p}+\omega^{j, J p}\right) \bar{\nabla}_{l} \bar{\nabla}_{j} \beta_{p}+\omega^{j, J t} \bar{\nabla}_{j} \bar{\nabla}_{t} \beta_{l} .
$$

We further notice that $\omega^{J j, p}+\omega^{j, J p}=O(\beta), \omega^{j, J t}-\delta^{j t}=O(\beta)$, and as $\beta$ is a smooth function of $\varphi$, we also have $\left|\bar{\nabla}^{2} \beta\right|$ is uniformly bounded. Therefore one can also write

$$
\partial_{t} \beta=|\varphi|^{2}(\bar{\Delta} \beta+O(\beta, \bar{\nabla} \beta)) .
$$

It follows that

$$
\begin{aligned}
\partial_{t}|\beta|_{\bar{g}}^{2} & =2|\varphi|^{2}(\bar{\Delta} \beta, \beta)_{\bar{g}}+O(\beta, \bar{\nabla} \beta) * \beta * S \\
& \leq|\varphi|^{2} \bar{\Delta}\left(|\beta|_{\bar{g}}^{2}\right)-2|\varphi|^{2}|\bar{\nabla} \beta|^{2}+O(\beta, \bar{\nabla} \beta) * \beta * S \\
& \leq|\varphi|^{2} \bar{\Delta}\left(|\beta|_{\bar{g}}^{2}\right)+C|\beta|^{2} .
\end{aligned}
$$

Since $\beta=0$ initially, by maximum principle we know that $\beta=0$ on $[0, \tau]$ for any $\tau<T$. Therefore $\varphi(t)$ is primitive for as long as the flow exists, and $J$ is always compatible with $\omega$. The existence part of Theorem 2 is proved in the case of no sources. 


\section{Type IIA geometry: proof of Theorem 3}

The goal of this section is to work out some properties specific to Type IIA geometry. What is crucial is that the almost complex structure $J_{\varphi}$ in Type IIA strings comes from a closed primitive positive 3-form $\varphi$ via Hitchin's construction. In fact, the closedness of $\varphi$ imposes subtle "higher integrability" conditions on $J_{\varphi}$ which in turn distinguish $J_{\varphi}$ from a generic almost complex structure. This feature gives rise to various identities that are not available in the more general almost-Kähler setting. We begin with the curvature and Nijenhuis tensor on general almost-complex manifolds, and gradually specialize to almost-Kähler manifolds, and then to Type IIA geometry.

\subsection{Curvature tensors on general almost-complex manifolds}

For any affine connection $D$, we define its curvature tensor $R(D)$ and torsion $T(D)$ by

$$
\left[D_{i}, D_{j}\right] X^{m}=R(D)_{i j}{ }^{m} X^{l}-T(D)^{l}{ }_{i j} D_{l} X^{m} .
$$

The curvature tensor with four lower indices is defined in the usual way

$$
R(D)_{i j k l}=R(D)_{i j}{ }^{p} g_{p k} .
$$

As in the case for Levi-Civita connection, we define the Ricci curvature of $D$, also denoted by $R(D)$, by

$$
R(D)_{i k}=g^{j l} R(D)_{i j k l}
$$

Let now $J$ be an almost-complex structure on the Riemannian manifold $M$. In subsequent developments, we shall need both the Levi-Civita connection $\nabla$ and the projected Levi-Civita connection $\mathfrak{D}=\mathfrak{D}^{0}$. Therefore we will reserve the Latin letter $R$ for various curvature tensors associated to $\nabla$ and the German letters $\mathfrak{d}, \mathfrak{T}$, and $\mathfrak{R}$ for Christoffel symbol, torsion, and curvature tensors associated to $\mathfrak{D}$. When we have other Hermitian metrics with decoration like $\tilde{g}$ or $\hat{g}$, we shall decorate the corresponding connections and curvature tensors with the same symbol. Identities for the curvature and torsion of the Chern connection have been worked out in the paper of Tosatti, Weinkove, and Yau [47]. However, they are expressed there in complex frames, and it is difficult for us to apply their formulas, as we shall have to let the almost-complex structure evolve. Thus we develop here a formalism for curvature and torsion identities with the action of $J$ in real coordinate systems.

To pass back and forth from $\nabla$ to $\mathfrak{d}$, we note that from (3.11) and (3.12) that

$$
\begin{aligned}
& \mathfrak{d}^{m}{ }_{i j}=\Gamma^{m}{ }_{i j}-N_{i j}{ }^{m}-V_{i j}{ }^{m}=: \Gamma^{m}{ }_{i j}-A_{i j}{ }^{m}, \\
& \mathfrak{T}^{m}{ }_{i j}=N^{m}{ }_{i j}-U^{m}{ }_{i j},
\end{aligned}
$$


where $A=V+N$ is of type $(2,0)+(0,2)$, and hence their curvature tensors are related by

$$
R_{i j k l}=\mathfrak{R}_{i j k l}-\left(\mathfrak{D}_{i} A_{j k l}-\mathfrak{D}_{j} A_{i k l}+\mathfrak{T}^{p}{ }_{i j} A_{p k l}+A_{i k}^{p} A_{j l p}-A_{j k}^{p} A_{i l p}\right)
$$

As $R_{i j k l}$ is the curvature tensor of the Levi-Civita connection, it has various symmetries and satisfies the Bianchi identities. On the other hand, since $\mathfrak{D} J=0$, its curvature $\mathfrak{D}$ satisfies

$$
\mathfrak{R}_{i j k l}=\mathfrak{R}_{i, j, J k, J l}
$$

It is easy to deduce from the preceding relation between $R_{i j k l}$ and $\mathfrak{R}_{i j k l}$ how to modify the identity for each curvature if it is replaced by the other.

The projected Levi-Civita connection $\mathfrak{D}$ induces a connection on the canonical bundle of $M$, whose curvature represents the first Chern class (up to a constant) of the almost complex manifold $(M, J)$. To be precise, if we use small Greek letter to denote the index for "holomorphic" tangent bundle $T^{1,0} M$, then

$$
\frac{1}{4 \pi} \mathfrak{R}_{i j k l} \omega^{l k}=\frac{\sqrt{-1}}{2 \pi} \mathfrak{R}_{i j}^{\gamma}{ }_{\gamma} \in\left[c_{1}(M, J)\right],
$$

Since $A$ is of type $(2,0)+(0,2)$, the contraction of its last two indices using $\omega$ or $g$ vanishes, therefore by (6.6) we see that

$$
\mathfrak{R}_{i j k l} \omega^{l k}=R_{i j k l} \omega^{l k}+2 A_{i k}^{p} A_{j l p} \omega^{l k}
$$

is a closed 2-form. We end this subsection by deriving the following formula for Ricci curvature

$$
R_{i j}=-2 g^{k l}\left(\mathfrak{D}_{i} A_{k j l}-\mathfrak{D}_{k} A_{i j l}+\mathfrak{T}^{p}{ }_{i k} A_{p j l}\right)+\frac{1}{2} \omega^{l k} R_{i, J j, k, l}
$$

Indeed, by (6.6) we see that

$$
\begin{aligned}
R_{i, j, J k, J l}-R_{i j k l}= & \left(\mathfrak{D}_{i} A_{j k l}-\mathfrak{D}_{j} A_{i k l}+\mathfrak{T}^{p}{ }_{i j} A_{p k l}+A_{i k}{ }^{p} A_{j l p}-A_{j k}{ }^{p} A_{i l p}\right) \\
& -\left(\mathfrak{D}_{i} A_{j, J k, J l}-\mathfrak{D}_{j} A_{i, J k, J l}+\mathfrak{T}^{p}{ }_{i j} A_{p, J k, J l}+A_{i, J k}{ }^{p} A_{j, J l, p}-A_{j, J k}{ }^{p} A_{i, J l, p}\right) .
\end{aligned}
$$

Recall that $A$ is of type $(2,0)+(0,2)$, so $A_{i j k}=-A_{i, J j, J k}$, therefore we get

$$
R_{i, j, J k, J l}-R_{i j k l}=2\left(\mathfrak{D}_{i} A_{j k l}-\mathfrak{D}_{j} A_{i k l}+\mathfrak{T}^{p}{ }_{i j} A_{p k l}\right)
$$

Let us denote the right hand side of the above equation by $B_{i j k l}$. Then the above equation is equivalent to

$$
\begin{aligned}
-R_{i, j, J k, l}-R_{i, j, k, J l} & =2\left(\mathfrak{D}_{i} A_{j, k, J l}-\mathfrak{D}_{j} A_{i, k, J l}+\mathfrak{T}^{p}{ }_{i j} A_{p, k, J l}\right) \\
& =B_{i, j, J k, l}=B_{i, j, k, J l} .
\end{aligned}
$$


Let $\left\{e_{a}\right\}$ be an orthonormal frame for the given Riemannian metric, and so is the frame $\left\{J e_{a}\right\}$. By definition of Ricci curvature, we have

$$
\begin{aligned}
R_{i, J j} & =\sum_{a} R\left(i, e_{a}, J j, e_{a}\right)=\sum_{a} R\left(i, J e_{a}, J j, J e_{a}\right) \\
& =\sum_{a}\left(R\left(i, J e_{a}, j, e_{a}\right)+B\left(i, J e_{a}, j, e_{a}\right)\right) \\
& =\sum_{a}\left(-R\left(j, e_{a}, J(J i), J e_{a}\right)-B\left(i, J e_{a}, J j, J e_{a}\right)\right) \\
& =\sum_{a}\left(-R\left(j, e_{a}, J i, e_{a}\right)-B\left(j, e_{a}, J i, e_{a}\right)-B\left(i, e_{a}, J j, e_{a}\right)\right) \\
& =-R_{j, J i}-g^{k l}\left(B_{i, k, J j, l}+B_{j, k, J i, l}\right) .
\end{aligned}
$$

On the other hand, by taking trace of (6.12) and using Bianchi identity of $R$, we have

$$
\begin{aligned}
g^{j l} B_{i, j, J k, l} & =-g^{j l}\left(R_{i, j, J k, l}+R_{i, j, k, J l}\right) \\
& =-R_{i, J k}+g^{j l}\left(R_{j, k, i, J l}+R_{k, i, j, J l}\right) \\
& =-R_{i, J k}+g^{j l}\left(R_{k, j, J i, l}+B_{k, j, J i, l}\right)+R_{k i j l} \omega^{l j} \\
& =-R_{i, J k}+R_{k, J i}+g^{j l} B_{k, j, J i, l}+R_{k i j l} \omega^{l j} .
\end{aligned}
$$

(6.13) and (6.14) can be rewritten as

$$
\begin{aligned}
& R_{i, J j}+R_{j, J i}=-g^{k l}\left(B_{i, k, J j, l}+B_{j, k, J i, l}\right), \\
& R_{i, J j}-R_{j, J i}=-g^{k l}\left(B_{i, k, J j, l}-B_{j, k, J i, l}\right)-R_{i j k l} \omega^{l k} .
\end{aligned}
$$

Adding these two equations up we get

$$
R_{i, J j}=-g^{k l} B_{i, k, J j, l}-\frac{1}{2} R_{i j k l} \omega^{l k}
$$

which is equivalent to

$$
\begin{aligned}
R_{i j} & =-g^{k l} B_{i k j l}+\frac{1}{2} \omega^{l k} R_{i, J j, k, l} \\
& =-2 g^{k l}\left(\mathfrak{D}_{i} A_{k j l}-\mathfrak{D}_{k} A_{i j l}+\mathfrak{T}^{p}{ }_{i k} A_{p j l}\right)+\frac{1}{2} \omega^{l k} R_{i, J j, k, l} .
\end{aligned}
$$

This gives the desired formula.

\subsection{Quadratic expressions in the Nijenhuis tensor}

We shall encounter frequently later quadratic expressions of the Nijenhuis tensor. It is convenient to introduce the following two symmetric tensors quadratic in $N$ :

$$
\begin{aligned}
& \left(N_{+}^{2}\right)_{i j}:=N_{i}^{p k} N_{p k j} \geq 0, \\
& \left(N_{-}^{2}\right)_{i j}:=N^{k p}{ }_{i} N_{p k j} .
\end{aligned}
$$


Since $N$ is skew-symmetric in the last two slots and it satisfies the Bianchi identity (3.8), all the other similar tensors can be expressed as a linear combination of $N_{+}^{2}$ and $N_{-}^{2}$. For example

$$
0 \leq N_{i p k} N_{j}^{p k}=\left(N_{p k i}-N_{k p i}\right)\left(N_{j}^{p k}-N_{j}^{k p}\right)=2\left(N_{+}^{2}\right)_{i j}-2\left(N_{-}^{2}\right)_{i j}
$$

Obviously $g^{i j} N_{i p k} N_{j}^{p k}=|N|^{2}=g^{i j} N^{p k}{ }_{i} N_{p k j}$, so we find that

$$
|N|^{2}=\operatorname{tr} N_{+}^{2}=g^{i j}\left(N_{+}^{2}\right)_{i j}=2 g^{i j}\left(N_{-}^{2}\right)_{i j}=2 \operatorname{tr} N_{-}^{2} .
$$

Also we observe that both $N_{+}^{2}$ and $N_{-}^{2}$ are $J$-invariant in the sense that $\left(N_{ \pm}^{2}\right)_{i j}=\left(N_{ \pm}^{2}\right)_{J i, J j}$. In general, for any symmetric 2-tensor $A=A_{i j}$, we define its $J$-invariant and $J$-antiinvariant parts respectively by

$$
\left(A^{J}\right)_{i j}:=\frac{1}{2}\left(A_{i j}+A_{J i, J j}\right), \quad\left(A^{-J}\right)_{i j}:=\frac{1}{2}\left(A_{i j}-A_{J i, J j}\right) .
$$

In this notation, we have $N_{ \pm}^{2}=\left(N_{ \pm}^{2}\right)^{J}$.

Clearly we have $A=A^{J}+A^{-J}$ and this decomposition is orthogonal with respect to the inner product induced by the metric $g$. Later such a decomposition will play an important role in our calculations.

\subsection{Curvature tensors in almost-Kähler geometry}

In this subsection we restrict ourselves to the case $d \omega=0$, namely the case $(M, J, g)$ is an almost-Kähler manifold. Since $\omega$ is a symplectic form, we know $d^{c} \omega=0$, hence both $U$ and $V$ defined in (3.5) are zero. Therefore (6.4) and (6.5) specialize to $A=N=\mathfrak{T}$. Therefore the previously deduced formula (6.6) becomes

$$
R_{i j k l}=\mathfrak{R}_{i j k l}-\left(\mathfrak{D}_{i} N_{j k l}-\mathfrak{D}_{j} N_{i k l}+N^{p}{ }_{i j} N_{p k l}+N_{i k}^{p} N_{j l p}-N_{j k}{ }^{p} N_{i l p}\right),
$$

thus we have

$$
R_{i j}=\mathfrak{R}_{i j}+\mathfrak{D}^{k} N_{i j k}-\left(N_{-}^{2}\right)_{i j}
$$

Combining (6.10) with (6.16), we also obtain

$$
\begin{aligned}
R_{i j}= & 2 \mathfrak{D}^{k} N_{i j k}-2\left(N_{+}^{2}\right)_{i j}+\frac{1}{2} \omega^{l k} R_{i, J j, k, l} \\
= & 2 \mathfrak{D}^{k} N_{i j k}-2\left(N_{+}^{2}\right)_{i j} \\
& +\frac{1}{2} \omega^{l k}\left(\mathfrak{R}_{i, J j, k, l}-\left(\mathfrak{D}_{i} N_{J j, k, l}-\mathfrak{D}_{J j} N_{i k l}+N^{p}{ }_{i, J j} N_{p k l}+N_{i k}{ }^{p} N_{J j, l, p}-N_{J j, k}{ }^{p} N_{i l p}\right)\right) \\
= & 2 \mathfrak{D}^{k} N_{i j k}-2\left(N_{-}^{2}\right)_{i j}+\frac{1}{2} \omega^{l k} \mathfrak{R}_{i, J j, k, l} .
\end{aligned}
$$


Alternatively

$$
\begin{aligned}
R_{i j}= & 2 \mathfrak{D}^{k} N_{i j k}-2\left(N_{+}^{2}\right)_{i j}+\frac{1}{2} \omega^{l k} R_{k, l, i, J j} \\
= & 2 \mathfrak{D}^{k} N_{i j k}-2\left(N_{+}^{2}\right)_{i j} \\
& +\frac{1}{2} \omega^{l k}\left(\mathfrak{R}_{k, l, i, J j}-\left(\mathfrak{D}_{k} N_{l, i, J j}+N^{p}{ }_{k l} N_{p, i, J j}-\mathfrak{D}_{l} N_{k, i, J j}+N_{k i}{ }^{p} N_{l, J j, p}-N_{l i}{ }^{p} N_{k, J j, p}\right)\right) \\
= & \mathfrak{D}^{k}\left(N_{i j k}+N_{j i k}\right)-\left(N_{+}^{2}\right)_{i j}+\frac{1}{2} \omega^{l k} \mathfrak{R}_{k, l, i, J j} .
\end{aligned}
$$

From (6.53) we can immediately read off that

$$
\begin{aligned}
\left(R^{J}\right)_{i j} & =-\left(N_{+}^{2}\right)_{i j}+\frac{1}{2} \omega^{l k} \mathfrak{R}_{k, l, i, J j}, \\
\left(R^{-J}\right)_{i j} & =\mathfrak{D}^{k}\left(N_{i j k}+N_{j i k}\right) .
\end{aligned}
$$

Taking the trace of (6.18) and plugging in (6.16), we see that

$$
R=\frac{1}{2} \omega^{j i} \omega^{l k} \Re_{i j k l}-|N|^{2}=\frac{1}{2} \omega^{j i} \omega^{l k} R_{i j k l}-2|N|^{2}
$$

In the literature, the expression $\frac{1}{2} \omega^{j i} \omega^{l k} R_{i j k l}$ is sometimes known as the $\star$-scalar curvature. This relation (6.22) was first discovered by Blair-Ianus [4], and Blair [3] together with Oproiu 36.

Combining (6.16) with the symmetry of $R$, we can derive that

$$
\begin{aligned}
\mathfrak{R}_{i j k l}-\mathfrak{R}_{k l i j}= & \mathfrak{D}_{i} N_{j k l}-\mathfrak{D}_{j} N_{i k l}-\mathfrak{D}_{k} N_{l i j}+\mathfrak{D}_{l} N_{k i j} \\
& +N_{i k}{ }^{p} N_{j l p}-N_{j k}{ }^{p} N_{i l p}-N_{k i}{ }^{p} N_{l j p}+N_{l i}{ }^{p} N_{k j p} .
\end{aligned}
$$

As $\mathfrak{R}_{i j k l}=\mathfrak{R}_{i, j, J k, J l}$, by making use of $(\underline{6.23})$, we get

$$
\begin{aligned}
& \mathfrak{R}_{J i, J j, k, l}-\mathfrak{R}_{i j k l}=\mathfrak{R}_{J i, J j, k, l}-\mathfrak{R}_{k, l, J i, J j}+\mathfrak{R}_{k l i j}-\mathfrak{R}_{i j k l} \\
= & \mathfrak{D}_{J i} N_{J j, k, l}-\mathfrak{D}_{J j} N_{J i, k, l}-\mathfrak{D}_{i} N_{j k l}+\mathfrak{D}_{j} N_{i k l}+2 \mathfrak{D}_{k} N_{l i j}-2 \mathfrak{D}_{l} N_{k i j} .
\end{aligned}
$$

Notice that the LHS of (6.24) does not change if one replace $k$ and $l$ by $J k$ and $J l$ respectively, so we get an interesting identity satisfied by $\mathfrak{D} N$

$$
\begin{aligned}
& \mathfrak{D}_{J i} N_{J j, k, l}-\mathfrak{D}_{J j} N_{J i, k, l}-\mathfrak{D}_{J k} N_{J l, i, j}+\mathfrak{D}_{J l} N_{J k, i, j} \\
= & \mathfrak{D}_{i} N_{j k l}-\mathfrak{D}_{j} N_{i k l}-\mathfrak{D}_{k} N_{l i j}+\mathfrak{D}_{l} N_{k i j},
\end{aligned}
$$

which allows us to rewrite one partial derivative of $N$ in terms of some other combination of partial derivatives.

In the same vein we can derive the Bianchi-type identity for $\mathfrak{R}$

$$
\begin{aligned}
& \mathfrak{R}_{i j k l}+\mathfrak{R}_{j k i l}+\mathfrak{R}_{k i j l} \\
= & -\mathfrak{D}_{i} N_{l j k}-\mathfrak{D}_{j} N_{l k i}-\mathfrak{D}_{k} N_{l i j}+N^{p}{ }_{i j} N_{l k p}+N^{p}{ }_{j k} N_{l i p}+N^{p}{ }_{k i} N_{l j p} .
\end{aligned}
$$


Equation (6.24) accounts for the $(2,0)+(0,2)$-part of the curvature tensor $\mathfrak{R}$. If we use Greek letters for barred and unbarred directions, then (6.24) can be translated into

$$
\begin{aligned}
\mathfrak{R}_{\alpha \beta \bar{\gamma} \delta} & =\mathfrak{D}_{\alpha} N_{\beta \bar{\gamma} \delta}-\mathfrak{D}_{\beta} N_{\alpha \bar{\gamma} \delta}-\mathfrak{D}_{\bar{\gamma}} N_{\delta \alpha \beta}+\mathfrak{D}_{\delta} N_{\bar{\gamma} \alpha \beta} \\
& =-\mathfrak{D}_{\bar{\gamma}} N_{\delta \alpha \beta}, \\
\mathfrak{R}_{* * \gamma \delta} & =\mathfrak{R}_{* * \bar{\gamma} \bar{\delta}}=0,
\end{aligned}
$$

which is the content of (2.17) in [47]. Replace $i, j, k, l$ by $\bar{\alpha}, \beta, \bar{\gamma}, \delta$ respectively in the Bianchi-type identity (6.26), we get

$$
\mathfrak{R}_{\bar{\alpha} \beta \bar{\gamma} \delta}-\mathfrak{R}_{\bar{\gamma} \beta \bar{\alpha} \delta}=\mathfrak{R}_{\bar{\alpha} \beta \bar{\gamma} \delta}+\mathfrak{R}_{\beta \bar{\gamma} \bar{\alpha} \delta}+\mathfrak{R}_{\bar{\gamma} \bar{\alpha} \beta \delta}=N_{\bar{\gamma} \bar{\alpha}}^{p} N_{\delta \beta p}=N_{\bar{\gamma} \bar{\alpha}}^{\lambda} N_{\delta \beta \lambda} .
$$

This is the content of (2.16) in [47].

\subsection{The holonomy of Type IIA geometry}

We now restrict ourselves further to Type IIA geometry, namely a triple $(M, \omega, \varphi)$ where $(M, \omega)$ is a symplectic 6 -manifold and $\varphi$ is a closed positive $\omega$-primitive 3 -form.

Our first task is to prove Theorem 3(a). Recall that $|\varphi|$ is the norm of $\varphi$ with respect to the metric $g_{\varphi}$, and that we have defined the metric $\tilde{g}_{\varphi}$ by $\tilde{g}_{\varphi}=|\varphi|^{2} g_{\varphi}$. It is not hard to see that

$$
|\varphi|_{\tilde{g}}=|\varphi|^{-2}
$$

Henceforth we shall denote $g_{\varphi}, \tilde{g}_{\varphi}$, and $J_{\varphi}$ by just $g, \tilde{g}$, and $J$ for simplicity. It is clear that $J$ is compatible with $\tilde{g}$ and the corresponding Kähler form $\tilde{\omega}=|\varphi|^{2} \omega$ satisfies

$$
d \tilde{\omega}=-\alpha \wedge \tilde{\omega}, \quad d^{c} \tilde{\omega}=J \alpha \wedge \tilde{\omega}
$$

where

$$
\alpha=d \log |\varphi|_{\tilde{g}}=-d \log |\varphi|^{2}
$$

It follows from (3.15) that

$$
\begin{aligned}
U_{i j k} & =\frac{1}{4}\left(2 \alpha_{J i} \tilde{\omega}_{j k}+\alpha_{J j} \tilde{\omega}_{k i}+\alpha_{J k} \tilde{\omega}_{i j}+\alpha_{j} \tilde{g}_{k i}-\alpha_{k} \tilde{g}_{i j}\right), \\
V_{i j k} & =\frac{1}{4}\left(\alpha_{J j} \tilde{\omega}_{k i}+\alpha_{J k} \tilde{\omega}_{i j}-\alpha_{j} \tilde{g}_{k i}+\alpha_{k} \tilde{g}_{i j}\right) .
\end{aligned}
$$

We need now the following lemmas for computational purposes.

\section{Lemma 13}

Let $\mu$ be any differential form, $D$ any affine connection, $T=T(D)$ the torsion tensor associated to $D$. Then we have the following formula

$$
d \mu=d x^{j} \wedge D_{j} \mu+T \bigotimes \mu,
$$


where $\otimes$ is a multiplication operation linear in both factors. We only need the explicit expression of $\otimes$ when $\mu$ is a 3-form, in which case $T \otimes \mu$ is a 4-form given by

$$
(T \otimes \mu)_{i j k l}=T^{p}{ }_{i j} \mu_{p k l}+T^{p}{ }_{k l} \mu_{p i j}-T^{p}{ }_{i k} \mu_{p j l}-T^{p}{ }_{j l} \mu_{p i k}+T^{p}{ }_{i l} \mu_{p j k}+T^{p}{ }_{j k} \mu_{p i l},
$$

as well as the case $\mu$ is a 2-form, where $T \nabla \mu$ is a 3-form of the form

$$
(T \otimes \mu)_{i j k}=T^{p}{ }_{i j} \mu_{p k}+T^{p}{ }_{j k} \mu_{p i}+T_{k i}^{p} \mu_{p j} .
$$

Proof: We give the proof of (6.32) and leave (6.31) to the reader. For $\mu=\frac{1}{2} \mu_{i j} d x^{i} \wedge d x^{j}$, we have

$$
d \mu=\frac{1}{2} \partial_{\alpha} \mu_{i j} d x^{\alpha} \wedge d x^{i} \wedge d x^{j} .
$$

We write $D_{i} W_{j}=\partial_{i} W_{j}-\Gamma(D)^{k}{ }_{i j} W_{k}$, and obtain

$$
d \mu=\frac{1}{2}\left(D_{k} \mu_{i j}+\Gamma(D)^{\beta}{ }_{k i} \mu_{\beta j}+\Gamma(D)^{\beta}{ }_{k j} \mu_{i \beta}\right) d x^{k} \wedge d x^{i} \wedge d x^{j} .
$$

This becomes

$$
\begin{aligned}
d \mu= & d x^{k} \wedge D_{k} \mu+\frac{1}{3 !}\left(\Gamma(D)^{\beta}{ }_{k i} \mu_{\beta j}+\Gamma(D)^{\beta}{ }_{j k} \mu_{\beta i}+\Gamma(D)^{\beta}{ }_{i j} \mu_{\beta k}\right) d x^{k} \wedge d x^{i} \wedge d x^{j} \\
& +\frac{1}{3 !}\left(\Gamma(D)^{\beta}{ }_{k j} \mu_{i \beta}+\Gamma(D)^{\beta}{ }_{i k} \mu_{j \beta}+\Gamma(D)^{\beta}{ }_{j i} \mu_{k \beta}\right) d x^{k} \wedge d x^{i} \wedge d x^{j}
\end{aligned}
$$

which leads to

$$
d \mu=d x^{k} \wedge D_{k} \mu+\frac{1}{3 !}\left(T_{k i}^{\beta} \mu_{\beta j}+T^{\beta}{ }_{j k} \mu_{\beta i}+T^{\beta}{ }_{i j} \mu_{\beta k}\right) d x^{k} \wedge d x^{i} \wedge d x^{j} .
$$

Q.E.D.

\section{Lemma 14}

In the notation in Lemma 13, the 4-form $N \otimes \varphi$ is of type $(2,2)$.

Proof of the Lemma: By Lemma 13, we know that

$$
(N \otimes \varphi)_{i j k l}=N^{p}{ }_{i j} \varphi_{p k l}+N_{k l}^{p} \varphi_{p i j}-N^{p}{ }_{i k} \varphi_{p j l}-N^{p}{ }_{j l} \varphi_{p i k}+N^{p}{ }_{i l} \varphi_{p j k}+N^{p}{ }_{j k} \varphi_{p i l} .
$$

Since $N \in A^{0,2}(T M)$ and $\varphi$ satisfies Lemma [6, we find

$$
\begin{aligned}
(J(N \otimes \varphi))_{i j k l}= & N^{p}{ }_{J i, J j} \varphi_{p, J k, J l}+N^{p}{ }_{J k, J l} \varphi_{p, J i, J j}-N^{p}{ }_{J i, J k} \varphi_{p, J j, J l} \\
& -N^{p}{ }_{J j, J l} \varphi_{p, J i, J k}+N^{p}{ }_{J i, J l} \varphi_{p, J j, J k}+N^{p}{ }_{J j, J k} \varphi_{p, J i, J l} \\
= & N^{p}{ }_{i j} \varphi_{p k l}+N^{p}{ }_{k l} \varphi_{p i j}-N^{p}{ }_{i k} \varphi_{p j l}-N^{p}{ }_{j l} \varphi_{p i k}+N^{p}{ }_{i l} \varphi_{p j k}+N^{p}{ }_{j k} \varphi_{p i l} \\
= & (N \otimes \varphi)_{i j k l} .
\end{aligned}
$$

As $J$ acts on $(3,1)+(1,3)$-forms as -1 and acts on $(2,2)$-forms as 1 , we deduce that $N \otimes \varphi$ is a $(2,2)$-form. Q.E.D. 


\section{Lemma 15}

Using the notation in Lemma 13, we have

$$
\begin{aligned}
& d^{c} \tilde{\omega} \nabla \varphi=\mathcal{M}\left(d^{c} \tilde{\omega}\right) \otimes \varphi=2 \alpha \wedge \varphi, \\
& d^{c} \tilde{\omega} \nabla \hat{\varphi}=\mathcal{M}\left(d^{c} \tilde{\omega}\right) \nabla \hat{\varphi}=2 \alpha \wedge \hat{\varphi} .
\end{aligned}
$$

Proof. As we have seen $d^{c} \tilde{\omega}=J \alpha \wedge \tilde{\omega}$, so the first term in $\left(d^{c} \tilde{\omega}\right) \otimes \varphi$ is

$$
\begin{aligned}
\left(d^{c} \tilde{\omega}\right)^{p}{ }_{i j} \varphi_{p k l} & =\tilde{g}^{p q}\left((J \alpha)_{q} \tilde{\omega}_{i j}+(J \alpha)_{i} \tilde{\omega}_{j q}+(J \alpha)_{j} \tilde{\omega}_{q i}\right) \varphi_{p k l} \\
& =\tilde{g}^{p q} \alpha_{J q} \tilde{\omega}_{i j} \varphi_{p k l}+\alpha_{J i} \varphi_{J j, k, l}-\alpha_{J j} \varphi_{J i, k, l} \\
& =\tilde{g}^{p q} \alpha_{J q} \tilde{\omega}_{i j} \varphi_{p k l}-\alpha_{J i} \hat{\varphi}_{j k l}+\alpha_{J j} \hat{\varphi}_{i k l} .
\end{aligned}
$$

Hence

$$
\begin{aligned}
\left(d^{c} \tilde{\omega} \nabla \varphi\right)_{i j k l}= & \tilde{g}^{p q} \alpha_{J q}\left(\tilde{\omega}_{i j} \varphi_{p k l}+\tilde{\omega}_{k l} \varphi_{p i j}-\tilde{\omega}_{i k} \varphi_{p j l}-\tilde{\omega}_{j l} \varphi_{p i k}+\tilde{\omega}_{i l} \varphi_{p j k}+\tilde{\omega}_{j k} \varphi_{p i l}\right) \\
& -\alpha_{J i} \hat{\varphi}_{j k l}+\alpha_{J j} \hat{\varphi}_{i k l}-\alpha_{J k} \hat{\varphi}_{l i j}+\alpha_{J l} \hat{\varphi}_{k i j}+\alpha_{J i} \hat{\varphi}_{k j l}-\alpha_{J k} \hat{\varphi}_{i j l} \\
& +\alpha_{J j} \hat{\varphi}_{l i k}-\alpha_{J l} \hat{\varphi}_{j i k}-\alpha_{J i} \hat{\varphi}_{l j k}+\alpha_{J l} \hat{\varphi}_{i j k}-\alpha_{J j} \hat{\varphi}_{i l k}+\alpha_{J i} \hat{\varphi}_{j l k} \\
= & \tilde{g}^{p q} \alpha_{J q}\left(\tilde{\omega}_{i p} \varphi_{j k l}-\tilde{\omega}_{j p} \varphi_{i k l}+\tilde{\omega}_{k p} \varphi_{i j l}-\tilde{\omega}_{l p} \varphi_{i j k}\right)-3(J \alpha \wedge \hat{\varphi})_{i j k l} \\
= & \alpha_{J q}\left(J^{q}{ }_{i j k l}-J^{q}{ }_{j} \varphi_{i k l}+J^{q}{ }_{k} \varphi_{i j l}-J^{q} \varphi_{i j k}\right)+3(\alpha \wedge \varphi)_{i j k l} \\
= & 2(\alpha \wedge \varphi)_{i j k l} .
\end{aligned}
$$

In this proof we only used the fact that $\varphi$ is primitive (4.14) so in the same manner we have

$$
d^{c} \tilde{\omega} \otimes \hat{\varphi}=2 \alpha \wedge \hat{\varphi}
$$

The other identities can be proved similarly. Q.E.D.

Now we are ready to prove Theorem 3 (a).

Since $\tilde{\mathfrak{D}} J=0$, there exists a complex-valued 1 -form $\theta=\alpha+\sqrt{-1} \beta$ such that

$$
\tilde{\mathfrak{D}} \Omega=\theta \otimes \Omega .
$$

Taking its real and imaginary parts, we get

$$
\begin{aligned}
& \tilde{\mathfrak{D}} \varphi=\alpha \otimes \varphi-\beta \otimes \hat{\varphi} \\
& \tilde{\mathfrak{D}} \hat{\varphi}=\beta \otimes \varphi+\alpha \otimes \hat{\varphi} .
\end{aligned}
$$

The 1 -form $\alpha$ is very easy to find: as $\tilde{\mathfrak{D}} \tilde{g}=0$, we know that

$$
d|\varphi|_{\tilde{g}}^{2}=\tilde{\mathfrak{D}} \tilde{g}(\varphi, \varphi)=2 \tilde{g}(\tilde{\mathfrak{D}} \varphi, \varphi)=2|\varphi|_{\tilde{g}}^{2} \alpha
$$

hence we conclude that

$$
\alpha=\frac{1}{2} d \log |\varphi|_{\tilde{g}}^{2}=d \log |\varphi|_{\tilde{g}}=-d \log |\varphi|^{2},
$$


which is the exactly same expression we assigned to $\alpha$ in (6.27). To find $\beta$, we plug (6.39) in (6.30) to get

$$
0=d \varphi=\alpha \wedge \varphi-\beta \wedge \hat{\varphi}+\tilde{\mathfrak{T}} \otimes \varphi
$$

Apply (3.12) to the Hermitian metric $\tilde{g}$ with $t=0$, we get

$$
\tilde{\mathfrak{T}}=N-U
$$

where $U=\frac{1}{4}\left(d^{c} \tilde{\omega}+\mathcal{M}\left(d^{c} \tilde{\omega}\right)\right)$. According to Lemma 15 we have

$$
\begin{aligned}
\tilde{\mathfrak{T}} \otimes \varphi & =N \otimes \varphi-\frac{1}{4} d^{c} \tilde{\omega} \nabla \varphi-\frac{1}{4} \mathcal{M}\left(d^{c} \tilde{\omega}\right) \otimes \varphi \\
& =N \otimes \varphi-\alpha \wedge \varphi
\end{aligned}
$$

Consequently (6.42) can be simplified to

$$
N \otimes \varphi=\beta \wedge \hat{\varphi} .
$$

By Lemma 14, the LHS of the above equation is a $(2,2)$-form while the RHS is a $(3,1)+$ $(1,3)$-form. Therefore we conclude that $N \otimes \varphi=0$ and $\beta=0$. As a result,

$$
\tilde{\mathfrak{D}} \Omega=\alpha \otimes \Omega,
$$

which implies immediately that $\tilde{\mathfrak{D}}\left(\frac{\Omega}{|\Omega|_{\tilde{g}}}\right)=0$. Q.E.D.

Remark: Heuristically we can argue as follows. Since $N$ accounts for the non-integrability of $J$, the form $N \otimes \varphi$ is responsible for the "exotic" component of $d \varphi$ which vanishes automatically in the integrable case. Because $\varphi$ is a $(3,0)+(0,3)$-form, $d \varphi$ would be a $(3,1)+(1,3)$-form if $J$ is integrable. As a result

$$
N \otimes \varphi=(2,2) \text { component of } d \varphi=0 .
$$

\section{Corollary 1}

The pair $(N, \varphi)$ satisfies

$$
\begin{aligned}
& N^{p}{ }_{i j} \varphi_{p k l}+N_{k l}^{p} \varphi_{p i j}=0, \\
& N^{p}{ }_{i j} \hat{\varphi}_{p k l}-N_{k l}^{p} \hat{\varphi}_{p i j}=0 .
\end{aligned}
$$

Proof: In the proof of Theorem (3) Part (a), we showed that $N \otimes \varphi=0$, namely

$$
N^{p}{ }_{i j} \varphi_{p k l}+N_{k l}^{p} \varphi_{p i j}-N^{p}{ }_{i k} \varphi_{p j l}-N^{p}{ }_{j l} \varphi_{p i k}+N^{p}{ }_{i l} \varphi_{p j k}+N^{p}{ }_{j k} \varphi_{p i l}=0 .
$$

Replace $i$ and $j$ in (6.47) by $J i$ and $J j$, by using symmetry of $N$ and $\varphi$, we get instead

$$
-N^{p}{ }_{i j} \varphi_{p k l}-N_{k l}^{p} \varphi_{p i j}-N^{p}{ }_{i k} \varphi_{p j l}-N^{p}{ }_{j l} \varphi_{p i k}+N^{p}{ }_{i l} \varphi_{p j k}+N^{p}{ }_{j k} \varphi_{p i l}=0 .
$$

By combining (6.47) and (6.48) we prove the corollary. Equation (6.46) follows from (6.45) and Lemma 6. Q.E.D. 


\subsection{The curvature in Type IIA geometry}

Next, we prove Theorem 3 (b).

As we have seen in Theorem 3 (a), in Type IIA geometry, the nowhere vanishing $(3,0)$ form $\Omega /|\Omega|_{\tilde{g}}$ is parallel under the connection $\tilde{\mathfrak{D}}$. A direct consequence is that the first Chern form associated to $\tilde{\mathfrak{D}}$ is identically zero, that is,

$$
-\frac{\sqrt{-1}}{2} \tilde{\mathfrak{R}}_{i j k l} \tilde{\omega}^{l k}=\tilde{\mathfrak{R}}_{i j}{ }^{\beta}{ }_{\beta}=0 .
$$

As $\tilde{g}=|\varphi|^{2} g$, one can relate $\tilde{\mathfrak{D}}$ with $\mathfrak{D}$ by the conformal change formula. Combining it with (6.44), it is not hard to see that

$$
\mathfrak{D} \Omega=-\frac{1}{2}(\alpha-\sqrt{-1} J \alpha) \otimes \Omega
$$

As a consequence, the curvature tensor $\mathfrak{R}$ satisfies

$$
-\frac{\sqrt{-1}}{2} \mathfrak{R}_{i j k l} \omega^{l k}=\mathfrak{R}_{i j}{ }^{\beta}{ }_{\beta}=\frac{1}{2} d(\alpha-\sqrt{-1} J \alpha)_{i j}=-\sqrt{-1}\left(d d^{c} \log |\varphi|\right)_{i j},
$$

and we recover the well-known formula for Ricci curvature in the Kähler case. In fact, (6.50) implies that $\mathfrak{D}^{0,1} \Omega=0$. For an almost Kähler manifold, the Gauduchon line of connections [26] collapses to a point, so $\mathfrak{D}$ is also the Chern connection (in the almost complex setting), hence $\mathfrak{D}^{0,1}=\bar{\partial}$, and we conclude that $\Omega$ is a holomorphic section of the canonical bundle associated to $(M, J)$. Theorem $3(\mathrm{~b})$ is proved.

We complete this section with some identities linking the curvature and Nijenhuis tensor. Recall the globally defined function $u=\log |\varphi|^{2}$. In this notation we have $\tilde{g}=e^{u} g$ and $\alpha=-d u$. Furthermore (6.51) can be rewritten as

$$
\begin{aligned}
\mathfrak{R}_{i j k l} \omega^{l k} & =\left(d d^{c} u\right)_{i j}=-\left(\mathfrak{D}_{i}(d u)_{J j}-\mathfrak{D}_{j}(d u)_{J i}+N^{k}{ }_{i j} u_{J k}\right) \\
& =-\left(\nabla^{2} u\right)_{i, J j}+\left(\nabla^{2} u\right)_{j, J i}-2 N^{k}{ }_{i j} u_{J k} .
\end{aligned}
$$

Substitute (6.52) back to (6.18), we get

$$
R_{i j}=2 \mathfrak{D}^{k} N_{i j k}-2\left(N_{-}^{2}\right)_{i j}+\frac{1}{2}\left(\nabla^{2} u\right)_{i j}+\frac{1}{2}\left(\nabla^{2} u\right)_{J i, J j}-u_{k} N^{k}{ }_{i j} .
$$

Since $R_{i j}$ is symmetric, we conclude

$$
\begin{aligned}
R_{i j} & =\mathfrak{D}^{k}\left(N_{i j k}+N_{j i k}\right)-2\left(N_{-}^{2}\right)_{i j}+\frac{1}{2}\left(\nabla^{2} u\right)_{i j}+\frac{1}{2}\left(\nabla^{2} u\right)_{J i, J j}, \\
& =\nabla^{k}\left(N_{i j k}+N_{j i k}\right)+2\left(N_{-}^{2}\right)_{i j}-2\left(N_{+}^{2}\right)_{i j}+\frac{1}{2}\left(\nabla^{2} u\right)_{i j}+\frac{1}{2}\left(\nabla^{2} u\right)_{J i, J j} .
\end{aligned}
$$


and that $N$ satisfies

$$
\mathfrak{D}^{k} N_{k i j}=\nabla^{k} N_{k i j}=-u^{k} N_{k i j}
$$

Therefore the $J$-invariant and $J$-anti-invariant components of the Ricci curvature are given by (6.21) and the following refinement of (6.20)

$$
\left(R^{J}\right)_{i j}=-2\left(N_{-}^{2}\right)_{i j}+\left(\left(\nabla^{2} u\right)^{J}\right)_{i j}, \quad\left(R^{-J}\right)_{i j}=\mathfrak{D}^{k}\left(N_{i j k}+N_{j i k}\right) .
$$

The scalar curvature is

$$
R=\Delta u-|N|^{2}
$$

(6.52) also implies that

$$
\frac{1}{2} \Re_{i j k l} \omega^{j i} \omega^{l k}=\Delta u
$$

It follows from (6.22) that the $\star$-scalar curvature is given by

$$
\frac{1}{2} R_{i j k l} \omega^{j i} \omega^{l k}=\Delta u+|N|^{2}
$$

Similarly we can derive the formulae for $\tilde{R}$, the curvature tensor associated to the conformal metric $\tilde{g}$ :

$$
\begin{aligned}
\tilde{R}_{i j}= & -\left(\tilde{\mathfrak{D}}^{s}-\frac{1}{2} u^{s}\right)\left(N_{i s j}+N_{j s i}\right)+\frac{1}{2}\left(\left(\tilde{\nabla}^{2} u\right)_{J i, J j}-3\left(\tilde{\nabla}^{2} u\right)_{i j}-\tilde{\Delta} u \tilde{g}_{i j}\right) \\
& -2\left(N_{-}^{2}\right)_{i j}-\frac{1}{2} u_{i} u_{j}+\frac{1}{2} u_{J i} u_{J j}+\frac{1}{2}|d u|_{\tilde{g}}^{2} \tilde{g}_{i j}, \\
\tilde{R}= & -4 \tilde{\Delta} u+3|d u|_{\tilde{g}}^{2}-|N|_{\tilde{g}}^{2},
\end{aligned}
$$

with (6.55) becoming

$$
2 \tilde{\mathfrak{D}}^{k} N_{k i j}=u^{k} N_{k i j}, \quad 2 \tilde{\nabla}^{k} N_{k i j}=3 u^{k} N_{k i j} .
$$

\subsection{The Nijenhuis tensor in Type IIA geometry}

As we have seen in previous sections, on an almost Kähler manifold, the Nijenhuis tensor $N$ is a $(0,2)$-type $T M$-valued 2-form satisfying the Bianchi identity (3.8). Moreover, one can define two $J$-invariant symmetric tensors $N_{+}^{2}$ and $N_{-}^{2}$ satisfying

$$
\operatorname{tr} N_{+}^{2}=|N|^{2}=2 \operatorname{tr} N_{-}^{2} .
$$

When an almost Kähler structure is enhanced to a Type IIA structure, the integrability of $J$ is improved, hence one should expect more identities satisfied by $N$. For example, 
we have already seen that certain divergences of $N$ are actually terms of lower order term (6.55). In this subsection, we shall derive more identities and differential equations satisfied by $N$, showing that an almost-complex structure coming from a Type IIA geometry is more "integrable" than a generic one. We shall also complete the proof of Theorem 3 by proving Part (c).

First, we show that $N_{+}^{2}$ and $N_{-}^{2}$ are related to each other:

\section{Proposition 1}

For any Type IIA structure $(M, \omega, \varphi)$, the Nijenhuis tensor $N$ satisfies

$$
N_{-}^{2}=2 N_{+}^{2}-\frac{1}{4}|N|^{2} g
$$

Proof: In view of (6.45), we notice that

$$
\varphi_{i a p} N_{b}^{s t} N_{s t}^{p}=-\varphi_{p s t} N_{b}^{s t} N_{i a}^{p}=\varphi_{s t b} N_{p}^{s t} N_{i a}^{p},
$$

so

$$
|\varphi|^{-2} N^{s t}{ }_{b} N^{p}{ }_{s t} \varphi_{i a p} \varphi_{j c d} \omega^{a c} \omega^{b d}=|\varphi|^{-2} N^{s t}{ }_{p} N^{p}{ }_{i a} \varphi_{s t b} \varphi_{j c d} \omega^{a c} \omega^{b d} .
$$

Apply Lemma 8 to both sides, we get

$$
2 N^{s t}{ }_{i} N_{j s t}-N^{s t k} N_{k s t} g_{i j}=2 N_{j}{ }^{s t} N_{t s i}+2 N^{s t}{ }_{j} N_{t s i} .
$$

Converting everything into $N_{+}^{2}$ and $N_{-}^{2}$, we get

$$
-2 N_{+}^{2}+2 N_{-}^{2}-N^{s t k} N_{k s t} g=2 N_{+}^{2} .
$$

By Bianchi identity, we know that

$$
-N^{s t k} N_{k s t}=N^{s t k}\left(N_{s t k}-N_{t s k}\right)=\operatorname{tr} N_{+}^{2}-\operatorname{tr} N_{-}^{2}=\frac{1}{2}|N|^{2} .
$$

Consequently

$$
N_{-}^{2}=2 N_{+}^{2}-\frac{1}{4}|N|^{2} g .
$$

As a corollary, we obtain the inequality

$$
0 \leq N_{+}^{2} \leq \frac{1}{4}|N|^{2} g
$$

since $0 \leq N_{+}^{2}-N_{-}^{2}$. Q.E.D.

We now come to the proof of Theorem 3 (c), which is a very powerful tool for proving identities involving $N$ : 
Let us choose a frame at a given point as in Lemma 4. Since $N$ is a $(0,2)$-type $T M$ valued 2-form satisfying the Bianchi identity, we get the following relations:

$$
\begin{aligned}
& 0=N_{* j j}=N_{* 12}=N_{* 21}=N_{* 34}=N_{* 43}=N_{* 56}=N_{* 65}, \\
& N_{135}=-N_{153}=-N_{146}=N_{164}=-N_{236}=N_{263}=-N_{245}=N_{254}, \\
& N_{136}=-N_{163}=N_{145}=-N_{154}=N_{235}=-N_{253}=-N_{246}=N_{264}, \\
& N_{315}=-N_{351}=-N_{326}=N_{362}=-N_{416}=N_{461}=-N_{425}=N_{452}, \\
& N_{316}=-N_{361}=N_{325}=-N_{352}=N_{415}=-N_{451}=-N_{426}=N_{462}, \\
& N_{513}=-N_{531}=-N_{524}=N_{542}=-N_{614}=N_{641}=-N_{623}=N_{632}, \\
& N_{514}=-N_{541}=N_{523}=-N_{532}=N_{613}=-N_{631}=-N_{624}=N_{642},
\end{aligned}
$$

and

$$
\begin{aligned}
& N_{113}=-N_{131}=-N_{124}=N_{142}=-N_{214}=N_{241}=-N_{223}=N_{232}, \\
& N_{114}=-N_{141}=N_{123}=-N_{132}=N_{213}=-N_{231}=-N_{224}=N_{242}, \\
& N_{115}=-N_{151}=-N_{126}=N_{162}=-N_{216}=N_{261}=-N_{225}=N_{252}, \\
& N_{116}=-N_{161}=N_{125}=-N_{152}=N_{215}=-N_{251}=-N_{226}=N_{262}, \\
& N_{331}=-N_{313}=-N_{342}=N_{324}=-N_{432}=N_{423}=-N_{441}=N_{414}, \\
& N_{332}=-N_{323}=N_{341}=-N_{314}=N_{431}=-N_{413}=-N_{442}=N_{424}, \\
& N_{335}=-N_{353}=-N_{346}=N_{364}=-N_{436}=N_{463}=-N_{445}=N_{454}, \\
& N_{336}=-N_{363}=N_{345}=-N_{354}=N_{435}=-N_{453}=-N_{446}=N_{464}, \\
& N_{551}=-N_{515}=-N_{562}=N_{526}=-N_{652}=N_{625}=-N_{661}=N_{616}, \\
& N_{552}=-N_{525}=N_{561}=-N_{516}=N_{651}=-N_{615}=-N_{662}=N_{626}, \\
& N_{553}=-N_{535}=-N_{564}=N_{546}=-N_{654}=N_{645}=-N_{663}=N_{636}, \\
& N_{554}=-N_{545}=N_{563}=-N_{536}=N_{653}=-N_{635}=-N_{664}=N_{646},
\end{aligned}
$$

with constraints

$$
\begin{aligned}
& N_{135}+N_{351}+N_{513}=0, \\
& N_{136}+N_{361}+N_{613}=0 .
\end{aligned}
$$

Furthermore, by evaluating (6.45) at the given point, we get

$$
\begin{aligned}
& 0=N_{135}=N_{136}=N_{315}=N_{316}=N_{513}=N_{514}, \\
& 0=N_{331}-N_{551}=N_{113}-N_{553}=N_{115}-N_{335}, \\
& 0=N_{114}+N_{554}=N_{116}+N_{336}=N_{332}+N_{552},
\end{aligned}
$$

Therefore $N$ has only 6 independent components at the given point. Q.E.D. 
We can choose and name such independent components as

$$
a:=N_{331}, \quad b:=N_{332}, \quad c:=N_{113}, \quad d:=N_{114}, \quad e:=N_{115}, \quad f:=N_{116} .
$$

It follows that

$$
|N|^{2}=16\left(a^{2}+b^{2}+c^{2}+d^{2}+e^{2}+f^{2}\right) .
$$

We can further express $N_{+}^{2}$ and $N_{-}^{2}$ in terms of these components. For instance, it is straightforward to verify that

$$
N_{+}^{2}=2\left[\begin{array}{cccccc}
r^{2}+a^{2}+b^{2} & 0 & a c+b d & -a d+b c & a e-b f & -a f-b e \\
0 & r^{2}+a^{2}+b^{2} & a d-b c & a c+b d & a f+b e & a e-b f \\
a c+b d & a d-b c & r^{2}+c^{2}+d^{2} & 0 & c e+d f & c f-d e \\
-a d+b c & a c+b d & 0 & r^{2}+c^{2}+d^{2} & -c f+d e & c e+d f \\
a e-b f & a f+b e & c e+d f & -c f+d e & r^{2}+e^{2}+f^{2} & 0 \\
-a f-b e & a e-b f & c f-d e & c e+d f & 0 & r^{2}+e^{2}+f^{2}
\end{array}\right],
$$

where $r^{2}=a^{2}+b^{2}+c^{2}+d^{2}+e^{2}+f^{2}=\frac{1}{16}|N|^{2}$. Similarly we can find $N_{-}^{2}$ as well. This normal form also allows us to quickly prove that

$$
\left|N_{+}^{2}\right|^{2}=48 r^{4}=\frac{3}{16}|N|^{4} .
$$

As an application of Theorem 3 (c), we prove that $N$ satisfies the following differential equation:

\section{Lemma 16}

Given a Type IIA structure $(M, \omega, \varphi)$, the Nijenhuis tensor $N$ satisfies

$$
8 N^{s t i} \nabla_{i} N_{s t j}=8 N^{s t i} \mathfrak{D}_{i} N_{s t j}=\mathfrak{D}_{j}|N|^{2}+u_{j}|N|^{2} .
$$

Proof: By (6.25), we have

$$
\begin{aligned}
& \mathfrak{D}_{i} N_{s j t}-\mathfrak{D}_{s} N_{i j t}-\mathfrak{D}_{j} N_{t i s}+\mathfrak{D}_{t} N_{j i s} \\
= & \mathfrak{D}_{J i} N_{J s, j, t}-\mathfrak{D}_{J s} N_{J i, j, t}-\mathfrak{D}_{J j} N_{J t, i, s}+\mathfrak{D}_{J t} N_{J j, i, s} .
\end{aligned}
$$

Contracting this equation with $N^{s t i}$, we get

$$
2 N^{s t i}\left(\mathfrak{D}_{i} N_{s j t}-\mathfrak{D}_{s} N_{i j t}+\mathfrak{D}_{t} N_{j i s}\right)=N^{s t i}\left(\mathfrak{D}_{j} N_{t i s}-\mathfrak{D}_{J j} N_{J t, i, s}\right) .
$$

The LHS can be simplified as follows

$$
\begin{aligned}
\text { LHS } & =-2 N^{s t i} \mathfrak{D}_{i} N_{s t j}+2 N^{i t s} \mathfrak{D}_{i} N_{s t j}+2 N^{s i t} \mathfrak{D}_{i} N_{j t s} \\
& =2 N^{t i s}\left(\mathfrak{D}_{i} N_{s t j}+\mathfrak{D}_{i} N_{j s t}\right) \\
& =-2 N^{s t i} \mathfrak{D}_{i} N_{s t j} .
\end{aligned}
$$


On the other hand, we see that

$$
N^{s t i} \mathfrak{D}_{j} N_{t i s}=-N^{s t i} \mathfrak{D}_{j} N_{t s i}=-\frac{1}{2} \mathfrak{D}_{j}\left(N^{s t i} N_{t s i}\right)=-\frac{1}{4} \mathfrak{D}_{j}|N|^{2} .
$$

Therefore to prove the lemma, we only need to show that

$$
N^{s t i} \mathfrak{D}_{J j} N_{J t, i, s}=\frac{1}{4} u_{j}|N|^{2},
$$

or equivalently

$$
N^{s t i} \mathfrak{D}_{j} N_{t, s, J i}=\frac{1}{4} u_{J j}|N|^{2} .
$$

We only need to verify (6.65) pointwise. To do so, at any given point, we expand the LHS of (6.65) using the normal form of $\varphi$ in Lemma 4. For simplicity of notation, let us write $B=\mathfrak{D}_{j} N$. Clearly $B$ has the same symmetry as $N$, namely it is a $T M$-valued type $(0,2)$-form and it satisfies the Bianchi identity. By Lemma 4, we get

$$
\begin{aligned}
\text { LHS }= & \sum_{s, t, i} N_{s t i}\left(\mathfrak{D}_{j} N\right)_{t, s, J i} \\
= & N_{331}\left(B_{332}-B_{134}+B_{431}-B_{233}+B_{341}-B_{244}-B_{442}-B_{143}\right) \\
& +N_{331}\left(B_{552}-B_{156}+B_{651}-B_{255}+B_{561}+B_{266}-B_{662}-B_{165}\right) \\
& +N_{332}\left(-B_{331}-B_{234}+B_{432}+B_{133}+B_{342}-B_{144}+B_{441}-B_{243}\right) \\
& -N_{332}\left(-B_{551}-B_{256}+B_{652}+B_{155}+B_{562}-B_{166}+B_{661}-B_{265}\right) \\
& +\ldots \\
= & 4 N_{331}\left(B_{332}+B_{552}\right)+4 N_{113}\left(B_{114}+B_{554}\right)+4 N_{115}\left(B_{116}+B_{336}\right) \\
& +4 N_{332}\left(B_{551}-B_{331}\right)+4 N_{114}\left(B_{553}-B_{113}\right)+4 N_{116}\left(B_{335}-B_{115}\right) .
\end{aligned}
$$

Since

$$
\mathfrak{D}_{j} \varphi=\frac{1}{2}\left(u_{j} \varphi+u_{J j} \hat{\varphi}\right),
$$

by taking derivative of Lemma 6.45, we get

$$
\mathfrak{D}_{j} N^{p}{ }_{a b} \varphi_{p c d}+\mathfrak{D}_{j} N^{p}{ }_{c d} \varphi_{p a b}=-u_{J j} N^{p}{ }_{a b} \hat{\varphi}_{p c d},
$$

or equivalently

$$
B^{p}{ }_{a b} \varphi_{p c d}+B^{p}{ }_{c d} \varphi_{p a b}=-u_{J j} N^{p}{ }_{a b} \hat{\varphi}_{p c d} .
$$

Evaluating the above equation at the given point using Lemma 4, we get the following relations

$$
\begin{array}{ll}
B_{331}-B_{551}=-u_{J j} N_{332}, & B_{332}+B_{552}=u_{J j} N_{331}, \\
B_{113}-B_{553}=-u_{J j} N_{114}, & B_{114}+B_{554}=u_{J j} N_{113}, \\
B_{115}-B_{335}=-u_{J j} N_{116}, & B_{116}+B_{336}=u_{J j} N_{115} .
\end{array}
$$


It follows that

$$
\begin{aligned}
\text { LHS of }(\underline{6.65)} & =4 u_{J j}\left(N_{331}^{2}+N_{332}^{2}+N_{113}^{2}+N_{114}^{2}+N_{115}^{2}+N_{116}^{2}\right) \\
& =\frac{1}{4} u_{J j}|N|^{2} .
\end{aligned}
$$

Q.E.D.

\section{The flow of the metric in the Type IIA flow}

The main task of this section is to prove Theorem 4, which gives explicit formulas for the flows of $\varphi$ and $\tilde{g}_{\varphi}$ in terms of the curvature and Nijenhuis tensors.

\subsection{A tensor coefficients ODE for $\varphi$ : proof of Theorem 4(a)}

We begin with the proof of Theorem 4 (a), which gives the flow of $\varphi$. Since we will be mainly working with the metric $\tilde{g}$, we shall use $\tilde{g}$ to raise or lower indices in this subsection.

It is clear that $\varphi(t)$ is closed and primitive for any $t$. We can also assume that $\varphi$ is positive, since this is an open condition and later estimates (7.25) will show that this property is preserved along the flow. Therefore we get a family of Type IIA structures $(M, \omega, \varphi(t))$. So we can apply formulae in Type IIA geometry to expand the right hand side of the flow equation. Now the right hand side is given by $d \Lambda d\left(|\varphi|^{2} \hat{\varphi}\right)=d \Lambda d\left(e^{u} \hat{\varphi}\right)$. Combining (6.40), (6.30), and $\beta=0$, we obtain

$$
d \hat{\varphi}=\alpha \wedge \hat{\varphi}+\tilde{\mathfrak{T}} \otimes \hat{\varphi}
$$

where $\tilde{\mathfrak{T}}=N-\frac{1}{4}\left(d^{c} \tilde{\omega}+\mathcal{M}\left(d^{c} \tilde{\omega}\right)\right)$ by (6.43). Applying Lemma 15, we obtain $d \hat{\varphi}=N \otimes \hat{\varphi}$ and

$$
d\left(e^{u} \hat{\varphi}\right)=e^{u}(d u \wedge \hat{\varphi}+N \bigotimes \hat{\varphi}) .
$$

To proceed, we need to compute $\Lambda(d u \wedge \hat{\varphi})$ and $\Lambda(N \otimes \hat{\varphi})$, which are 2-forms of type $(2,0)+(0,2)$ and of type $(1,1)$ respectively. We have the following lemmas:

Lemma 17

$$
(\Lambda(d u \wedge \hat{\varphi}))_{k l}=g^{j i} u_{i} \varphi_{j k l}
$$

Proof: Since $(d u \wedge \hat{\varphi})_{i j k l}=u_{i} \hat{\varphi}_{j k l}-u_{j} \hat{\varphi}_{i k l}+u_{k} \hat{\varphi}_{i j l}-u_{l} \varphi_{i j k}$, by definition of $\Lambda$, we have

$$
(\Lambda(d u \wedge \hat{\varphi}))_{k l}=\frac{1}{2} \omega^{j i}\left(u_{i} \hat{\varphi}_{j k l}-u_{j} \hat{\varphi}_{i k l}+u_{k} \hat{\varphi}_{i j l}-u_{l} \hat{\varphi}_{i j k}\right)
$$


The last two terms in the above expression are zero since $\hat{\varphi}$ is primitive. In addition, the first two terms are identical due to the symmetry in switching $i$ and $j$, so it follows that

$$
\begin{aligned}
(\Lambda(d u \wedge \hat{\varphi}))_{k l} & =\omega^{j i} u_{i} \hat{\varphi}_{j k l}=g^{J j, i} u_{i} \hat{\varphi}_{j k l}=g^{j i} u_{i} \hat{\varphi}_{J j, k, l} \\
& =g^{j i} u_{i} \varphi_{j k l},
\end{aligned}
$$

where in the last step we make use of Lemma 6. Q.E.D.

\section{Lemma 18}

$$
\Lambda(N \otimes \hat{\varphi})_{k l}=2 g^{j i} N_{i l}^{p} \varphi_{p j k}=-2 g^{j i} N_{i k}^{p} \varphi_{p j l}
$$

Proof: By (6.31) and (6.46) we see that

$$
\Lambda(N \otimes \hat{\varphi})_{k l}=\omega^{j i}\left(N_{k l}^{p} \hat{\varphi}_{p i j}-N_{i k}^{p} \hat{\varphi}_{p j l}+N_{i l}^{p} \hat{\varphi}_{p j k}\right) .
$$

Notice that the first term above vanishes due to the primitiveness of $\hat{\varphi}$, and again, the last two terms are identical because of the symmetry of switching $i$ and $j$, so we conclude that

$$
\Lambda(N \otimes \hat{\varphi})_{k l}=2 \omega^{j i} N^{p}{ }_{i l} \hat{\varphi}_{p j k}=-2 \omega^{j i} N^{p}{ }_{i l} \varphi_{p, J j, k}=2 g^{j i} N^{p}{ }_{i l} \varphi_{p j k} .
$$

Here we again used Lemma 6 to simplify our expression. Q.E.D.

Combining Lemma 17 and Lemma 18, we see immediately that

$$
\begin{aligned}
\mu_{k l} & :=\left(\Lambda d\left(e^{u} \hat{\varphi}\right)\right)_{k l}=e^{u} g^{j i}\left(u_{i} \varphi_{j k l}+2 N^{p}{ }_{i l} \varphi_{p j k}\right) \\
& =e^{2 u}\left(u^{s} \varphi_{s k l}+2 N^{s t}{ }_{l} \varphi_{s t k}\right)=e^{2 u}\left(u^{s} \varphi_{s k l}-2 N^{s t}{ }_{k} \varphi_{s t l}\right) .
\end{aligned}
$$

To compute $d \Lambda d\left(e^{u} \hat{\varphi}\right)=d \mu$, we make use of Lemma 13 to get

$$
(d \mu)_{i a b}=(\tilde{\mathfrak{T}} \otimes \mu)_{i a b}+\sum_{\text {cyc } i, a, b} \tilde{\mathfrak{D}}_{i} \mu_{a b}
$$

The first term in (7.5) is already in good shape, since by (6.32) we get

$$
\begin{aligned}
(\tilde{\mathfrak{T}} \otimes \mu)_{i a b} & =\sum_{\text {cyc } i, a, b} \tilde{\mathfrak{T}}^{p}{ }_{i a} \mu_{p b}=e^{2 u} \sum_{\text {cyc } i, a, b} \tilde{\mathfrak{T}}^{p}{ }_{i a}\left(u^{s} \varphi_{s p b}-2 N^{s t}{ }_{p} \varphi_{s t b}\right) \\
& =e^{2 u} \sum_{\text {cyc } i, a, b} \varphi_{\text {sta }}\left(2 N^{s t}{ }_{p} \tilde{\mathfrak{T}}^{p}{ }_{i b}-u^{s} \tilde{\mathfrak{T}}^{t}{ }_{i b}\right),
\end{aligned}
$$

which is linear in $\varphi$. For the second term in (7.5), we need

\section{Lemma 19}

$$
\tilde{\mathfrak{D}}_{i} \mu_{a b}=e^{2 u}\left(\varphi_{s a b}\left(\tilde{\mathfrak{D}}_{i}+u_{i}\right) u^{s}+2 \varphi_{s t a}\left(\tilde{\mathfrak{D}}_{i}+u_{i}\right) N_{b}^{s t}\right) .
$$


Proof: Plugging in (7.4), we see that

$$
\begin{aligned}
& \tilde{\mathfrak{D}}_{i} \mu_{a b}=\tilde{\mathfrak{D}}_{i}\left(e^{2 u}\left(u^{s} \varphi_{s a b}+2 N^{s t}{ }_{b} \varphi_{s t a}\right)\right) \\
& =e^{2 u}\left(2 u_{i}\left(u^{s} \varphi_{s a b}+2 N^{s t}{ }_{b} \varphi_{s t a}\right)+u^{s} \tilde{\mathfrak{D}}_{i} \varphi_{s a b}+2 N^{s t}{ }_{b} \tilde{\mathfrak{D}}_{i} \varphi_{s t a}\right. \\
& \left.+\varphi_{s a b} \tilde{\mathfrak{D}}_{i} u^{s}+2 \varphi_{s t a} \tilde{\mathfrak{D}}_{i} N^{s t}{ }_{b}\right) \\
& \stackrel{(6.44)}{=} e^{2 u}\left(\varphi_{s a b}\left(\tilde{\mathfrak{D}}_{i}+u_{i}\right) u^{s}+2 \varphi_{s t a}\left(\tilde{\mathfrak{D}}_{i}+u_{i}\right) N^{s t}{ }_{b}\right) \text {. }
\end{aligned}
$$

Q.E.D.

Combining (7.6) and (7.7) we obtain the evolution equation for $\varphi$ stated in Theorem 4 (a).

Next we justify the remark made after Theorem [4, to the effect that the function $u$ is determined by $\tilde{g}$. Indeed $\tilde{g}=e^{u} g$, and thus To prove the second part of the statement, we notice that $\tilde{g}=e^{u} g$, therefore the volume element associated to $\tilde{g}$ satisfies

$$
\operatorname{dvol}_{\tilde{g}}=e^{3 u} \operatorname{dvol}_{g}=e^{3 u} \frac{\omega^{3}}{3 !} .
$$

Therefore (in Darboux coordinate) we may write the global function $u$ as

$$
u=\frac{1}{6} \log \operatorname{det} \tilde{g},
$$

which is entirely determined by $\tilde{g}$. Hence the metric $g$ is also determined by $\tilde{g}$, and so is the almost complex structurer $J$ since $\omega$ is fixed. It follows that the Nijenhuis tensor $N$, the projected Levi-Civita connection $\tilde{\mathfrak{D}}$ and its torsion $\tilde{\mathfrak{T}}$ are also determined by $\tilde{g}$.

For the convenience of later calculations, we derive a more explicit evolution equation for $\varphi$ than what we have in Theorem 4 Part (a). The starting point is (7.5), which can be expanded as

$$
(d \mu)_{i a b}=(N \otimes \mu)_{i a b}-(U \otimes \mu)_{i a b}+\sum_{\text {cyc } i, a, b} \tilde{\mathfrak{D}}_{i} \mu_{a b}
$$

by using (6.43). The first two terms in (17.9) can be expressed as follows:

\section{Lemma 20}

$$
(N \otimes \mu)_{i a b}=e^{2 u} \sum_{\text {cyc } i, a, b} \varphi_{i a p}\left(u^{s} N^{p}{ }_{s b}-2 N^{s t}{ }_{b} N^{p}{ }_{s t}\right) .
$$

Proof of the Lemma: By (6.32), we have

$$
\begin{aligned}
(N \otimes \mu)_{i a b} & =\sum_{\text {cyc } i, a, b} N^{p}{ }_{i a} \mu_{p b}=e^{2 u} \sum_{\text {cyc } i, a, b} N^{p}{ }_{i a}\left(u^{s} \varphi_{s p b}+2 N^{s t}{ }_{b} \varphi_{s t p}\right) \\
& =e^{2 u} \sum_{\text {cyc } i, a, b} N^{p}{ }_{i a}\left(-u^{s} \varphi_{p s b}+2 N^{s t}{ }_{b} \varphi_{p s t}\right) \\
& \stackrel{6.455}{=} e^{2 u} \sum_{\text {cyc } i, a, b} \varphi_{i a p}\left(u^{s} N^{p}{ }_{s b}-2 N^{s t}{ }_{b} N^{p}{ }_{s t}\right) .
\end{aligned}
$$


Q.E.D.

\section{Lemma 21}

For any real 2 -form $\mu$, we may write $\mu=\mu^{+}+\mu^{-}$, where $\mu^{+}$and $\mu^{-}$are the $(1,1)$ and $(2,0)+(0,2)$ components of $\mu$ respectively. Then

$$
(U \otimes \mu)_{i a b}=-\frac{1}{2} \tilde{\omega}^{q p} u_{q}(\tilde{\omega} \wedge \mu)_{p i a b}-\frac{1}{2} \sum_{\text {cyc } i, a, b} u_{J i}\left(\mu^{-}\right)_{J a, b} .
$$

In particular, for the specific $\mu$ in (7.4), we get

$$
(U \otimes \mu)_{i a b}=\frac{1}{2}\left(\tilde{\omega} \wedge \iota_{W} \mu\right)_{i a b}+\frac{e^{2 u}}{2}|d u|_{\tilde{g}}^{2} \varphi_{i a b}-e^{2 u} \sum_{\text {cyclic } i, a, b} u_{i}\left(u^{s} \varphi_{s a b}+N^{s t}{ }_{b} \varphi_{s t a}\right) .
$$

Proof of the Lemma: By (6.32) and the definition of $U$ (6.28), we have

$$
\begin{aligned}
(U \otimes \mu)_{i a b} & =\tilde{g}^{p q} \sum_{\text {сус } i, a, b} U_{q i a} \mu_{p b} \\
& =\frac{1}{4} \tilde{g}^{p q} \sum_{\text {cyc } i, a, b}\left(2 \alpha_{J q} \tilde{\omega}_{i a}+\alpha_{J i} \tilde{\omega}_{a q}+\alpha_{J a} \tilde{\omega}_{q i}+\alpha_{i} \tilde{g}_{a q}-\alpha_{a} \tilde{g}_{q i}\right) \mu_{p b} \\
& =\frac{1}{4} \sum_{\text {cyc } i, a, b} 2 \tilde{\omega}^{q p} \alpha_{q} \tilde{\omega}_{i a} \mu_{p b}+\alpha_{J i} \mu_{J a, b}-\alpha_{J a} \mu_{J i, b}+\alpha_{i} \mu_{a b}-\alpha_{a} \mu_{i b} \\
& =\frac{1}{2}(\alpha \wedge \mu)_{i a b}+\frac{1}{4} \sum_{\text {cyc } i, a, b} 2 \tilde{\omega}^{q p} \alpha_{q} \tilde{\omega}_{i a} \mu_{p b}+\alpha_{J i}\left(\mu_{J a, b}-\mu_{J b, a}\right) .
\end{aligned}
$$

Notice that $(\tilde{\omega} \wedge \mu)_{p i a b}=\tilde{\omega}_{i a} \mu_{p b}+\tilde{\omega}_{a b} \mu_{p i}+\tilde{\omega}_{b i} \mu_{p a}-\tilde{\omega}_{p a} \mu_{i b}-\tilde{\omega}_{p b} \mu_{a i}-\tilde{\omega}_{p i} \mu_{b a}$, therefore

$$
\tilde{\omega}^{q p} \alpha_{q}(\tilde{\omega} \wedge \mu)_{p i a b}=(\alpha \wedge \mu)_{i a b}+\tilde{\omega}^{q p} \alpha_{q} \sum_{\text {cyc } i, a, b} \tilde{\omega}_{i a} \mu_{p b} .
$$

Hence we find out that

$$
(U \otimes \mu)_{i a b}=\frac{1}{2} \tilde{\omega}^{q p} \alpha_{q}(\tilde{\omega} \wedge \mu)_{p i a b}+\frac{1}{4} \sum_{\text {cyc } i, a, b} \alpha_{J i}\left(\mu_{J a, b}-\mu_{J b, a}\right) .
$$

Write $\mu=\mu^{+}+\mu^{-}$, where $\mu^{+}$and $\mu^{-}$are the $(1,1)$ and $(2,0)+(0,2)$ components. Then by definition, we have

$$
\left(\mu^{+}\right)_{J a, b}=\left(\mu^{+}\right)_{J b, a}, \quad\left(\mu^{-}\right)_{J a, b}=-\left(\mu^{-}\right)_{J b, a} .
$$

So we conclude that

$$
(U \otimes \mu)_{i a b}=-\frac{1}{2} \tilde{\omega}^{q p} u_{q}(\tilde{\omega} \wedge \mu)_{p i a b}-\frac{1}{2} \sum_{\text {cyc } i, a, b} u_{J i}\left(\mu^{-}\right)_{J a, b} .
$$


Now let us apply this to $\mu=\Lambda d\left(e^{u} \hat{\varphi}\right)$. It is clear from (17.4) that

$$
\left(\mu^{-}\right)_{a b}=e^{2 u} u^{s} \varphi_{s a b}=e^{2 u}\left(\iota_{\tilde{\nabla} u} \varphi\right)_{a b} .
$$

Let $W=W^{p} \partial_{p}$ be the vector field defined by $W^{p}=-\tilde{\omega}^{q p} u_{q}=(\tilde{\nabla} u)^{J p}$, we see that

$$
-\frac{1}{2} \tilde{\omega}^{q p} u_{q}(\tilde{\omega} \wedge \mu)_{p i a b}=\frac{1}{2}\left(\iota_{W}(\tilde{\omega} \wedge \mu)\right)_{i a b}=\frac{1}{2}\left(\tilde{\omega} \wedge \iota_{W} \mu\right)_{i a b}-\frac{1}{2}(d u \wedge \mu)_{i a b},
$$

hence

$$
\begin{aligned}
(U \otimes \mu)_{i a b} & \stackrel{(7.13)}{=} \frac{1}{2}\left(\tilde{\omega} \wedge \iota_{W} \mu\right)_{i a b}-\frac{1}{2}(d u \wedge \mu)_{i a b}-\frac{1}{2} e^{2 u} \sum_{\text {cyclic } i, a, b} u_{J i} u^{J s} \varphi_{s a b} \\
\stackrel{(7.4)}{=} & \frac{1}{2}\left(\tilde{\omega} \wedge \iota_{W} \mu\right)_{i a b}-\frac{e^{2 u}}{2}\left(d u \wedge \iota_{\tilde{\nabla} u} \varphi+J d u \wedge \iota_{J \tilde{\nabla} u} \varphi\right)-e^{2 u} \sum_{\text {cyclic } i, a, b} u_{i} N^{s t}{ }_{b} \varphi_{s t a} .
\end{aligned}
$$

From (4.13) we know that

$$
d u \wedge \varphi=-J d u \wedge J \varphi
$$

by taking interior product with $\tilde{\nabla} u$, we get

$$
|d u|_{\tilde{g}}^{2} \varphi-d u \wedge \iota_{\tilde{\nabla} u} \varphi=\iota_{\tilde{\nabla} u}(d u \wedge \varphi)=-\iota_{\tilde{\nabla} u}(J d u \wedge J \varphi)=-J d u \wedge \iota_{J \tilde{\nabla} u} \varphi .
$$

Substitute the RHS of the above equation back to the previous one, we get

$$
(U \otimes \mu)_{i a b}=\frac{1}{2}\left(\tilde{\omega} \wedge \iota_{W} \mu\right)_{i a b}+\frac{e^{2 u}}{2}|d u|_{\tilde{g}}^{2} \varphi_{i a b}-e^{2 u} \sum_{\text {cyclic } i, a, b} u_{i}\left(u^{s} \varphi_{s a b}+N^{s t}{ }_{b} \varphi_{s t a}\right) .
$$

Q.E.D.

Combining (7.10), (7.12) and (7.7), we conclude that

$$
\begin{aligned}
\partial_{t} \varphi_{i a b}= & (d \mu)_{i a b} \\
= & -\frac{1}{2}\left(\tilde{\omega} \wedge \iota_{W} \mu\right)_{i a b}-\frac{e^{2 u}}{2}|d u|_{\tilde{g}}^{2} \varphi_{i a b}+e^{2 u} \sum_{\text {cyc } i, a, b} \varphi_{i a p}\left(u^{s} N^{p}{ }_{s b}-2 N^{s t}{ }_{b} N^{p}{ }_{s t}\right) \\
& +e^{2 u} \sum_{\text {cyc } i, a, b}\left(\varphi_{s a b}\left(\tilde{\mathfrak{D}}_{i}+2 u_{i}\right) u^{s}+\varphi_{s t a}\left(2 \tilde{\mathfrak{D}}_{i}+3 u_{i}\right) N^{s t}{ }_{b}\right) .
\end{aligned}
$$

\subsection{The flow of $\tilde{g}_{\varphi}$ : proof of Theorem 4(b)}

By definition of $\tilde{g}$ (4.12), we know that

$$
\begin{aligned}
\partial_{t} \tilde{g}_{i j} & =-\partial_{t} \varphi_{i a b} \varphi_{j c d} \omega^{a c} \omega^{b d}-\varphi_{i a b} \partial_{t} \varphi_{j c d} \omega^{a c} \omega^{b d} \\
& =-\partial_{t} \varphi_{i a b} \varphi_{j c d} \omega^{a c} \omega^{b d}+(i \leftrightarrow j) .
\end{aligned}
$$

We only need to compute the first term in (7.15) as the full expression is the symmetrization of the first term there. This term can be calculated using (7.14). It is useful to observe the following: 
Lemma 22 Suppose $\lambda$ is a 3-form that can be factorized as the product of a 1-form with $\omega$, i.e. $\lambda=\nu \wedge \omega$ for some 1-form $\nu$. Then

$$
\left(\varphi_{i a b} \lambda_{j c d}+\lambda_{i a b} \varphi_{j c d}\right) \omega^{a c} \omega^{b d}=0 .
$$

Proof of the lemma. By our assumption, $\lambda_{i a b}=\nu_{i} \omega_{a b}+\nu_{a} \omega_{b i}+\nu_{b} \omega_{i a}$. Therefore

$$
\begin{aligned}
\lambda_{i a b} \varphi_{j c d} \omega^{a c} \omega^{b d} & =\left(\nu_{i} \omega_{a b}+\nu_{a} \omega_{b i}+\nu_{b} \omega_{i a}\right) \varphi_{j c d} \omega^{a c} \omega^{b d} \\
& =\nu_{i} \varphi_{j c d} \omega^{d c}-\nu_{a} \varphi_{j c i} \omega^{a c}+\nu_{b} \varphi_{j i d} \omega^{b d} \\
& =-2 \nu_{a} \omega^{a c} \varphi_{i j c}
\end{aligned}
$$

where the primitiveness of $\varphi$ is used. After symmetrization in $i$ and $j$, the outcome is zero. Q.E.D.

By Lemma 22, we do not need to worry about the first term in (7.14). For simplicity of notation, let $F$ be the 3 -form defined by

$$
\begin{aligned}
F_{i a b} & =\sum_{\text {cyc } i, a, b}\left(\varphi_{i a p}\left(u^{s} N^{p}{ }_{s b}-2 N^{s t}{ }_{b} N^{p}{ }_{s t}\right)+\varphi_{s a b}\left(\tilde{\mathfrak{D}}_{i}+2 u_{i}\right) u^{s}+\varphi_{s t a}\left(2 \tilde{\mathfrak{D}}_{i}+3 u_{i}\right) N^{s t}{ }_{b}\right) \\
& =\sum_{\text {cyc } i, a, b}\left(\varphi_{\text {pab }}\left(u^{s} N^{p}{ }_{s i}-2 N^{s t}{ }_{i} N^{p}{ }_{s t}+\left(\tilde{\mathfrak{D}}_{i}+2 u_{i}\right) u^{p}\right)+\varphi_{s t a}\left(2 \tilde{\mathfrak{D}}_{i}+3 u_{i}\right) N^{s t}{ }_{b}\right), \quad(7 .
\end{aligned}
$$

where (7.14) can be rewritten as

$$
\partial_{t} \varphi=-\frac{1}{2} \tilde{\omega} \wedge \iota_{W} \mu-\frac{e^{2 u}}{2}|d u|_{\tilde{g}}^{2} \varphi+e^{2 u} F
$$

and we have that

$$
e^{-2 u} \partial_{t} \tilde{g}_{i j}+|d u|_{\tilde{g}}^{2} \tilde{g}_{i j}=-F_{i a b} \varphi_{j c d} \omega^{a c} \omega^{b d}+(i \leftrightarrow j) .
$$

The goal is to compute $F_{i a b} \varphi_{j c d} \omega^{a c} \omega^{b d}$. By (7.16) we know

$$
\begin{aligned}
& F_{i a b} \varphi_{j c d} \omega^{a c} \omega^{b d} \\
= & \varphi_{j c d} \omega^{a c} \omega^{b d} \sum_{\text {cyc } i, a, b}\left(\varphi_{p a b}\left(u^{s} N^{p}{ }_{s i}-2 N^{s t}{ }_{i} N^{p}{ }_{s t}+\left(\tilde{\mathfrak{D}}_{i}+2 u_{i}\right) u^{p}\right)+\varphi_{s t a}\left(2 \tilde{\mathfrak{D}}_{i}+3 u_{i}\right) N^{s t}{ }_{b}\right) \\
= & \varphi_{p a b} \varphi_{j c d} \omega^{a c} \omega^{b d}\left(u^{s} N^{p}{ }_{s i}-2 N^{s t}{ }_{i} N^{p}{ }_{s t}+\left(\tilde{\mathfrak{D}}_{i}+2 u_{i}\right) u^{p}\right) \\
& +2 \varphi_{i a p} \varphi_{j c d} \omega^{a c} \omega^{b d}\left(u^{s} N^{p}{ }_{s b}-2 N^{s t}{ }_{b} N^{p}{ }_{s t}+\left(\tilde{\mathfrak{D}}_{b}+2 u_{b}\right) u^{p}\right) \\
& \left.+\varphi_{s t b} \varphi_{j c d} \omega^{a c} \omega^{b d}\left(\left(4 \tilde{\mathfrak{D}}_{a}+6 u_{a}\right) N^{s t}{ }_{i}-\left(2 \tilde{\mathfrak{D}}_{i}+3 u_{i}\right) N^{s t}{ }_{a}\right)\right) \\
=: & (A)+(B)+(C),
\end{aligned}
$$

where $(A),(B)$, and $(C)$ denote the first, the second, and the third line in (17.18) respectively. $(A)$ can be computed using definition of $\tilde{g}(4.12)$ directly:

$$
\begin{aligned}
(A) & =-\left(u^{s} N_{j s i}-2 N^{s t}{ }_{i} N_{j s t}+\left(\tilde{\mathfrak{D}}_{i}+2 u_{i}\right) u_{j}\right) \\
& =-u^{s} N_{j s i}+2\left(N_{-}^{2}\right)_{i j}-2\left(N_{+}^{2}\right)_{i j}-\tilde{\mathfrak{D}}_{i} u_{j}-2 u_{i} u_{j} .
\end{aligned}
$$


To compute $(B)$ and $(C)$, we need to invoke Lemma 8, It follows that

$$
\begin{aligned}
(B)= & 2 \varphi_{i a p} \varphi_{j c d} \omega^{a c} \omega^{b d}\left(u^{s} N^{p}{ }_{s b}-2 N^{s t}{ }_{b} N^{p}{ }_{s t}+\left(\tilde{\mathfrak{D}}_{b}+2 u_{b}\right) u^{p}\right) \\
= & \frac{1}{2} \omega^{b d}\left(\omega_{i j} \tilde{g}_{p d}-\omega_{p j} \tilde{g}_{i d}-\omega_{i d} \tilde{g}_{p_{j}}+\omega_{p d} \tilde{g}_{i j}\right)\left(u^{s} N^{p}{ }_{s b}-2 N^{s t}{ }_{b} N^{p}{ }_{s t}+\left(\tilde{\mathfrak{D}}_{b}+2 u_{b}\right) u^{p}\right) \\
= & \frac{1}{2}\left(\tilde{\omega}_{i j} J^{b}{ }_{p}-\tilde{\omega}_{p j} J_{i}^{b}+\tilde{g}_{p j} \delta_{i}^{b}-\tilde{g}_{i j} \delta^{b}{ }_{p}\right)\left(u^{s} N^{p}{ }_{s b}-2 N^{s t}{ }_{b} N^{p}{ }_{s t}+\left(\tilde{\mathfrak{D}}_{b}+2 u_{b}\right) u^{p}\right) \\
= & \frac{1}{2}\left(-4 N^{s t}{ }_{i} N_{j s t}+2 N^{s t p} N_{p s t} \tilde{g}_{i j}+\tilde{\omega}_{i j} \tilde{\omega}^{b p} \tilde{\mathfrak{D}}_{b} u_{p}+\left(\tilde{\mathfrak{D}}_{i}+2 u_{i}\right) u_{j}+\left(\tilde{\mathfrak{D}}_{J i}+2 u_{J i}\right) u_{J j}\right. \\
& \left.-\left(\tilde{\mathfrak{D}}^{s} u_{s}+2|d u|_{\tilde{g}}^{2}\right) \tilde{g}_{i j}\right) \\
= & 2\left(N_{+}^{2}\right)_{i j}-2\left(N_{-}^{2}\right)_{i j}+\frac{1}{2}\left(\left(\tilde{\mathfrak{D}}_{i}+2 u_{i}\right) u_{j}+\left(\tilde{\mathfrak{D}}_{J i}+2 u_{J i}\right) u_{J j}+\tilde{\omega}_{i j} \tilde{\omega}^{b p} \tilde{\mathfrak{D}}_{b} u_{p}\right) \\
& -\frac{1}{2}\left(|N|_{\tilde{g}}^{2}+\tilde{\mathfrak{D}}^{s} u_{s}+2|d u|_{\tilde{g}}^{2}\right) \tilde{g}_{i j}
\end{aligned}
$$

and

$$
\begin{aligned}
(C) & \left.=\varphi_{s t b} \varphi_{j c d} \omega^{a c} \omega^{b d}\left(\left(4 \tilde{\mathfrak{D}}_{a}+6 u_{a}\right) N^{s t}{ }_{i}-\left(2 \tilde{\mathfrak{D}}_{i}+3 u_{i}\right) N^{s t}{ }_{a}\right)\right) \\
& \left.=\frac{1}{4} \omega^{a c}\left(\omega_{s j} \tilde{g}_{t c}-\omega_{t j} \tilde{g}_{s c}-\omega_{s c} \tilde{g}_{t j}+\omega_{t c} \tilde{g}_{s j}\right)\left(\left(4 \tilde{\mathfrak{D}}_{a}+6 u_{a}\right) N^{s t}{ }_{i}-\left(2 \tilde{\mathfrak{D}}_{i}+3 u_{i}\right) N^{s t}{ }_{a}\right)\right) \\
& \left.=\frac{1}{4}\left(\tilde{\omega}_{s j} J^{a}{ }_{t}-\tilde{\omega}_{t j} J^{a}{ }_{s}+\tilde{g}_{t j} \delta^{a}{ }_{s}-\tilde{g}_{s j} \delta^{a}{ }_{t}\right)\left(\left(4 \tilde{\mathfrak{D}}_{a}+6 u_{a}\right) N^{s t}{ }_{i}-\left(2 \tilde{\mathfrak{D}}_{i}+3 u_{i}\right) N^{s t}{ }_{a}\right)\right) \\
& =-\left(2 \tilde{\mathfrak{D}}^{s}+3 u^{s}\right) N_{i s j} .
\end{aligned}
$$

Combining (7.19) $,(7.20),(7.21)$, and (17.17), we get

$$
\begin{aligned}
\partial_{t} \tilde{g}_{i j}= & e^{2 u}\left[2\left(\tilde{\mathfrak{D}}^{k}+2 u^{k}\right)\left(N_{i k j}+N_{j k i}\right)+\frac{1}{2}\left(\tilde{\mathfrak{D}}_{i} u_{j}+\tilde{\mathfrak{D}}_{j} u_{i}-\tilde{\mathfrak{D}}_{J i} u_{J j}-\tilde{\mathfrak{D}}_{J j} u_{J i}\right)\right. \\
& \left.+2 u_{i} u_{j}-2 u_{J i} u_{J j}+\left(\tilde{\mathfrak{D}}^{s} u_{s}+|d u|_{\tilde{g}}^{2}+|N|_{\tilde{g}}^{2}\right) \tilde{g}_{i j}\right] .
\end{aligned}
$$

Equation (7.22) is self-contained in the sense that its RHS is entirely determined by the metric $\tilde{g}$ and the $\varphi$-dependence is fully eliminated. However, to study its analytic behavior, we need to rewrite it in a more familiar form as we have in the case of Ricci flow or the LêWang flow [33. Moreover, we would like to replace all the $\tilde{\mathfrak{D}}$-derivatives to $\tilde{\nabla}$-derivatives as it is more convenient for us to apply the conformal change technique.

Notice that

$$
\tilde{\mathfrak{D}}_{i} u_{j}=\tilde{\nabla}_{i} u_{j}-u^{k} N_{i k j}+\frac{1}{4}\left(u_{i} u_{j}+u_{J i} u_{J j}-|d u|_{\tilde{g}}^{2} \tilde{g}_{i j}\right),
$$

therefore (7.22) can be rephrased as

$$
\begin{aligned}
\partial_{t} \tilde{g}_{i j}= & e^{2 u}\left[\left(2 \tilde{\mathfrak{D}}^{k}+3 u^{k}\right)\left(N_{i k j}+N_{j k i}\right)+\left(\tilde{\nabla}^{2} u\right)_{i j}-\left(\tilde{\nabla}^{2} u\right)_{J i, J j}\right. \\
& \left.2 u_{i} u_{j}-2 u_{J i} u_{J j}+\left(\tilde{\Delta} u+|N|_{\tilde{g}}^{2}\right) \tilde{g}_{i j}\right] .
\end{aligned}
$$


Taking (6.59) into account, we obtain the desired formula

$$
\begin{aligned}
\partial_{t} \tilde{g}_{i j}= & e^{2 u}\left[-2 \tilde{R}_{i j}-2\left(\tilde{\nabla}^{2} u\right)_{i j}+4 u^{k}\left(N_{i k j}+N_{j k i}\right)+u_{i} u_{j}-u_{J i} u_{J j}-4\left(N_{-}^{2}\right)_{i j}\right. \\
& \left.+\left(|d u|_{\tilde{g}}^{2}+|N|_{\tilde{g}}^{2}\right) \tilde{g}_{i j}\right] .
\end{aligned}
$$

Q.E.D.

Recalling that $u=(1 / 6) \log \operatorname{det} \tilde{g}$, we can derive from (7.23) that

$$
\begin{aligned}
& \partial_{t} u=\frac{e^{2 u}}{3}\left(-\tilde{\Delta} u-\tilde{R}+3|d u|_{\tilde{g}}^{2}+2|N|_{\tilde{g}}^{2}\right) \\
& \stackrel{(6.60)}{=} \\
& e^{2 u}\left(\tilde{\Delta} u+|N|_{\tilde{g}}^{2}\right) .
\end{aligned}
$$

By the maximum principle, we immediately prove the following estimate

Lemma 23 Suppose $\varphi(t)$ is a solution to the source-free Type IIA flow on $M \times[0, T]$. Then

$$
|\varphi(t)|^{2} \geq \min _{M}\left|\varphi_{0}\right|^{2}
$$

for any $t \in[0, T]$.

This lemma has the important consequence that if $\varphi(t)$ is a solution to the source-free Type IIA flow on $M \times[0, T]$ with primitive closed initial data, then $\varphi(t)$ remains positive on $M \times[0, T]$, which allows us to define the almost-complex structure $J$ and the metric $g$. Indeed, the lemma implies that $\sqrt{-\lambda_{\varphi}}=\frac{1}{2}|\varphi|^{2} \frac{\omega^{3}}{3 !}$ cannot pass through zero.

\subsection{Conformal transformation to a perturbed Ricci flow}

In this subsection, we wish to establish the uniqueness of the flow (7.23). Besides the Ricci curvature, the right hand side of (7.23) also contains the 2 nd order term $\tilde{\nabla}^{2} u$, which cannot be reparametrized away since this would change the symplectic structure and the reparametrized flow would be non-local. Therefore we need a different technique to deal with the Hessian term, namely we absorb it in the Ricci tensor by a conformal change of metric.

More specifically, we consider a family of conformal Hermitian metrics $g^{(s)}=e^{s u} g$, where we have $g^{(0)}=g$ and $g^{(1)}=\tilde{g}$. Notice that all the metrics $g^{(s)}$ are equivalent except for $s=0$, in which case we need the pair $(g, u)$. This is because one can solve $u$ from $g^{(s)}$ when $s \neq 0$ by

$$
u=\frac{1}{6 s} \log \operatorname{det} g^{(s)}
$$


Thus we only need to show the short-time existence and uniqueness of any of the flows satisfied by $g^{(s)}$ with $s \neq 0$, or that for the coupled flow $(g, u)$. To begin with, we first compute the evolution equation satisfied by the pair $(g, u)$.

$$
\begin{aligned}
& \partial_{t} g_{i j} \\
= & \partial_{t}\left(e^{-u} \tilde{g}_{i j}\right)=e^{-u}\left(\partial_{t} \tilde{g}_{i j}-\partial_{t} u \cdot \tilde{g}_{i j}\right) \\
= & e^{u}\left[-2 \tilde{R}_{i j}-2\left(\tilde{\nabla}^{2} u\right)_{i j}+4 u^{k}\left(N_{i k j}+N_{j k i}\right)+u_{i} u_{j}-u_{J i} u_{J j}-4\left(N_{-}^{2}\right)_{i j}\right. \\
& \left.+\left(|d u|_{\tilde{g}}^{2}-\tilde{\Delta} u\right) \tilde{g}_{i j}\right] \\
= & e^{u}\left[-2 R_{i j}+2\left(\nabla^{2} u\right)_{i j}+u_{i} u_{j}-u_{J i} u_{J j}+4 u^{k}\left(N_{i k j}+N_{j k i}\right)-4\left(N_{-}^{2}\right)_{i j}\right] \\
& \stackrel{(\underline{6.53})}{=} \quad 2 e^{u}\left[-\left(R^{-J}\right)_{i j}+\left(\left(\nabla^{2} u\right)^{-J}\right)_{i j}+\left((d u \otimes d u)^{-J}\right)_{i j}+2 u^{s}\left(N_{i s j}+N_{j s i}\right)\right]
\end{aligned}
$$

Meanwhile (7.24) can be rewritten as

$$
\partial_{t} u=e^{u}\left(\Delta u+2|d u|^{2}+|N|^{2}\right)
$$

Using the same method, we can derive that

$$
\begin{aligned}
\partial_{t} g_{i j}^{(s)}= & e^{(s+1) u}\left[-2 R_{i j}^{(s)}+(2-4 s)\left(\left(\nabla^{(s)}\right)^{2} u\right)_{i j}+\left(1+2 s-2 s^{2}\right) u_{i} u_{j}-u_{J i} u_{J j}\right. \\
& \left.+4 u^{k}\left(N_{i k j}+N_{j k i}\right)-4\left(N_{-}^{2}\right)_{i j}+s\left(|d u|_{g^{(s)}}^{2}+|N|_{g^{(s)}}^{2}\right) g_{i j}^{(s)}\right] \\
= & e^{(s+1) u}\left[2\left(\left(-R^{(s)}+(1-2 s)\left(\nabla^{(s)}\right)^{2} u+\left(1+s-s^{2}\right) d u \otimes d u\right)^{-J}\right)_{i j}\right. \\
& \left.+4 u^{k}\left(N_{i k j}+N_{j k i}\right)+s\left(\Delta^{(s)} u+2(1-s)|d u|_{g^{(s)}}^{2}+|N|_{g^{(s)}}^{2}\right) g_{i j}^{(s)}\right] .
\end{aligned}
$$

Formulae (6.54) and (6.55) now take the form

$$
\begin{aligned}
R_{i j}^{(s)}= & -\nabla_{k}^{(s)}\left(N_{i}{ }^{k}{ }_{j}+N_{j}{ }^{k}{ }_{i}\right)+\left(\frac{1}{2}-2 s\right)\left(\left(\nabla^{(s)}\right)^{2} u\right)_{i j}+\frac{1}{2}\left(\left(\nabla^{(s)}\right)^{2} u\right)_{J i, J j}-\frac{s}{2} \Delta^{(s)} u g_{i j}^{(s)} \\
& +\frac{s}{2}(1-2 s) u_{i} u_{j}+\frac{s}{2} u_{J i} u_{J j}-\frac{s}{2}(1-2 s)|d u|_{g^{(s)}}^{2} g_{i j}^{(s)}+\frac{3 s}{2} u^{k}\left(N_{i k j}+N_{j k i}\right) \\
& +2\left(N_{-}^{2}\right)_{i j}-2\left(N_{+}^{2}\right)_{i j}, \\
\nabla_{k}^{(s)} N^{k}{ }_{i j}= & \frac{5 s-2}{2} u^{k} N_{k i j} .
\end{aligned}
$$


In particular for $s=\frac{1}{2}$, from (7.29) we know that the metric $\check{g}:=g^{\left(\frac{1}{2}\right)}$ evolves by

$$
\begin{aligned}
\partial_{t} \check{g}_{i j}= & e^{\frac{3}{2} u}\left[-2 \check{R}_{i j}+\frac{3}{2} u_{i} u_{j}-u_{J i} u_{J j}+4 u^{k}\left(N_{i k j}+N_{j k i}\right)-4\left(N_{-}^{2}\right)_{i j}\right. \\
& \left.+\frac{1}{2}\left(|d u|_{\check{g}}^{2}+|N|_{\check{g}}^{2}\right) \check{g}_{i j}\right],
\end{aligned}
$$

where the only 2nd order term on RHS is the Ricci curvature term. We stress that it is important to keep in mind the fact that $\check{g}_{i j}$ arises from a conformal change from a Type IIA geometry.

\subsection{An integrability condition: proof of Theorem 5}

We now prove Theorem 5, which provides the key integrability condition needed later to establish the uniqueness of the Type IIA solutions to the flow (7.33) of the metrics $\check{g}_{i j}$.

We know that for the Ricci flow, the integrability operator $L$ comes from the contracted Bianchi identity. Since our flow (7.33) can be viewed as a deformation of the Ricci flow, our $L$ should be a deformation of the contracted Bianchi operator. Let us simplify our notation in (7.33) by introducing the tensor $S$ defined as

$$
S_{i j}:=\frac{3}{2} u_{i} u_{j}-u_{J i} u_{J j}+4 u^{k}\left(N_{i k j}+N_{j k i}\right)-4\left(N_{-}^{2}\right)_{i j}+\frac{1}{2}\left(|d u|_{\check{g}}^{2}+|N|_{\check{g}}^{2}\right) \check{g}_{i j}
$$

so (7.33) can be written as

$$
\partial_{t} \check{g}_{i j}=e^{\frac{3}{2} u}\left(-2 \check{R}_{i j}+S_{i j}\right)
$$

where we can think of $S$ as the lower order deformation term of the Ricci curvature. Let $L_{0}$ denote the contracted Bianchi identity operator defined by

$$
L_{0}(P)_{j}:=2 \check{g}^{i k} \check{\nabla}_{k} P_{i j}-\check{g}^{i k} \check{\nabla}_{j} P_{i k}
$$

for any symmetric 2-tensor $P$. We know that $L_{0}\left(-2 \check{R}_{i j}\right)=0$. Now we would like to look for a zeroth order linear operator $Z$ such that $\left(L_{0}+Z\right)\left(-2 \check{R}_{i j}+S_{i j}\right)$ is of degree 1 in the metric $\check{g}$. To do so we need to compute $L_{0}\left(S_{i j}\right)$ first.

\section{Proposition 2}

$$
\begin{aligned}
L_{0}(S)_{j}= & 4 u^{s} \check{R}_{s j}-8 \check{R}_{s k} N^{k s}{ }_{j}-\frac{8}{3} u_{j} \check{R}-16 u^{s}\left(N_{-}^{2}\right)_{s j}+2 u_{s} u_{k} N^{k s}{ }_{j} \\
& +\frac{1}{3} u_{j}\left(2|d u|_{\check{g}}^{2}-5|N|_{\check{g}}^{2}\right) .
\end{aligned}
$$


Proof of the Proposition: We apply $L_{0}$ to each term of $S$ in (7.34) to get

$$
\begin{aligned}
\frac{3}{2} L_{0}\left(u_{i} u_{j}\right) & =3 \check{\Delta} u \cdot u_{j}, \\
-L_{0}\left(u_{J i} u_{J j}\right) & =2 \check{g}^{i k} u_{k}\left(\check{\nabla}^{2} u\right)_{J i, J j}+\check{\nabla}_{j}|d u|_{\check{g}}^{2}-2 \check{g}^{i k} u_{s} u_{t} \check{\nabla}_{i}\left(J^{s}{ }_{j} J^{t}{ }_{k}\right) \\
& =2 \check{g}^{i k} u_{k}\left(\check{\nabla}^{2} u\right)_{J i, J j}+\check{\nabla}_{j}|d u|_{\breve{g}}^{2}+4 u_{s} u_{k} N^{k s}{ }_{j}, \\
4 L_{0}\left(u^{k}\left(N_{i k j}+N_{j k i}\right)\right) & =8\left(\check{\nabla}^{2} u\right)_{s k} N^{k s}{ }_{j}+8 u^{s} \check{\nabla}^{i}\left(N_{i s j}+N_{j s i}\right), \\
-4 L_{0}\left(N_{-}^{2}\right)_{j} & =-8 \check{g}^{i k} \check{\nabla}_{k}\left(N_{-}^{2}\right)_{i j}+2 \check{\nabla}_{j}|N|_{\check{g}}^{2}, \\
\frac{1}{2} L_{0}\left(\left(|d u|_{\check{g}}^{2}+|N|_{\check{g}}^{2}\right) \check{g}\right)_{j} & =-2 \check{\nabla}_{j}|d u|_{\breve{g}}^{2}-2 \check{\nabla}_{j}|N|_{\breve{g}}^{2} .
\end{aligned}
$$

To obtain these expressions, we need to use that

$$
\begin{aligned}
& \nabla_{i} J^{s}{ }_{t}=-2 N_{J i}{ }^{s}{ }^{t}, \\
& \check{\nabla}_{i} J^{s}{ }_{t}=-2 N_{J i}{ }^{s}+\frac{1}{4}\left(u_{J t} \delta^{s}{ }_{i}+g_{i t} u^{J s}-\omega_{i t} u^{s}-u_{t} J^{s}{ }_{i}\right)
\end{aligned}
$$

and we raise and lower indices using the metric $\check{g}$. Combining the calculation above, we get

$$
\begin{aligned}
L_{0}(S)_{j}= & 3 u_{j} \check{\Delta} u+2 u^{i}\left(\check{\nabla}^{2} u\right)_{J i, J j}+8\left(\check{\nabla}^{2} u\right)_{s k} N_{j}^{k s}+8 u^{s} \check{\nabla}^{i}\left(N_{i s j}+N_{j s i}\right) \\
& -8 \check{\nabla}^{i}\left(N_{-}^{2}\right)_{i j}-\check{\nabla}_{j}|d u|_{\check{g}}^{2}+4 u_{s} u_{k} N^{k s}{ }_{j} .
\end{aligned}
$$

Take $s=\frac{1}{2}$ in (7.31) and (7.32), we get

$$
\begin{aligned}
\check{R}_{i j}= & -\left(\check{\nabla}^{k}-\frac{3}{4} u^{k}\right)\left(N_{i k j}+N_{j k i}\right)-\frac{1}{2}\left(\check{\nabla}^{2} u\right)_{i j}+\frac{1}{2}\left(\check{\nabla}^{2} u\right)_{J i, J j}-\frac{1}{4} \check{\Delta} u \cdot \check{g}_{i j} \\
& +\frac{1}{4} u_{J i} u_{J j}+2\left(N_{-}^{2}\right)_{i j}-2\left(N_{+}^{2}\right)_{i j}, \\
\check{R}= & -\frac{3}{2} \check{\Delta} u+\frac{1}{4}|d u|_{\check{g}}^{2}-|N|_{\breve{g}}^{2}, \\
\check{\nabla}^{k} N_{k i j}= & \frac{1}{4} u^{k} N_{k i j} .
\end{aligned}
$$

Using (7.38) and (7.40), Equation (7.37) can be rearranged as

$$
\begin{aligned}
L_{0}(S)_{j}= & 3 u_{j} \check{\Delta} u+2 u^{i}\left(\left(\check{\nabla}^{2} u\right)_{J i, J j}-\left(\check{\nabla}^{2} u\right)_{i j}\right)-4 u^{s} \check{\nabla}^{i}\left(N_{j i s}+N_{s i j}+3 N_{i j s}\right) \\
& +8\left(\check{\nabla}^{2} u\right)_{s k} N^{k s}{ }_{j}-8 \check{\nabla}^{i}\left(N_{-}^{2}\right)_{i j}+4 u_{s} u_{k} N^{k s}{ }_{j} \\
= & 4 u^{s} \check{R}_{s j}+4 u_{j} \check{\Delta} u+8\left(\check{\nabla}^{2} u\right)_{s k} N^{k s}{ }_{j}-8 \check{\nabla}^{i}\left(N_{-}^{2}\right)_{i j}+4 u_{s} u_{k} N^{k s}{ }_{j} \\
& +8 u^{s}\left(\left(N_{+}^{2}\right)_{s j}-\left(N_{-}^{2}\right)_{s j}\right) \\
= & 4 u^{s} \check{R}_{s j}-\frac{8}{3} u_{j} \check{R}+8\left(\check{\nabla}^{2} u\right)_{s k} N^{k s}{ }_{j}-8 \check{\nabla}^{i}\left(N_{-}^{2}\right)_{i j}+4 u_{s} u_{k} N^{k s}{ }_{j} \\
& +8 u^{s}\left(\left(N_{+}^{2}\right)_{s j}-\left(N_{-}^{2}\right)_{s j}\right)+\frac{2}{3} u_{j}\left(|d u|_{\breve{g}}^{2}-4|N|_{\breve{g}}^{2}\right) .
\end{aligned}
$$


By (17.38), we also know that

$$
\begin{aligned}
\left(\check{\nabla}^{2} u_{s k}\right) N_{j}^{k s}= & -\left(\check{\nabla}^{p}-\frac{3}{4} u^{p}\right)\left(N_{s p k}+N_{k p s}\right) N_{j}^{k s}-\check{R}_{s k} N_{j}^{k s}-\frac{1}{4} u^{k} u^{s} N_{k s j} \\
= & -\check{R}_{s k} N^{k s}{ }_{j}-\check{\nabla}^{p}\left(2 N_{k p s}+N_{p s k}\right) N_{j}^{k s}-\frac{3}{4} u^{p}\left(N_{+}^{2}+N_{-}^{2}\right)_{p j}-\frac{1}{4} u^{k} u^{s} N_{k s j} \\
= & -\check{R}_{s k} N^{k s}{ }_{j}+2 N_{j}^{k s} \check{\nabla}^{p} N_{k s p}-\frac{1}{2} u^{p}\left(N_{-}^{2}+2 N_{+}^{2}\right)_{p j}-\frac{1}{4} u^{k} u^{s} N_{k s j} \\
= & -\check{R}_{s k} N^{k s}{ }_{j}+2 \check{\nabla}^{p}\left(N_{+}^{2}\right)_{p j}-2 N^{k s p} \check{\nabla}_{p} N_{k s j}-\frac{1}{2} u^{p}\left(N_{-}^{2}+2 N_{+}^{2}\right)_{p j}-\frac{1}{4} u^{k} u^{s} N_{k s j} \\
= & -\check{R}_{s k} N^{k s}{ }_{j}+\check{\nabla}^{i}\left(N_{-}^{2}\right)_{i j}-2 N^{k s p} \check{\nabla}_{p} N_{k s j}+\frac{1}{4} \check{\nabla}_{j}|N|_{\check{g}}^{2}-\frac{1}{4} u^{k} u^{s} N_{k s j} \\
& -\frac{1}{2} u^{s}\left(N_{-}^{2}+2 N_{+}^{2}\right)_{s j} .
\end{aligned}
$$

Plugging (7.42) in (7.41), we get

$$
\begin{aligned}
L_{0}(S)_{j}= & 4 u^{s} \check{R}_{s j}-8 \check{R}_{s k} N^{k s}{ }_{j}-\frac{8}{3} u_{j} \check{R}-16 N^{k s p} \check{\nabla}_{p} N_{k s j}+2 \check{\nabla}_{j}|N|_{\check{g}}^{2} \\
& -12 u^{s}\left(N_{-}^{2}\right)_{s j}+2 u_{s} u_{k} N^{k s}{ }_{j}+\frac{2}{3} u_{j}\left(|d u|_{\check{g}}^{2}-4|N|_{\check{g}}^{2}\right) .
\end{aligned}
$$

To deal with the remaining second order terms in (7.43), we rewrite (6.64) using $\check{\nabla}$ derivatives as

$$
8 N^{k s p} \check{\nabla}_{p} N_{k s j}+2 u_{j}|N|_{\check{g}}^{2}-2 u^{i}\left(N_{-}^{2}\right)_{i j}=\frac{3}{2} u_{j}|N|_{\check{g}}^{2}+\check{\nabla}_{j}|N|_{\check{g}}^{2}
$$

Incorporating this identity, we see (7.43) becomes

$$
\begin{aligned}
L_{0}(S)_{j}= & 4 u^{s} \check{R}_{s j}-8 \check{R}_{s k} N^{k s}{ }_{j}-\frac{8}{3} u_{j} \check{R}-16 u^{s}\left(N_{-}^{2}\right)_{s j}+2 u_{s} u_{k} N^{k s}{ }_{j} \\
& +\frac{1}{3} u_{j}\left(2|d u|_{\check{g}}^{2}-5|N|_{\check{g}}^{2}\right) .
\end{aligned}
$$

Q.E.D.

Let $Z$ be the zeroth order linear operator defined by

$$
Z(P)_{j}:=2 u^{i} P_{i j}-4 N^{s t}{ }_{j} P_{s t}-\frac{4}{3} u_{j} \check{g}^{s t} P_{s t},
$$

then (7.36) says

$$
L_{0}(S)_{j}=Z\left(2 \check{R}_{* *}\right)_{j}-16 u^{s}\left(N_{-}^{2}\right)_{s j}+2 u_{s} u_{k} N_{j}^{k s}+\frac{1}{3} u_{j}\left(2|d u|_{\check{g}}^{2}-5|N|_{\check{g}}^{2}\right) .
$$


Consider the first linear operator $L_{1}=L_{0}+Z$, then

$$
\begin{aligned}
L_{1}\left(-2 \check{R}_{* *}+S\right)_{j}= & L_{0}\left(-2 \check{R}_{* *}+S\right)_{j}+Z\left(-2 \check{R}_{* *}+S\right)_{j} \\
= & L_{0}(S)_{j}-Z\left(2 \check{R}_{* *}\right)_{j}+Z(S)_{j} \\
= & -16 u^{s}\left(N_{-}^{2}\right)_{s j}+2 u_{s} u_{k} N_{j}^{k s}+\frac{1}{3} u_{j}\left(2|d u|_{\check{g}}^{2}-5|N|_{\check{g}}^{2}\right) \\
& +8 u^{s}\left(2 N_{+}^{2}+N_{-}^{2}\right)_{s j}-2 u_{s} u_{k} N_{j}^{k s}-\frac{2}{3} u_{j}\left(|d u|_{\check{g}}^{2}+2|N|_{\check{g}}^{2}\right) \\
= & -u_{j}|N|_{\check{g}}^{2}
\end{aligned}
$$

is of first order in $\check{g}$. Therefore if we define the first order linear operator $L$ by

$$
L(P)=L_{1}\left(e^{-\frac{3}{2} u} P\right)
$$

then $L$ is an integrability condition for the flow (7.33). Theorem 5 is proved. Q.E.D.

\subsection{Return to the proof of Theorem 2: uniqueness}

It is now easy to establish the uniqueness part in Theorem 2.

Assume that we have two closed, primitive, and positive solutions $\varphi(t)$ and $\varphi^{\prime}(t)$ of the Type IIA flow on some time interval $[0, T)$ for some $T>0$, with the same initial data $\varphi(0)=\varphi^{\prime}(0)$. By Theorem 4 , the corresponding pairs $\left(\varphi(t), \tilde{g}_{\varphi}(t)\right)$ and $\left(\varphi^{\prime}(t), \tilde{g}_{\varphi^{\prime}}(t)\right)$ satisfy the flows in Theorem 4 . Since the geometries $\left(\omega, J_{\varphi}, g_{\varphi}\right)$ and $\left(\omega, J_{\varphi^{\prime}}, g_{\varphi^{\prime}}\right)$ are by definition Type IIA geometries, the corresponding flows for $\check{g}_{\varphi}(t)$ and $\check{g}_{\varphi^{\prime}}(t)$ satisfy the integrability condition in Theorem 5. Since the principal symbols in the flow of $\check{g}_{i j}$ and the integrability condition $L$ are the same up to a multiplicative factor as their counterparts in the Ricci flow, it follows that the flow of $\check{g}_{i j}$ together with the integrability condition $L$ satisfy all the conditions in the Hamilton-Nash-Moser theorem ([29], Theorem 5.1). By the uniqueness part in this theorem, we conclude that $\check{g}_{i j}(t)$ and $\check{g}_{i j}^{\prime}(t)$ must be equal. But then $\varphi$ and $\varphi^{\prime}(t)$ satisfy the same ODE with the same initial data and hence must be equal. Q.E.D.

\subsection{Monotonicity formulas}

Recall that the function $u$ evolves by

$$
\partial_{t} u=e^{u}\left(\Delta u+2|\nabla u|^{2}+|N|^{2}\right) .
$$

From (7.44) one can derive a number of things.

\section{Proposition 3}

$$
\min _{M} u(t) \geq \min _{M} u(0),
$$


Proof of the Proposition: Apply maximum principle to (7.44). Q.E.D.

This proposition can be interpreted that if $\varphi_{0}$ is initially positive, then $\varphi$ stays positive as long as the flow exists. This is because that the only possibility for $\varphi$ leaving the positive cone is that it first hits the wall of degeneracy defined $|\varphi|=0$, which contradicts the above $C^{0}$-estimate.

Like its Type IIB counterpart [20], one has the following monotonicity formulas for the dilaton functional along the flow.

\section{Proposition 4}

$$
\partial_{t} \int_{M} e^{p u} \frac{\omega^{3}}{3 !}=p \int_{M} e^{(p+1) u}\left((1-p)|\nabla u|^{2}+|N|^{2}\right) \frac{\omega^{3}}{3 !} .
$$

If we denote $\int e^{p u}$ by $E_{p}$, then it follows that $E_{p}$ is monotonely non-increasing along the flow for $p<0$ and it is monotonely non-decreasing along the flow for $0<p \leq 1$. In particular, the Hitchin's functional $E_{1}[30]$ is monotonely non-decreasing along the sourcefree Type IIA flow.

\section{Estimates for the Type IIA flow}

Recall that the Type IIA flow becomes the following flow for the pair $(g(t), u(t))$ :

$$
\begin{aligned}
\partial_{t} g_{i j} & =e^{u}\left[-2 R_{i j}+2 \nabla_{i} \nabla_{j} u-4\left(N_{-}^{2}\right)_{i j}+u_{i} u_{j}-u_{J i} u_{J j}+4 u_{p}\left(N_{i}{ }_{j}{ }_{j}+N_{j}{ }^{p} i\right)\right] \\
\left(\partial_{t}-e^{u} \Delta\right) u & =e^{u}\left[2|\nabla u|^{2}+|N|^{2}\right]
\end{aligned}
$$

In this section, we show that if $|u|+|R m(g)| \leq C$ remains bounded on $[0, T)$, then the flow can be extended to $[0, T+\epsilon)$ for some $\epsilon>0$.

To start, we examine some consequences of the boundedness of the Riemann curvature tensor. We note that by equation (6.22), a bound on $R m$ implies a bound on $|N|^{2}$. Therefore we may assume

$$
|u|+|N|^{2}+|R m| \leq C .
$$

Next, since the Ricci curvature Ric is also bounded, so are its $J$-invariant and $J$-antiinvariant parts. From (6.56), we know that the $J$-invariant part of the Ricci curvature $\left(R^{J}\right)_{i j}$ is given by $\left(\operatorname{Ric}^{J}\right)=\left(\nabla^{2} u\right)^{J}-2 N_{-}^{2}$. As $|N|^{2}$ is already bounded, we conclude

that $\frac{1}{2}\left(\nabla^{2} u\right)_{i j}+\frac{1}{2}\left(\nabla^{2} u\right)_{J i, J j}$ is bounded, namely, the $J$-invariant part of $\nabla^{2} u$ is bounded. Consequently $\Delta u$ is also bounded.

Our goal will be to obtain bounds on all derivatives of $u, N, R m$. For this, we must first compute the evolution equations of $\nabla^{k} u, \nabla^{k} N$ and $\nabla^{k} R m$. 


\subsection{The evolution of the derivatives of $u$}

In this section, we compute the evolution of $|\nabla u|^{2}$ and $|\nabla \nabla u|^{2}$.

\subsubsection{The evolution of the gradient of $u$}

We start with

$$
\partial_{t}|\nabla u|^{2}=2 g^{i j} \nabla_{i} \dot{u} \nabla_{j} u-g^{i a} \dot{g}_{a b} g^{b j} u_{i} u_{j}
$$

The differentiated evolution of $u$ is

$$
\nabla_{i} \dot{u}=e^{u}\left(\nabla_{i} \Delta u+2 \nabla_{i}|\nabla u|^{2}+\nabla_{i}|N|^{2}\right)+e^{u}\left(\Delta u+2|\nabla u|^{2}+|N|^{2}\right) u_{i}
$$

Commuting derivatives

$$
\nabla_{i} \Delta u=g^{p q} \nabla_{i} \nabla_{p} \nabla_{q} u=\Delta \nabla_{i} u-g^{p q} R_{i p}{ }^{\lambda}{ }^{\lambda} u_{\lambda}
$$

Therefore the first term in (8.3)

$$
\begin{aligned}
2 g^{i j} \nabla_{i} \dot{u} \nabla_{j} u= & 2 e^{u}\left(g^{i j} \Delta \nabla_{i} u \nabla_{j} u-g^{p q} R_{p}{ }^{i \lambda}{ }_{q} u_{\lambda} u_{i}+2 g^{i j} \nabla_{i}|\nabla u|^{2} u_{j}+g^{i j} \nabla_{i}|N|^{2} u_{j}\right) \\
& +2 e^{u}\left(\Delta u+2|\nabla u|^{2}+|N|^{2}\right)|\nabla u|^{2} \\
= & e^{u}\left(\Delta|\nabla u|^{2}-2|\nabla \nabla u|^{2}-2 R^{i \lambda} u_{\lambda} u_{i}+8(\nabla \nabla u)^{i j} u_{i} u_{j}+2 g^{i j} \nabla_{i}|N|^{2} u_{j}\right. \\
& \left.+2 \Delta u|\nabla u|^{2}+4|\nabla u|^{4}+2|N|^{2}|\nabla u|^{2}\right)
\end{aligned}
$$

The second term in (8.3) is

$$
\begin{aligned}
& -g^{i a} \dot{g}_{a b} g^{b j} u_{i} u_{j}=e^{u}\left[2 R^{i j}-2 \nabla^{i} \nabla^{j} u+4\left(N_{-}^{2}\right)^{i j}-4 u_{p}\left(N^{i p j}+N^{j p i}\right)\right] u_{i} u_{j} \\
& -e^{u}|\nabla u|^{4}+e^{u}\left[\omega^{a i} u_{a} u_{i}\right]\left[\omega^{b j} u_{b} u_{j}\right] \\
& =e^{u}\left[2 R^{i j}-2(\nabla \nabla u)^{i j}+4\left(N_{-}^{2}\right)^{i j}\right] u_{i} u_{j}-e^{u}|\nabla u|^{4}
\end{aligned}
$$

The term $\left(N^{i p j}+N^{j p i}\right) u_{i} u_{j}$ vanishes by $N^{i p j}=-N^{j i p}-N^{p j i}$ and $N^{j i p}=-N^{p j i}+N^{i j p}$. Altogether, (8.3) becomes

$$
\begin{aligned}
\left(\partial_{t}-e^{u} \Delta\right)|\nabla u|^{2}= & e^{u}\left[-2|\nabla \nabla u|^{2}+2 g^{i j} \nabla_{i}|N|^{2} u_{j}+2 \Delta u|\nabla u|^{2}+3|\nabla u|^{4}\right. \\
& \left.+2|N|^{2}|\nabla u|^{2}+6\left(\nabla^{2} u\right)^{i j} u_{i} u_{j}+4\left(N_{-}^{2}\right)^{i j} u_{i} u_{j}\right]
\end{aligned}
$$

The identity $N_{-}^{2}=2 N_{+}^{2}-\frac{1}{4}|N|^{2} g$ implies

$$
\begin{aligned}
\left(\partial_{t}-e^{u} \Delta\right)|\nabla u|^{2}= & e^{u}\left[-2|\nabla \nabla u|^{2}+2 g^{i j} \nabla_{i}|N|^{2} u_{j}+2 \Delta u|\nabla u|^{2}+3|\nabla u|^{4}\right. \\
& \left.+|N|^{2}|\nabla u|^{2}+6\left(\nabla^{2} u\right)^{i j} u_{i} u_{j}+8\left(N_{+}^{2}\right)^{i j} u_{i} u_{j}\right]
\end{aligned}
$$




\subsubsection{The evolution of the Hessian of $u$}

We use as usual the notation $u_{i j}=\nabla_{i} \nabla_{j} u=\left(\nabla^{2} u\right)_{i j}$. The variation of the Hessian is

$$
\partial_{t} u_{i p}=\nabla_{i} \nabla_{p} \dot{u}-\dot{\Gamma}_{i p}^{\lambda} u_{\lambda}
$$

Differentiating (8.4)

$$
\begin{aligned}
\nabla_{p} \nabla_{i} \dot{u}= & e^{u}\left[\nabla_{p} \nabla_{i} \Delta u+2 \nabla_{p} \nabla_{i}|\nabla u|^{2}+\nabla_{p} \nabla_{i}|N|^{2}\right]+e^{u}\left[\Delta u+2|\nabla u|^{2}+|N|^{2}\right] u_{i p} \\
& +e^{u}\left[\nabla_{i} \Delta u+2 \nabla_{i}|\nabla u|^{2}+\nabla_{i}|N|^{2}\right] u_{p}+(i \leftrightarrow p)
\end{aligned}
$$

Commuting derivatives, we see that

$$
\begin{aligned}
\nabla_{p} \nabla_{i} \Delta u & =g^{a b} \nabla_{p} \nabla_{a} \nabla_{i} \nabla_{b} u-g^{a b} \nabla_{p}\left(R_{i a}{ }^{\lambda}{ }_{b} u_{\lambda}\right) \\
& =g^{a b} \nabla_{a} \nabla_{p} \nabla_{i} \nabla_{b} u-g^{a b} R_{a p}{ }^{\lambda}{ }_{i} u_{\lambda b}-g^{a b} R_{a p}{ }^{\lambda}{ }_{b} u_{i \lambda}-g^{a b} \nabla_{p}\left(R_{i a}{ }^{\lambda}{ }_{b} u_{\lambda}\right) \\
& =\Delta \nabla_{p} \nabla_{i} u-g^{a b} \nabla_{a}\left(R_{p b}{ }^{\lambda}{ }_{i} u_{\lambda}\right)-g^{a b} R_{a p}{ }^{\lambda}{ }_{i} u_{\lambda b}-g^{a b} R_{a p}{ }^{\lambda}{ }_{b} u_{i \lambda}-g^{a b} \nabla_{p}\left(R_{i a}{ }_{b}{ }_{b} u_{\lambda}\right)
\end{aligned}
$$

Therefore

$$
\begin{aligned}
\nabla_{p} \nabla_{i} \dot{u}= & e^{u} \Delta u_{i p}+e^{u}\left[4 g^{a b} u_{i a} u_{p b}\right] \\
& +e^{u}\left[\nabla R m * \nabla u+\nabla^{2} N * N+\nabla^{3} u * \nabla u\right] \\
& +e^{u}\left[\nabla^{2} u * \mathcal{O}(\nabla u, R m, N)+\nabla N * \nabla N+\nabla N * N * \nabla u\right]
\end{aligned}
$$

Here we used the identity $R=\Delta u+|N|^{2}$ on the term $e^{u} \Delta u u_{i p}$.

Before further proceeding the computation, we explain the notations used in the above formula. The terms written as $\alpha * \beta$ represent contractions of the tensors $\alpha$ and $\beta$ which are linear in both $\alpha, \beta$. In later computation, we will also use $(\alpha+\gamma) *(\beta+\eta)$ to represent the linear contractions among the tensors $\alpha, \beta, \gamma$ and $\eta$. The notation $\mathcal{O}(\nabla u, R m, N)$ indicates terms which only depend on $\nabla u, R m$ and $N$ (but the dependence may be nonlinear). We will soon prove a gradient estimate $|\nabla u| \leq C$, so that $\mathcal{O}(\nabla u, R m, N)$ will be treated as bounded terms.

Next,

$$
-\dot{\Gamma}_{i p}^{\lambda} u_{\lambda}=-\frac{g^{\lambda \mu}}{2}\left(-\nabla_{\mu} \dot{g}_{i p}+\nabla_{p} \dot{g}_{\mu i}+\nabla_{i} \dot{g}_{\mu p}\right) u_{\lambda}
$$

Since

$$
\nabla_{\mu} \dot{g}_{i j}=\nabla_{\mu}\left[e^{u}\left(-2 R_{i j}+2 \nabla_{i} \nabla_{j} u-4\left(N_{-}^{2}\right)_{i j}+u_{i} u_{j}-u_{J i} u_{J j}+4 u_{p}\left(N_{i}^{p}{ }_{j}+N_{j}^{p}{ }_{i}\right)\right)\right]
$$


we get

$$
\begin{aligned}
-\dot{\Gamma}_{i p}^{\lambda} u_{\lambda}= & e^{u}\left[\nabla R m * \nabla u+\nabla^{3} u * \nabla u\right]+\mathcal{O}(\nabla u, R m, N) \\
& +e^{u}\left[\nabla^{2} u * \mathcal{O}(\nabla u, R m, N)+\nabla N *(N+\nabla u) * \nabla u\right]
\end{aligned}
$$

Therefore

$$
\begin{aligned}
\left(\partial_{t}-e^{u} \Delta\right) u_{i p}= & e^{u}\left[4 g^{a b} u_{i a} u_{p b}\right]+\mathcal{O}(\nabla u, R m, N) \\
& +e^{u}\left[\nabla R m * \nabla u+\nabla^{2} N * N+\nabla^{3} u * \nabla u\right] \\
& +e^{u}\left[\nabla^{2} u * \mathcal{O}(\nabla u, R m, N)+\nabla N * \nabla N+\nabla N *(N+\nabla u) * \nabla u\right]
\end{aligned}
$$

Next, we compute

$$
\begin{aligned}
\left(\partial_{t}-e^{u} \Delta\right)\left|\nabla^{2} u\right|^{2}= & 2 g^{i j} g^{p q}\left(\partial_{t}-e^{u} \Delta\right) u_{i p} u_{j q}-2 e^{u}\left|\nabla^{3} u\right|^{2} \\
& -g^{i a} \dot{g}_{a b} g^{b j} g^{p q} u_{i p} u_{j q}-g^{i j} g^{p a} \dot{g}_{a b} g^{b q} u_{i p} u_{j q}
\end{aligned}
$$

The first term is then

$$
\begin{aligned}
2 g^{i j} g^{p q}\left(\partial_{t}-e^{u} \Delta\right) u_{i p} u_{j q}= & e^{u}\left[8 g^{a b} u_{i a} u_{p b}\left(\nabla^{2} u\right)^{i p}\right]+\mathcal{O}(\nabla u, R m, N) * \nabla^{2} u \\
& +e^{u}\left[\nabla R m * \nabla u+\nabla^{2} N * N+\nabla^{3} u * \nabla u\right] * \nabla^{2} u \\
& +e^{u}\left[\nabla^{2} u * \mathcal{O}(\nabla u, R m, N)+\nabla N * \nabla N+\nabla N *(N+\nabla u) * \nabla u\right] * \nabla^{2} u
\end{aligned}
$$

Since $\dot{g}_{a b}=2 e^{u} u_{a b}+\mathcal{O}(\nabla u, R m, N)$

$$
\begin{aligned}
\left(\partial_{t}-e^{u} \Delta\right)\left|\nabla^{2} u\right|^{2}= & e^{u}\left[4 g^{a b} u_{i a} u_{p b}\left(\nabla^{2} u\right)^{i p}\right]-2 e^{u}\left|\nabla^{3} u\right|^{2}+\mathcal{O}(\nabla u, R m, N) * \nabla^{2} u \\
& +e^{u}\left[\nabla R m * \nabla u+\nabla^{2} N * N+\nabla^{3} u * \nabla u\right] * \nabla^{2} u \\
& +e^{u}\left[\nabla^{2} u * \mathcal{O}(\nabla u, R m, N)+\nabla N * \nabla N+\nabla N *(N+\nabla u) * \nabla u\right] * \nabla^{2} u
\end{aligned}
$$

\subsection{The evolution of the Nijenhuis tensor: proof of Theorem 6)(a)}

\subsubsection{Rewriting the flow of the complex structure}

The almost complex structure is given by $J^{k}{ }_{j}=\omega^{k i} g_{i j}$. Therefore, $\partial_{t} J^{k}{ }_{j}=\omega^{k i} \partial_{t} g_{i j}$. By substituting equation (6.53) for the Ricci curvature $R_{i j}$ into the flow of metric $\partial_{t} g_{i j}$ (8.1), 
we obtain

$$
\begin{aligned}
\partial_{t} J_{j}^{k}= & e^{u} \omega^{k i}\left\{4 \mathfrak{D}_{p} N_{i}{ }^{p}{ }_{j}-J^{p}{ }_{i} J^{q}{ }_{j} \nabla_{q} \nabla_{p} u+\nabla_{i} \nabla_{j} u\right. \\
& \left.+u_{i} u_{j}-u_{J i} u_{J j}+2 u_{p} N_{j}{ }^{p}{ }_{i}+6 u_{p} N_{i}{ }^{p}{ }_{j}\right\}
\end{aligned}
$$

This simplifies to

$$
\begin{aligned}
\partial_{t} J_{j}^{k}= & e^{u}\left\{4 J^{k}{ }_{q} \mathfrak{D}_{p} N^{q p}{ }_{j}-J^{q}{ }_{j} \nabla_{q} \nabla^{k} u+J^{k}{ }_{q} \nabla^{q} \nabla_{j} u\right. \\
& \left.+u^{J k} u_{j}-u^{k} u_{J j}+2 u_{p} N_{j}{ }^{p, J k}+6 u_{p} N^{J k, p}{ }_{j}\right\} .
\end{aligned}
$$

Converting covariant derivatives using $\nabla_{\ell} V^{p}=\mathfrak{D}_{\ell} V^{p}+N_{\ell \lambda}^{p} V^{\lambda}$, we obtain

$$
\begin{aligned}
4 J^{k}{ }_{q} \mathfrak{D}_{p} N^{q p}{ }_{j} & =-4 J^{k}{ }_{q} \mathfrak{D}_{p} N^{q}{ }_{j}{ }^{p} \\
& =-4 J^{k}{ }_{q}\left(\nabla_{p} N^{q}{ }_{j}{ }^{p}-N_{p \lambda}{ }^{q} N^{\lambda}{ }_{j}{ }^{p}+N_{p j}{ }^{\lambda} N^{q}{ }_{\lambda}^{p}-N_{p \lambda}{ }^{p} N^{q}{ }_{j}{ }^{\lambda}\right)
\end{aligned}
$$

Using the symmetries of the Nijenhuis tensor, this is

$$
\begin{aligned}
4 J_{q}^{k} \mathfrak{D}_{p} N_{j}^{q p} & =-4 J_{q}^{k} \nabla_{p} N^{q}{ }_{j} p-4 N_{p \lambda}{ }^{J k} N^{\lambda p}{ }_{j}+4 N_{j}^{p \lambda}{ }_{j}{ }^{J k}{ }_{\lambda p} \\
& =-4 J_{q}^{k} \nabla_{p} N^{q}{ }_{j}{ }^{p}-4 N_{p \lambda}{ }^{J k} N^{\lambda p}{ }_{j}+4 N^{p \lambda}{ }_{j}\left(-N_{p}{ }^{J k}{ }_{\lambda}-N_{\lambda p}{ }^{J k}\right) \\
& =-4 J^{k}{ }_{q} \nabla_{p} N^{q}{ }_{j}{ }^{p}-8\left(N_{-}^{2}\right)^{J k}{ }_{j}+4\left(N_{+}^{2}\right)^{J k}{ }_{j}
\end{aligned}
$$

using $\left(N_{+}^{2}\right)_{i j}=N^{p \lambda}{ }_{i} N_{p \lambda j}$ and $\left(N_{-}^{2}\right)_{i j}=N^{p \lambda}{ }_{i} N_{\lambda p j}$. Thus

$$
\begin{aligned}
\partial_{t} J_{j}^{k}= & e^{u}\left\{-4 J_{q}^{k} \nabla_{p} N_{j}^{q}{ }_{j}^{p}-J_{j}^{q} \nabla_{q} \nabla^{k} u+J_{q}^{k} \nabla^{q} \nabla_{j} u\right. \\
& \left.+u^{J k} u_{j}-u^{k} u_{J j}+2 u_{p} N_{j}{ }^{p, J k}+6 u_{p} N^{J k, p}{ }_{j}-8\left(N_{-}^{2}\right)^{J k}+4\left(N_{+}^{2}\right)^{J k}{ }_{j}\right\} .
\end{aligned}
$$

\subsubsection{A first formulation of the evolution of the Nijenhuis tensor}

We start with the identity

$$
\nabla_{i} J_{j}^{k}=-2 N_{i j}{ }^{J k}
$$

which follows from the formula relating $\nabla$ to $\mathfrak{D}$ and $N_{i, J j}{ }^{k}=-N_{i j}{ }^{J k}$. Indeed,

$$
\nabla_{i} J^{k}{ }_{j}=\mathfrak{D}_{i} J^{k}{ }_{j}+N_{i \lambda}{ }^{k} J^{\lambda}{ }_{j}-J^{k}{ }_{\lambda} N_{i j}{ }^{\lambda}=-2 N_{i j}{ }^{J k} .
$$

We can expand (8.20) and obtain

$$
J^{k}{ }_{p} N_{i j}{ }^{p}=-\frac{1}{2} \nabla_{i} J^{k}{ }_{j}=-\frac{1}{2} \partial_{i} J^{k}{ }_{j}-\frac{1}{2}\left(\Gamma_{i \lambda}^{k} J^{\lambda}{ }_{j}-J_{\lambda}^{k} \Gamma_{i j}^{\lambda}\right) .
$$


Differentiating this gives

$$
\dot{J}_{p}^{k} N_{i j}{ }^{p}+J_{p}^{k} \dot{N}_{i j}{ }^{p}=-\frac{1}{2} \nabla_{i} \dot{J}_{j}^{k}-\frac{1}{2}\left(\dot{\Gamma}_{i \lambda}^{k} J_{j}^{\lambda}-J_{\lambda}^{k} \dot{\Gamma}_{i j}^{\lambda}\right),
$$

which leads to

$$
\partial_{t} N_{i j}{ }^{\ell}=\frac{1}{2} J^{\ell}{ }_{k} \nabla_{i} \dot{J}^{k}{ }_{j}+J_{k}^{\ell} \dot{J}^{k}{ }_{p} N_{i j}{ }^{p}+\frac{1}{2}\left(J^{\ell}{ }_{k} \dot{\Gamma}_{i \lambda}^{k} J^{\lambda}{ }_{j}+\dot{\Gamma}_{i j}^{\ell}\right) .
$$

We will introduce some notation to group terms. We first introduce the tensor $Z$ given by

$$
Z_{i j}{ }^{\jmath \ell}=J^{\ell}{ }_{r} \dot{\Gamma}_{i n}^{r} J_{j}^{n}+\dot{\Gamma}^{\ell}{ }_{i j} .
$$

Next, we denote $\dot{j}^{k}{ }_{j}=e^{u} E^{k}{ }_{j}$, where by (8.19),

$$
\begin{aligned}
E^{k}{ }_{j}= & -4 J^{k}{ }_{q} \nabla_{p} N^{q}{ }_{j}{ }^{p}-J^{q}{ }_{j} \nabla_{q} \nabla^{k} u+J^{k}{ }_{q} \nabla^{q} \nabla_{j} u \\
& +J^{k}{ }_{p} u^{p} u_{j}-J^{p}{ }_{j} u^{k} u_{p}+2 J^{k}{ }_{\ell} u_{p} N_{j}{ }^{p \ell}+6 J^{k}{ }_{\ell} u_{p} N^{\ell p}{ }_{j} \\
& -8 J^{k}{ }_{\ell}\left(N_{-}^{2}\right)^{\ell}{ }_{j}+4 J^{k}{ }_{\ell}\left(N_{+}^{2}\right)^{\ell}{ }_{j} .
\end{aligned}
$$

We write

$$
\begin{aligned}
\nabla_{i} E^{k}{ }_{j}= & -4 J^{k}{ }_{q} \nabla_{i} \nabla_{p} N^{q}{ }_{j}{ }^{p}-J^{q}{ }_{j} \nabla_{i} \nabla_{q} \nabla^{k} u+J^{k}{ }_{q} \nabla_{i} \nabla^{q} \nabla_{j} u \\
& +J^{k}{ }_{p} \nabla_{i}\left(u^{p} u_{j}\right)-J^{p}{ }_{j} \nabla_{i}\left(u^{k} u_{p}\right)+Y_{i}^{k}{ }_{j},
\end{aligned}
$$

where

$$
\begin{aligned}
Y_{r}{ }_{j}{ }_{j}= & -4 \nabla_{r} J_{q}^{k} \nabla_{p} N^{q}{ }_{j}{ }^{p}-\nabla_{r} J^{q}{ }_{j} \nabla_{q} \nabla^{k} u+\nabla_{r} J_{q}^{k} \nabla^{q} \nabla_{j} u \\
& +\nabla_{r} J^{k}{ }_{p} u^{p} u_{j}-\nabla_{r} J^{p}{ }_{j} u^{k} u_{p}+2 \nabla_{r}\left(J^{k}{ }_{\ell} u_{p} N_{j}{ }^{p \ell}\right)+6 \nabla_{r}\left(J^{k}{ }_{\ell} u_{p} N^{\ell p}{ }_{j}\right) \\
& -8 \nabla_{r}\left(J^{k}{ }_{\ell}\left(N_{-}^{2}\right)^{\ell}{ }_{j}\right)+4 \nabla_{r}\left(J^{k}{ }_{\ell}\left(N_{+}^{2}\right)^{\ell}{ }_{j}\right)
\end{aligned}
$$

Therefore

$$
\begin{aligned}
J_{k}^{\ell} \nabla_{i} \dot{J}_{j}^{k}= & J_{k}^{\ell} \nabla_{i} e^{u} E^{k}{ }_{j}+J_{k}^{\ell} e^{u} \nabla_{i} E^{k}{ }_{j} \\
= & e^{u}\left[4 \nabla_{i} \nabla_{p} N^{\ell}{ }_{j}{ }^{p}-J^{\ell}{ }_{k} J^{q}{ }_{j} \nabla_{i} \nabla_{q} \nabla^{k} u-\nabla_{i} \nabla^{\ell} \nabla_{j} u\right. \\
& \left.-\nabla_{i}\left(u^{\ell} u_{j}\right)-J^{\ell}{ }_{k} J^{p}{ }_{j} \nabla_{i}\left(u^{k} u_{p}\right)+J^{\ell}{ }_{k} Y_{i}{ }_{j}{ }_{j}+u_{i} E^{J \ell}{ }_{j}\right] .
\end{aligned}
$$

Substituting this,

$$
\begin{aligned}
\partial_{t} N_{i j}{ }^{\ell}= & e^{u}\left[-2 \nabla_{i} \nabla_{p} N^{\ell p}{ }_{j}-\frac{1}{2} J_{k}^{\ell}{ }_{k}{ }_{j} \nabla_{i} \nabla_{q} \nabla^{k} u-\frac{1}{2} \nabla_{i} \nabla^{\ell} \nabla_{j} u\right. \\
& -\frac{1}{2} \nabla_{i}\left(u^{\ell} u_{j}\right)-\frac{1}{2} J^{\ell}{ }_{k} J^{p}{ }_{j} \nabla_{i}\left(u^{k} u_{p}\right)+\frac{1}{2} J^{\ell}{ }_{k} Y_{i}{ }^{k}{ }_{j} \\
& \left.+\frac{1}{2} u_{i} E^{J \ell}{ }_{j}+J^{\ell}{ }_{k} E^{k}{ }_{p} N_{i j}{ }^{p}\right]+\frac{1}{2} Z_{i j}{ }^{J \ell} .
\end{aligned}
$$


To interpret the highest order terms, we will need the following identity. We claim:

$$
\begin{aligned}
\Delta N_{i j \ell}= & -2 \nabla_{i} \nabla_{p} N_{\ell}{ }^{p}{ }_{j}-\nabla_{i} R_{\ell j}-\frac{1}{2} \nabla^{p}\left(R_{p i j \ell}-R_{p, i, J j, J \ell}\right) \\
& +\left[\nabla_{i}, \nabla_{p}\right] N^{p}{ }_{\ell j}+\frac{1}{2} \nabla_{i} \nabla_{\ell} \nabla_{j} u+\frac{1}{2} \nabla_{i}\left(J^{p}{ }_{\ell} J^{q}{ }_{j} \nabla_{p} \nabla_{q} u\right) \\
& +2\left[\nabla^{p}\left(N_{p j}{ }^{r} N_{i \ell r}\right)-\nabla^{p}\left(N_{i j}{ }^{r} N_{p \ell r}\right)+\nabla_{i}\left(N_{-}^{2}\right)_{\ell j}-\nabla_{i}\left(N_{+}^{2}\right)_{\ell j}\right] .
\end{aligned}
$$

We assume identity (8.31) for now and give the proof in 98.2 .3 . The evolution of $N$ becomes

$$
\begin{aligned}
\partial_{t} N_{i j}^{\ell}= & e^{u}\left[\Delta N_{i j}{ }^{\ell}-\nabla_{i} \nabla^{\ell} \nabla_{j} u+\nabla_{i} R_{\ell j}+\frac{1}{2} \nabla^{p}\left(R_{p i j \ell}-R_{p, i, J j, J \ell}\right)\right. \\
& -\frac{1}{2}\left(u_{i} u_{j} u^{\ell}+u_{i} u_{J j} u^{J \ell}\right)+(\mathrm{IIa})_{i j}{ }^{\ell}+\frac{e^{-u}}{2} Z_{i j}{ }^{J \ell} \\
& \left.+R m * N+\nabla N *(N+\nabla u)+N^{3}+N^{2} * \nabla u+N *(\nabla u)^{2}\right]
\end{aligned}
$$

where terms involving $\nabla \nabla u$ will need to be tracked for future use, and are given explicitly by

$$
\begin{aligned}
(\text { IIa })_{i j}{ }^{\ell}= & -\frac{1}{2} \nabla_{i}\left(u^{\ell} u_{j}\right)-\frac{1}{2} J^{\ell}{ }_{k} J^{p}{ }_{j} \nabla_{i}\left(u^{k} u_{p}\right)-\frac{1}{2} g^{r \ell} \nabla_{i}\left(J^{p}{ }_{r} J_{j}{ }_{j}\right)\left(\nabla^{2} u\right)_{p q} \\
& +\frac{1}{2} J^{\ell}{ }_{k}\left[-\nabla_{i} J^{q}{ }_{j}\left(\nabla^{2} u\right)_{q}{ }^{k}+\nabla_{i} J^{k}{ }_{q}\left(\nabla^{2} u\right)^{q}{ }_{j}+2 J^{k}{ }_{r}\left(\nabla^{2} u\right)_{i p} N_{j}{ }^{p r}+6 J^{k}{ }_{r}\left(\nabla^{2} u\right)_{i p} N^{r p}{ }_{j}\right] \\
& +\frac{1}{2} u_{i} J^{\ell}{ }_{k}\left[-J^{q}{ }_{j}\left(\nabla^{2} u\right)^{k}{ }_{q}+J^{k}{ }_{q}\left(\nabla^{2} u\right)^{q}{ }_{j}\right] \\
& +J^{\ell}{ }_{k}\left[-J^{q}{ }_{p}\left(\nabla^{2} u\right)_{q}{ }^{k}+J^{k}{ }_{q}\left(\nabla^{2} u\right)^{q}{ }_{p}\right] N_{i j}{ }^{p}
\end{aligned}
$$

which, using $\nabla_{i} J^{k}{ }_{j}=-2 N_{i j}{ }^{J k}$ and simplifying, become

$$
\begin{aligned}
(\text { IIa })_{i j}{ }^{\ell}= & -\frac{1}{2} \nabla_{i}\left(u^{\ell} u_{j}\right)-\frac{1}{2} J^{\ell}{ }_{k} J^{p}{ }_{j} \nabla_{i}\left(u^{k} u_{p}\right)+N_{i}{ }^{\ell J p}\left(\nabla^{2} u\right)_{p, J j}-N_{i j}{ }^{J q}\left(\nabla^{2} u\right)^{J \ell}{ }_{q} \\
& +N_{i j}{ }^{J q}\left(\nabla^{2} u\right)_{q}{ }^{\ell \ell}+N_{i q}{ }^{\ell}\left(\nabla^{2} u\right)^{q}{ }_{j}-\left(\nabla^{2} u\right)_{i p} N_{j}{ }^{\ell \ell}-3\left(\nabla^{2} u\right)_{i p} N^{\ell p}{ }_{j} \\
& -\frac{1}{2} u_{i}\left[\left(\nabla^{2} u\right)^{J \ell}{ }_{J j}+\left(\nabla^{2} u\right)^{\ell}{ }_{j}\right]-\left[\left(\nabla^{2} u\right)_{J p}{ }^{J \ell}+\left(\nabla^{2} u\right)^{\ell}{ }_{p}\right] N_{i j}{ }^{p} .
\end{aligned}
$$

Next, we claim that

$$
\begin{aligned}
Z_{i j}{ }^{J p}= & \nabla_{i} \dot{g}^{p}{ }_{j}+\frac{1}{2}\left(-\nabla^{p} \dot{g}_{i j}+\nabla_{j} \dot{g}^{p}{ }_{i}\right)+\frac{1}{2}\left(\omega^{r p} J^{n}{ }_{j}-\omega^{n p} J^{r}{ }_{j}\right) \nabla_{r} \dot{g}_{i n} \\
& +\left(N_{i}{ }^{p r} \dot{g}_{j r}+N_{i j} \dot{g}^{p}{ }_{r}\right) .
\end{aligned}
$$


We assume identity (8.35) for now and give the proof later in 88.2 .3 , Substituting the evolution of $g_{i j}$ (8.1) into this expression for $Z_{i j}{ }^{J p}$ and then in our expression for $\partial_{t} N_{i j}^{\ell}$, we obtain

$$
\begin{aligned}
\partial_{t} N_{i j}^{\ell}= & e^{u}\left[\Delta N_{i j}{ }^{\ell}-\nabla_{i} \nabla^{\ell} \nabla_{j} u+\nabla_{i} R^{\ell}{ }_{j}+\frac{1}{2} \nabla^{p}\left(R_{p i j}{ }^{\ell}-g^{\ell r} R_{p, i, J j, J r}\right)\right. \\
& -\nabla_{i} R^{\ell}{ }_{j}-\frac{1}{2}\left(-\nabla^{\ell} R_{i j}+\nabla_{j} R_{i}^{\ell}\right)-\frac{1}{2}\left(\omega^{r \ell} J^{n}{ }_{j}-\omega^{n \ell} J^{r}{ }_{j}\right) \nabla_{r} R_{i n} \\
& +\nabla_{i} \nabla^{\ell} \nabla_{j} u+\frac{1}{2}\left(-\nabla^{\ell} \nabla_{i} \nabla_{j} u+\nabla_{j} \nabla^{\ell} \nabla_{i} u\right)+\frac{1}{2}\left(\omega^{r \ell} J^{n}{ }_{j}-\omega^{n \ell} J^{r}{ }_{j}\right) \nabla_{r} \nabla_{i} \nabla_{n} u \\
& +R i c * \nabla u+(\mathrm{IIa})_{i j}{ }^{\ell}+(\mathrm{IIb})_{i j}{ }^{\ell} \\
& \left.+R m * N+\nabla N *(N+\nabla u)+N^{3}+N^{2} * \nabla u+N *(\nabla u)^{2}\right]
\end{aligned}
$$

where terms of order $(\nabla u)^{3}$ (e.g. $\left.u_{i} u_{j} u^{\ell}\right)$ have cancelled and the additional terms involving $\nabla^{2} u$ are

$$
\begin{aligned}
(\mathrm{IIb})_{i j}{ }^{\ell}= & \frac{1}{2}\left[\nabla_{i}\left(u^{\ell} u_{j}-u^{J \ell} u_{J j}\right)+4\left(\nabla^{2} u\right)_{p i}\left(N^{\ell p}{ }_{j}+N_{j}{ }^{\ell \ell}\right)+2 u_{i}\left(\nabla^{2} u\right)^{\ell}{ }_{j}\right] \\
& -\frac{1}{4}\left[\nabla^{\ell}\left(u_{i} u_{j}-u_{J i} u_{J j}\right)+4\left(\nabla^{2} u\right)_{p}{ }^{\ell}\left(N_{i}{ }^{p}{ }_{j}+N_{j}{ }^{p}{ }_{i}\right)+2 u^{\ell}\left(\nabla^{2} u\right)_{i j}\right] \\
& +\frac{1}{4}\left[\nabla_{j}\left(u_{i} u^{\ell}-u_{J i} u^{J \ell}\right)+4\left(\nabla^{2} u\right)_{p j}\left(N_{i}{ }^{p \ell}+N^{\ell p}{ }_{i}\right)+2 u_{j}\left(\nabla^{2} u\right)_{i}{ }^{\ell}\right] \\
& +\frac{1}{4}\left(\omega^{r \ell} J^{n}{ }_{j}-\omega^{n \ell} J^{r}{ }_{j}\right)\left[\nabla_{r}\left(u_{i} u_{n}-u_{J i} u_{J j}\right)+4\left(\nabla^{2} u\right)_{p r}\left(N_{i}{ }^{p}{ }_{n}+N_{n}{ }^{p}{ }_{i}\right)+2 u_{r}\left(\nabla^{2} u\right)_{i n}\right] \\
& +\left(N_{i}{ }^{\ell r}\left(\nabla^{2} u\right)_{j r}+N_{i j}{ }^{r}\left(\nabla^{2} u\right)^{\ell}{ }_{r}\right) .
\end{aligned}
$$

Since we can commute $\nabla_{\ell} \nabla_{j} \nabla_{i} u=\nabla_{j} \nabla_{\ell} \nabla_{i} u-R_{\ell j}{ }^{p} u_{p}$, the terms of order $\nabla^{3} u$ in (8.36) cancel. We are left with

$$
\begin{aligned}
& \partial_{t} N_{i j}^{\ell}=e^{u}\left[\Delta N_{i j}^{\ell}+\frac{1}{2} \nabla^{p} R_{p i j}^{\ell}-\frac{1}{2} \omega^{n \ell} J_{j}^{r} \nabla^{p} R_{p i r n}\right. \\
& -\frac{1}{2}\left(-\nabla^{\ell} R_{i j}+\nabla_{j} R_{i}^{\ell}\right)-\frac{1}{2}\left(\omega^{r \ell} J_{j}^{n}-\omega^{n \ell} J_{j}^{r}\right) \nabla_{r} R_{i n} \\
& +\frac{1}{2}\left(-R_{j}{ }^{\ell p}{ }_{i} u_{p}+R^{J \ell}{ }_{i}{ }_{J j} u_{p}-R_{J j, i}{ }^{p, J \ell} u_{p}\right)+R i c * \nabla u+(\mathrm{IIa})_{i j}{ }^{\ell}+(\mathrm{IIb})_{i j}{ }^{\ell} \\
& \left.+R m * N+\nabla N *(N+\nabla u)+N^{3}+N^{2} * \nabla u+N *(\nabla u)^{2}\right] .
\end{aligned}
$$

The terms of order $\nabla R m$ also cancel. Indeed, the Bianchi identity is

$$
\nabla^{p} R_{j p i}^{\ell}+\nabla^{\ell} R_{j p i}^{p}+\nabla_{j} R_{p i}^{\ell p}=0
$$

and hence

$$
\frac{1}{2} \nabla^{p} R_{p i j}^{\ell}=\frac{1}{2} \nabla^{p} R_{j}^{\ell}{ }_{p i}=\frac{1}{2}\left(-\nabla^{\ell} R_{i j}+\nabla_{j} R_{i}^{\ell}\right) .
$$


For the terms involving $\omega, J$, the same argument gives

$$
-\frac{1}{2} \omega^{n \ell} J^{r}{ }_{j} \nabla^{p} R_{\text {pirn }}=-\frac{1}{2} \omega^{n \ell} J^{r}{ }_{j} \nabla^{p} R_{\text {nrip }}=\frac{1}{2} \omega^{n \ell} J^{r}{ }_{j}\left(\nabla_{n} R_{r i}-\nabla_{r} R_{\text {in }}\right)
$$

This is the same thing as

$$
-\frac{1}{2} \omega^{n \ell} J_{j}^{r} \nabla^{p} R_{\text {pirn }}=\frac{1}{2}\left(\omega^{r \ell}{J^{n}}_{j}-\omega^{n \ell} J^{r}{ }_{j}\right) \nabla_{r} R_{i n}
$$

Putting these identities back to (8.38), we get the cancellation of the $\nabla R m$ terms. Thus

$$
\begin{aligned}
\partial_{t} N_{i j}{ }^{k}= & e^{u}\left[\Delta N_{i j}{ }^{k}+(R m * \nabla u)_{i j}{ }^{k}+(\mathrm{IIa})_{i j}{ }^{k}+(\mathrm{IIb})_{i j}{ }^{k}\right. \\
& \left.+R m * N+\nabla N *(N+\nabla u)+N^{3}+N^{2} * \nabla u+N *(\nabla u)^{2}\right],
\end{aligned}
$$

where the (II) terms involve $\nabla^{2} u$ and are explicitly given in (8.34) and (8.37) and $R m * \nabla u$ is of the form

$$
(R m * \nabla u)_{i j}{ }^{k}=\frac{1}{2}\left(-R_{j}{ }^{k p}{ }_{i} u_{p}+R^{J k}{ }_{i}^{p}{ }_{J j} u_{p}-R_{J j, i}{ }^{p, J k} u_{p}\right)+(\operatorname{Ric} * \nabla u)_{i j}{ }^{k} .
$$

\subsubsection{Proof of Identity (8.31) and Identity (8.35)}

Proof of identity (8.31): The starting point is the identity (6.11) for the action of $J$ on the Riemann curvature tensor. Recall that in the case $d \omega=0$, (6.4) and (6.5) specialize to $A=N=\mathfrak{T}$, and so the identity (6.11) becomes

$$
\begin{aligned}
R_{i, j, J k, J l}-R_{i j k l} & =2\left(\mathfrak{D}_{i} N_{j k l}-\mathfrak{D}_{j} N_{i k l}+N^{r}{ }_{i j} N_{r k l}\right) \\
& =2\left(\mathfrak{D}_{i} N_{j k l}-\mathfrak{D}_{j} N_{i k l}+N_{j i}{ }^{r} N_{r k l}-N_{i j}{ }^{r} N_{r k l}\right),
\end{aligned}
$$

using $N_{i j k}+N_{k i j}+N_{j k i}=0$. We can convert $\mathfrak{D} N$ to $\nabla N$. For example,

$$
\mathfrak{D}_{i} N_{j k l}=\nabla_{i} N_{j k l}+N_{i j}^{r} N_{r k l}+N_{i k}^{r} N_{j r l}+N_{i l}^{r} N_{j k r} .
$$

After converting $\mathfrak{D}$ to $\nabla$, (8.44) becomes

$$
\nabla_{j} N_{i \ell k}=\nabla_{i} N_{j \ell k}+\frac{1}{2}\left(R_{j i k \ell}-R_{j, i, J k, J \ell}\right)-2 N_{j k}^{r} N_{i \ell r}+2 N_{i k}^{r} N_{j \ell r} .
$$

Differentiating this identity, we obtain

$$
\begin{aligned}
\nabla_{q} \nabla_{p} N_{k i j}= & \nabla_{q} \nabla_{k} N_{p i j}+\frac{1}{2} \nabla_{q}\left(R_{p k j i}-R_{p, k, J j, J i}\right) \\
& -2 \nabla_{q}\left(N_{p j}{ }^{r} N_{k i r}\right)+2 \nabla_{q}\left(N_{k j}{ }^{r} N_{p i r}\right) .
\end{aligned}
$$


By the Bianchi identity,

$$
\begin{aligned}
\Delta N_{i j k}= & -\Delta N_{k i j}+\Delta N_{j i k} \\
= & -\nabla_{p} \nabla_{k} N^{p}{ }_{i j}-\frac{1}{2} \nabla^{p}\left(R_{p k j i}-R_{p, k, J j, J i}\right) \\
& +2 \nabla^{p}\left(N_{p j}{ }^{r} N_{k i r}\right)-2 \nabla^{p}\left(N_{k j}{ }^{r} N_{p i r}\right)+\Delta N_{j i k} .
\end{aligned}
$$

Therefore

$$
\begin{aligned}
-\Delta N_{j i k}+\Delta N_{i j k}= & -\nabla_{k} \nabla_{p} N^{p}{ }_{i j}-\frac{1}{2} \nabla^{p}\left(R_{p k j i}-R_{p, k, J j, J i}\right) \\
& +\left[\nabla_{k}, \nabla_{p}\right] N^{p}{ }_{i j}+2 \nabla^{p}\left(N_{p j}{ }^{r} N_{k i r}\right)-2 \nabla^{p}\left(N_{k j}{ }^{r} N_{p i r}\right) \\
= & \nabla_{k} \nabla_{p} N_{j}{ }^{p}{ }_{i}-\nabla_{k} \nabla_{p} N_{i}{ }^{p}{ }_{j}-\frac{1}{2} \nabla^{p}\left(R_{p k j i}-R_{p, k, J j, J i}\right) \\
& +\left[\nabla_{k}, \nabla_{p}\right] N^{p}{ }_{i j}+2 \nabla^{p}\left(N_{p j}{ }^{r} N_{k i r}\right)-2 \nabla^{p}\left(N_{k j}{ }^{r} N_{p i r}\right)
\end{aligned}
$$

The formula for Ricci curvature in Type IIA geometry given in (6.54) is

$$
\nabla_{p} N_{j}{ }_{i}{ }_{i}=-\nabla_{p} N_{i}{ }^{p}{ }_{j}-R_{i j}+2\left(N_{-}^{2}\right)_{i j}-2\left(N_{+}^{2}\right)_{i j}+\frac{1}{2}\left(\nabla^{2} u\right)_{i j}+\frac{1}{2}\left(\nabla^{2} u\right)_{J i, J j} .
$$

Substituting this into (8.49),

$$
\begin{aligned}
-\Delta N_{j i k}+\Delta N_{i j k}= & -2 \nabla_{k} \nabla_{p} N_{i}{ }^{p}{ }_{j}-\nabla_{k} R_{i j}-\frac{1}{2} \nabla^{p}\left(R_{p k j i}-R_{p, k, J j, J i}\right) \\
& +\left[\nabla_{k}, \nabla_{p}\right] N^{p}{ }_{i j}+\frac{1}{2} \nabla_{k} \nabla_{i} \nabla_{j} u+\frac{1}{2} \nabla_{k}\left(J^{p}{ }_{i} J^{q}{ }_{j} \nabla_{p} \nabla_{q} u\right) \\
& +2 \nabla^{p}\left(N_{p j}{ }^{r} N_{k i r}\right)-2 \nabla^{p}\left(N_{k j}{ }^{r} N_{p i r}\right)+2 \nabla_{k}\left(N_{-}^{2}\right)_{i j}-2 \nabla_{k}\left(N_{+}^{2} \gamma_{\ell j} 51\right)
\end{aligned}
$$

This proves the identity after using $N_{i j k}+N_{k i j}+N_{j k i}=0$. Q.E.D.

Proof of Identity (8.35): The variation of the Christoffel symbol is given by

$$
\dot{\Gamma}_{i n}^{p}=\frac{g^{p s}}{2}\left(-\nabla_{s} \dot{g}_{i n}+\nabla_{i} \dot{g}_{s n}+\nabla_{n} \dot{g}_{i s}\right) .
$$

Thus

$$
\begin{aligned}
Z_{i j}{ }^{p}=\dot{\Gamma}_{i n}^{p} J_{j}^{n}-J_{n}{ }_{n} \dot{\Gamma}^{n}{ }_{i j}= & \frac{g^{p s}}{2}\left(-\nabla_{s} \dot{g}_{i n} J_{j}^{n}+\nabla_{i} \dot{g}_{s n} J_{j}^{n}+J_{j}^{n} \nabla_{n} \dot{g}_{i s}\right) \\
& -\frac{g^{n s}}{2} J^{p}{ }_{n}\left(-\nabla_{s} \dot{g}_{i j}+\nabla_{i} \dot{g}_{s j}+\nabla_{j} \dot{g}_{i s}\right) .
\end{aligned}
$$

Therefore

$$
\begin{aligned}
Z_{i j}{ }^{J \ell}= & \frac{g^{p s}}{2} J^{\ell}{ }_{p}\left(-\nabla_{s} \dot{g}_{i n} J^{n}{ }_{j}+\nabla_{i} \dot{g}_{s n} J^{n}{ }_{j}+J^{n}{ }_{j} \nabla_{n} \dot{g}_{i s}\right) \\
& -\frac{g^{n s}}{2} J^{\ell}{ }_{p} J^{p}{ }_{n}\left(-\nabla_{s} \dot{g}_{i j}+\nabla_{i} \dot{g}_{s j}+\nabla_{j} \dot{g}_{i s}\right),
\end{aligned}
$$


which becomes

$$
\begin{aligned}
Z_{i j}{ }^{J p}= & -\frac{g^{p s}}{2} J^{r}{ }_{s} \nabla_{i} \dot{g}_{r n} J^{n}{ }_{j}+\frac{1}{2}\left(-\nabla^{p} \dot{g}_{i j}+\nabla_{i} \dot{g}^{p}{ }_{j}+\nabla_{j} \dot{g}^{p}{ }_{i}\right) \\
& +\frac{1}{2}\left(\omega^{r p} J^{n}{ }_{j} \nabla_{r} \dot{g}_{i n}-\omega^{n p} J^{r}{ }_{j} \nabla_{r} \dot{g}_{i n}\right) .
\end{aligned}
$$

From the evolution equation of $g$, we have the identity $J_{i}^{r} \dot{g}_{r n} J_{j}^{n}=-\dot{g}_{i j}$. Therefore the first term

$$
-\frac{g^{p s}}{2} J^{r}{ }_{s} \nabla_{i} \dot{g}_{r n} J^{n}{ }_{j}=-\frac{g^{p s}}{2} \nabla_{i}\left(J^{r}{ }_{s} \dot{g}_{r n} J^{n}{ }_{j}\right)+\frac{g^{p s}}{2} \nabla_{i} J_{s}^{r} \dot{g}_{r n} J^{n}{ }_{j}+\frac{g^{p s}}{2} J^{r}{ }_{s} \dot{g}_{r n} \nabla_{i} J^{n}{ }_{j}
$$

simplifies to

$$
-\frac{g^{p s}}{2} J_{s}^{r} \nabla_{i} \dot{g}_{r n} J_{j}^{n}=\frac{1}{2} \nabla_{i} \dot{g}^{p}{ }_{j}+N_{i}{ }^{p r} \dot{g}_{r j}+\dot{g}_{n}^{p} N_{i j}{ }^{n} .
$$

Therefore

$$
\begin{aligned}
Z_{i j}{ }^{J p}= & \nabla_{i} \dot{g}^{p}{ }_{j}+\frac{1}{2}\left(-\nabla^{p} \dot{g}_{i j}+\nabla_{j} \dot{g}^{p}{ }_{i}\right)+\frac{1}{2}\left(\omega^{r p} J^{n}{ }_{j} \nabla_{r} \dot{g}_{i n}-\omega^{n p} J^{r}{ }_{j} \nabla_{r} \dot{g}_{i n}\right) \\
& +N_{i}{ }^{p r} \dot{g}_{r j}+\dot{g}^{p}{ }_{n} N_{i j}{ }^{n} .
\end{aligned}
$$

Q.E.D.

\subsubsection{The evolution of the norm of $N$}

The norm of the Nijenhuis tensor, which is $|N|^{2}=g^{i j} g^{p q} g^{k \ell} N_{i p k} N_{j q \ell}$, evolves by

$$
\begin{aligned}
\left(\partial_{t}-e^{u} \Delta\right)|N|^{2}= & 2 N^{i j}{ }_{k}\left(\partial_{t}-e^{u} \Delta\right) N_{i j}{ }^{k}-2 e^{u}|\nabla N|^{2} \\
& -g^{i r} \dot{g}_{r s} g^{s j} N_{i p k} N_{j}^{p k}-2 g^{k r} \dot{g}_{r s} g^{s \ell} N_{i p k} N^{i p}{ }_{\ell} .
\end{aligned}
$$

By the equation for the evolution of $g_{i j}$ (8.1), this is of the form

$$
\begin{aligned}
\left(\partial_{t}-e^{u} \Delta\right)|N|^{2}= & 2 N^{i j}{ }_{k}\left(\partial_{t}-e^{u} \Delta\right) N_{i j}{ }^{k}-2 e^{u}|\nabla N|^{2} \\
& +e^{u}\left[-2\left(\nabla^{2} u\right)^{i j} N_{i p k} N_{j}{ }^{p k}-4\left(\nabla^{2} u\right)^{k \ell} N_{i p k} N^{i p}{ }_{\ell}\right. \\
& \left.+N^{2} *(R m+N * N+\nabla u * \nabla u+\nabla u * N)\right]
\end{aligned}
$$

Substituting (8.42), we obtain

$$
\begin{aligned}
& \left(\partial_{t}-e^{u} \Delta\right)|N|^{2} \\
= & e^{u}\left[-2|\nabla N|^{2}+2 N^{i j}{ }_{k}(\mathrm{IIa}+\mathrm{IIb})_{i j}{ }^{k}-2\left(\nabla^{2} u\right)^{i j} N_{i p k} N_{j}{ }^{p k}-4\left(\nabla^{2} u\right)^{k \ell} N_{i p k} N^{i p}{ }_{\ell}\right. \\
& +2 N^{i j}{ }_{k}(R m * \nabla u)_{i j}{ }^{k}+R m * N^{2} \\
& \left.+\nabla N * N *(N+\nabla u)+N^{4}+N^{3} * \nabla u+N^{2} *(\nabla u)^{2}\right] .
\end{aligned}
$$


The expressions for the terms (IIa + IIb) are given in (8.34) and (8.37). A calculation can be done to verify that each term $N^{i j}{ }_{k}(\mathrm{IIa})_{i j}{ }^{k}$ and $N^{i j}{ }_{k}(\mathrm{IIb})_{i j}{ }^{k}$ only contributes terms of the type $\left(\nabla^{2} u\right) * N^{2}$ since the others vanish by symmetry. For example, if we denote the terms on each line of (IIb) (8.37) by $(i)+(i i)+(i i i)+(i v)+(v)$, we have

$$
\begin{aligned}
& (i)=\frac{1}{2} N^{i j}{ }_{\ell}\left[\nabla_{i}\left(u^{\ell} u_{j}\right)-J^{\ell}{ }_{r} J^{s}{ }_{j} \nabla_{i}\left(u^{r} u_{s}\right)+4 u_{p i}\left(N^{\ell p}{ }_{j}+N_{j}{ }^{p \ell}\right)+2 u_{i} u^{\ell}{ }_{j}\right] \\
& =-2 u_{p i} N^{i j}{ }_{\ell} N^{\ell}{ }_{j p}-2 u_{p i} N^{i j}{ }_{\ell} N_{j}{ }^{\ell} \\
& =2 u_{i}^{p}\left(N^{\ell i j}+N^{j \ell i}\right) N_{\ell j p}+2 u_{p i}\left(N^{\ell i j}+N^{j \ell i}\right) N_{j \ell p}=0
\end{aligned}
$$

where we used the symmetry $N^{i j}{ }_{\ell}=-N^{i} \ell^{j}$, the identity $N^{i, J j}{ }_{J r}=N^{i j}{ }_{r}$, and the Bianchi identity $N_{i j k}+N_{k i j}+N_{j k i}=0$. The symmetry $N_{\ell}^{i j}=-N_{\ell}^{i}$ allows us to combine the $(i i)+($ iii $)$ terms:

$$
\begin{aligned}
(i i)+(i i i) & =-\frac{1}{2} N^{i j}{ }_{\ell}\left[\nabla^{\ell}\left(u_{i} u_{j}\right)-J_{i}^{r}{ }_{i}{ }_{j} \nabla^{\ell}\left(u_{r} u_{s}\right)+4 u_{p}^{\ell}\left(N_{i}{ }_{j}{ }_{j}+N_{j}{ }_{i}\right)+2 u^{\ell} u_{i j}\right] \\
& =-\frac{1}{2} N^{i j}{ }_{\ell}\left[2 u^{\ell}{ }_{i} u_{j}+4 u_{p}^{\ell}\left(N_{i}{ }_{j}{ }_{j}+N_{j}{ }^{p}\right)+2 u^{\ell} u_{i j}\right] \\
& =2 u_{p}^{\ell}\left(N^{i j}{ }_{\ell} N_{i j}{ }^{p}\right)+2 u_{p}^{\ell}\left(N^{i j}{ }_{\ell} N_{j i}{ }^{p}\right)
\end{aligned}
$$

where we used $N^{J i, J j} \ell=-N_{\ell}^{i j}$. Next,

$$
\begin{aligned}
(i v) & =\frac{1}{2} N^{i j}{ }_{\ell} \omega^{r \ell} J^{n}{ }_{j}\left[\nabla_{r}\left(u_{i} u_{n}\right)-J^{p}{ }_{i} J^{q}{ }_{n} \nabla_{r}\left(u_{p} u_{q}\right)+4 u_{p r}\left(N_{i}{ }_{n}{ }_{n}+N_{n}{ }^{p}{ }_{i}\right)+2 u_{r} u_{i n}\right] \\
& =N^{i j}{ }_{\ell} \omega^{r \ell} J^{n}{ }_{j}\left[u_{r i} u_{n}+u_{i} u_{r n}+2 u_{p r}\left(N_{i}{ }_{n}{ }_{n}+N_{n}{ }^{p}{ }_{i}\right)+u_{r} u_{i n}\right] \\
& =-N^{i, J n}{ }_{J \ell} g^{r \ell}\left[u_{r i} u_{n}+u_{i} u_{r n}+2 u_{p r}\left(N_{i}{ }^{p}{ }_{n}+N_{n}{ }^{p}{ }_{i}\right)+u_{r} u_{i n}\right] \\
& =-N^{i n}{ }_{\ell}\left[u^{\ell}{ }_{i} u_{n}+u_{i} u^{\ell}{ }_{n}+2 u^{\ell}{ }_{p}\left(N_{i}{ }_{n}{ }_{n}+N_{n}{ }_{i}\right)+u^{\ell} u_{i n}\right] \\
& =2 u^{\ell}{ }_{p}\left(N^{i n}{ }_{\ell} N_{i n}{ }^{p}\right)+2 u^{\ell}{ }_{p}\left(N^{i n}{ }_{\ell} N_{n i}{ }^{p}\right) .
\end{aligned}
$$

The computations of the other terms is similar. The result is then

$$
\begin{aligned}
\left(\partial_{t}-e^{u} \Delta\right)|N|^{2}= & e^{u}\left[-2|\nabla N|^{2}+\left(\nabla^{2} u\right) * N^{2}+2 N_{k}^{i j}(R m * \nabla u)_{i j}{ }^{k}\right. \\
& \left.+R m * N^{2}+\nabla N * N *(N+\nabla u)+N^{4}+N^{3} * \nabla u+N^{2} *(\nabla u)^{2}\right]
\end{aligned}
$$

where $\left(\nabla^{2} u\right) \star N^{2}$ is an expression involving $(\nabla u)^{i j}\left(N_{+}^{2}\right)_{i j}$ and $(\nabla u)^{i j}\left(N_{-}^{2}\right)_{i j}$. The expression for the term $(R m * \nabla u)_{i j}{ }^{k}$ is given in (8.43), and using $N^{i, J j}{ }_{J k}=N^{i j}{ }_{k}$ and symmetries of the curvature tensor, it becomes

$$
N^{i j}{ }_{k}(R m * \nabla u)_{i j}{ }^{k}=-R^{p}{ }_{i j k} u_{p} N^{i j k}+N^{i j}{ }_{k}(\operatorname{Ric} * \nabla u)_{i j}{ }^{k} .
$$

By (6.16) , we can convert $R^{p}{ }_{i j k}=\mathfrak{R}^{p}{ }_{i j k}+\nabla N+N^{2}$. Since $\mathfrak{R}^{p}{ }_{i, J j, J k}=\mathfrak{R}^{p}{ }_{i j k}$ and $N^{i, J j, J k}=$ $-N^{i j k}$, the term $\mathfrak{R}_{i j k}^{p} N^{i j k}=0$ by symmetry. Similarly, we can reduce terms of the form 
$N^{i j}{ }_{k} u^{k} R_{i j}=N^{i j}{ }_{k} u^{k} R_{i j}^{-J}$ where $R_{i j}^{-J}$ is the $J$-anti-invariant part of the Ricci tensor given in (6.56) by $\operatorname{Ric}^{-J}=\nabla N+N * N$. Absorbing these terms gives the expression

$$
\begin{aligned}
\left(\partial_{t}-e^{u} \Delta\right)|N|^{2}= & e^{u}\left[-2|\nabla N|^{2}+\left(\nabla^{2} u\right) * N^{2}+R m * N^{2}\right. \\
& \left.+\nabla N * N *(N+\nabla u)+N^{4}+N^{3} * \nabla u+N^{2} *(\nabla u)^{2}\right] .
\end{aligned}
$$

We remark that from this expression, we see that if $|N|^{2}=0$ at the initial time, that $|N|^{2} \equiv 0$ along the flow. To see this, we assume $|N|^{2} \leq 1$ and $|R m|+\left|\nabla^{2} u\right|+|\nabla u| \leq C$ on $[0, \epsilon)$ and apply the maximum principle to $e^{-A t}|N|^{2}$ for $A \gg 1$.

\subsubsection{The evolution of the gradient of $N$}

We compute the evolution of $\nabla N$.

$$
\partial_{t} \nabla_{\ell} N_{i j}{ }^{k}=\nabla_{\ell} \dot{N}_{i j}{ }^{k}-N_{\lambda j}{ }^{k} \dot{\Gamma}_{\ell i}^{\lambda}-N_{i \lambda}{ }^{k} \dot{\Gamma}_{\ell j}^{\lambda}+\dot{\Gamma}_{\ell \lambda}^{k} N_{i j}{ }^{\lambda}
$$

Using the equation (8.1) for $\dot{g}_{i j}$, we can compute the time derivative of the Christoffel symbol as

$$
\begin{aligned}
\dot{\Gamma}_{i j}^{k} & =-\frac{g^{k \mu}}{2}\left(-\nabla_{\mu} \dot{g}_{i j}+\nabla_{j} \dot{g}_{\mu i}+\nabla_{i} \dot{g}_{\mu p}\right) \\
& =e^{u}\left(\nabla R m * g+\nabla^{3} u * g+\left(\nabla N+\nabla^{2} u+1\right) * \mathcal{O}(\nabla u, N, R m)\right) .
\end{aligned}
$$

Substituting (8.42),

$$
\begin{aligned}
\partial_{t} \nabla_{\ell} N_{i j}{ }^{k}= & e^{u}\left[\nabla_{\ell} \Delta N_{i j}{ }^{k}+\left(\nabla R m+\nabla^{2} N+\nabla^{3} u\right) *(N+\nabla u)\right. \\
& \left.+\left(\nabla N+\nabla^{2} u\right) *\left(\nabla N+\nabla^{2} u\right)+\left(\nabla N+\nabla^{2} u+1\right) * \mathcal{O}(\nabla u, N, R m)\right]
\end{aligned}
$$

We can commute the derivatives $\nabla_{\ell} \Delta N_{i j}{ }^{k}$ up to lower order terms, and so

$$
\begin{aligned}
\left(\partial_{t}-e^{u} \Delta\right) \nabla_{\ell} N_{i j}{ }^{k}= & e^{u}\left[\left(\nabla R m+\nabla^{2} N+\nabla^{3} u\right) *(N+\nabla u)\right. \\
& \left.+\left(\nabla N+\nabla^{2} u\right) *\left(\nabla N+\nabla^{2} u\right)+\left(\nabla N+\nabla^{2} u+1\right) * \mathcal{O}(\nabla u, N, R m)\right]
\end{aligned}
$$

The evolution of the norm $|\nabla N|^{2}$ is

$$
\left(\partial_{t}-e^{u} \Delta\right)|\nabla N|^{2}=2\left\langle\left(\partial_{t}-e^{u} \Delta\right) \nabla N, \nabla N\right\rangle-2 e^{u}\left|\nabla^{2} N\right|^{2}+\partial_{t} g * \nabla N * \nabla N
$$

Altogether,

$$
\begin{aligned}
\left(\partial_{t}-e^{u} \Delta\right)|\nabla N|^{2} \leq & -2 e^{u}\left|\nabla^{2} N\right|^{2}+C e^{u}\left[|\nabla N|^{3}+\left|\nabla^{2} u\right||\nabla N|^{2}+\left|\nabla^{2} u\right|^{2}|\nabla N|\right. \\
& +|\nabla N|\left(|\nabla R m|+\left|\nabla^{2} N\right|+\left|\nabla^{3} u\right|\right)(|N|+|\nabla u|) \\
& \left.+\mathcal{O}(\nabla u, N, R m)\left(|\nabla N|^{2}+|\nabla N|\left|\nabla^{2} u\right|+1\right)\right] .
\end{aligned}
$$




\subsection{The evolution of the curvature tensor: proof of Theorem 6(b)}

The general formula for the variation of the curvature tensor (see e.g. [29]) is

$$
\begin{aligned}
\frac{d}{d t} R_{j i k l}= & \frac{1}{2}\left(\nabla_{i} \nabla_{k} \dot{g}_{j l}-\nabla_{i} \nabla_{l} \dot{g}_{j k}-\nabla_{j} \nabla_{k} \dot{g}_{i l}+\nabla_{j} \nabla_{l} \dot{g}_{i k}\right) \\
& +\frac{1}{2} \dot{g}_{k \lambda} R_{j i}{ }^{\lambda} l-\frac{1}{2} \dot{g}_{l \lambda} R_{j i}{ }^{\lambda} .
\end{aligned}
$$

In our case, we write the evolution of $g_{i j}$ (8.1) as

$$
\begin{aligned}
\dot{g}_{i j} & =e^{u}\left(-2 R_{i j}+2 \nabla_{i} \nabla_{j} u+E_{i j}\right), \\
E_{i j} & =-4\left(N_{-}^{2}\right)_{i j}+u_{i} u_{j}-u_{J i} u_{J j}+4 u_{p}\left(N_{i}{ }^{p}{ }_{j}+N_{j}{ }^{p}{ }_{i}\right) .
\end{aligned}
$$

Differentiating once gives

$$
\nabla_{k} \dot{g}_{j \ell}=e^{u}\left(-2 \nabla_{k} R_{j \ell}+2 \nabla_{k} \nabla_{j} \nabla_{\ell} u+\nabla_{k} E_{j \ell}\right)+e^{u}\left(-2 R_{j \ell}+2 \nabla_{j} \nabla_{\ell} u+E_{j \ell}\right) \nabla_{k} u .
$$

Differentiating twice gives

$$
\begin{aligned}
\nabla_{i} \nabla_{k} \dot{g}_{j \ell}= & e^{u}\left(-2 \nabla_{i} \nabla_{k} R_{j \ell}+2 \nabla_{i} \nabla_{k} \nabla_{j} \nabla_{\ell} u+\nabla_{i} \nabla_{k} E_{j \ell}\right) \\
& +e^{u}\left(-2 \nabla_{k} R_{j \ell}+2 \nabla_{k} \nabla_{j} \nabla_{\ell} u+\nabla_{k} E_{j \ell}\right) \nabla_{i} u+(i \leftrightarrow k) \\
& +e^{u}\left(-2 R_{j \ell}+2 \nabla_{j} \nabla_{\ell} u+E_{j \ell}\right) \nabla_{i} \nabla_{k} u+e^{u}\left(-2 R_{j \ell}+2 \nabla_{j} \nabla_{\ell} u+E_{j \ell}\right) \nabla_{k} u \nabla_{i} u .
\end{aligned}
$$

We can group this as

$$
\begin{aligned}
\nabla_{i} \nabla_{k} \dot{g}_{j \ell}= & e^{u}\left(-2 \nabla_{i} \nabla_{k} R_{j \ell}+2 \nabla_{i} \nabla_{k} \nabla_{j} \nabla_{\ell} u\right) \\
& +e^{u}\left(\nabla R m+\nabla^{3} u+\nabla^{2} N\right) * \mathcal{O}(\nabla u, N)+\nabla N *\left(\nabla^{2} u+\mathcal{O}(\nabla u, N)\right)+\nabla N * \nabla N \\
& +\mathcal{O}\left(R m, N, \nabla^{2} u, \nabla u, u\right) .
\end{aligned}
$$

We have

$$
\nabla_{i} \nabla_{k} \nabla_{j} \nabla_{\ell} u-\nabla_{i} \nabla_{j} \nabla_{k} \nabla_{\ell} u=\nabla_{i}\left(-R_{k j}{ }^{\lambda} u_{\lambda}\right)
$$

and (Lemma 7.2 in [29])

$$
\nabla_{i} \nabla_{k} R_{j \ell}-\nabla_{i} \nabla_{\ell} R_{j k}-\nabla_{j} \nabla_{k} R_{i \ell}+\nabla_{j} \nabla_{\ell} R_{i k}=-\Delta R_{j i k \ell}+R m * R m .
$$

Substituting all this into (8.71), we obtain

$$
\begin{aligned}
\left(\partial_{t}-e^{u} \Delta\right) R m= & e^{u}\left[\left(\nabla R m+\nabla^{3} u+\nabla^{2} N\right) * \mathcal{O}(\nabla u, N)+\mathcal{O}(R m, N, \nabla u)\right. \\
& \left.+\nabla N *\left(\nabla^{2} u+\mathcal{O}(\nabla u, N)\right)+\nabla N * \nabla N+\nabla^{2} u * \nabla^{2} u\right] .
\end{aligned}
$$


The norm evolves by

$$
\left(\partial_{t}-e^{u} \Delta\right)|R m|^{2}=2\left\langle\left(\partial_{t}-e^{u} \Delta\right) R m, R m\right\rangle-2 e^{u}|\nabla R m|^{2}+\partial_{t} g * R m * R m .
$$

Therefore

$$
\begin{aligned}
\left(\partial_{t}-e^{u} \Delta\right)|R m|^{2}= & e^{u}\left[-2|\nabla R m|^{2}+\left(\nabla R m+\nabla^{3} u+\nabla^{2} N\right) * \mathcal{O}(R m, \nabla u, N)\right. \\
& \left.+\left(\nabla N * \nabla N+\nabla^{2} u * \nabla^{2} u+1\right) * \mathcal{O}(R m, \nabla u, N)\right] .
\end{aligned}
$$

\subsection{Lower order estimates}

\subsubsection{Gradient estimate}

In this section, we estimate the gradient of $u$.

Proposition 5 Suppose over a finite interval $[0, T)$ the flow exists and that $|u|+|R m| \leq C$. then there exists a constant $C^{\prime}$ such that $|\nabla u|^{2} \leq C^{\prime}$ in the time interval $[0, T)$.

We recall that by our work so far, we know that $|N|^{2}$ is bounded and the $J$-invariant part of $\nabla^{2} u$ is bounded.

Equation (8.9) for the evolution of $|\nabla u|^{2}$ together with the evolution $\left(\partial_{t}-e^{u} \Delta\right) u=$ $e^{u}\left(2|\nabla u|^{2}+|N|^{2}\right)$ of $u$ imply

$$
\begin{aligned}
& \left(\partial_{t}-e^{u} \Delta\right)\left(e^{p u}|\nabla u|^{2}\right) \\
= & e^{(p+1) u}\left(-2\left|\nabla^{2} u\right|^{2}+(6-4 p)\left(\nabla^{2} u\right)_{i j} u^{i} u^{j}+8\left(N_{+}^{2}\right)_{i j} u^{i} u^{j}+2 u^{s} \nabla_{s}|N|^{2}\right. \\
& \left.+|\nabla u|^{2}\left(2 \Delta u+\left(3+2 p-p^{2}\right)|\nabla u|^{2}+(1+p)|N|^{2}\right)\right) .
\end{aligned}
$$

Let $V=e^{p u}\left(|N|^{2}+|\nabla u|^{2}\right)$ for some constant $p$. From (2.12) and (8.80), we see that

$$
\begin{aligned}
& e^{-(p+1) u}\left(\partial_{t}-e^{u} \Delta\right) V \\
= & \left(-2|\nabla N|^{2}+\left(\nabla^{2} u\right) * N^{2}+N *(R m+\nabla N) *(N+\nabla u)+N *(N+\nabla u)^{3}\right. \\
& \left.-2 p u^{s} \nabla_{s}|N|^{2}-\left(p^{2}-2 p\right)|\nabla u|^{2}|N|^{2}+p|N|^{4}\right) \\
& +\left(-2\left|\nabla^{2} u\right|^{2}+(6-4 p)\left(\nabla^{2} u\right)_{i j} u^{i} u^{j}+8\left(N_{+}^{2}\right)_{i j} u^{i} u^{j}+2 u^{s} \nabla_{s}|N|^{2}\right. \\
& \left.+|\nabla u|^{2}\left(2 \Delta u+\left(3+2 p-p^{2}\right)|\nabla u|^{2}+(1+p)|N|^{2}\right)\right) .
\end{aligned}
$$


We note that $\left(\nabla^{2} u\right) * N^{2}$ represent terms of the form $a\left(\nabla^{2} u\right)^{i j}\left(N_{+}^{2}\right)_{i j}+b\left(\nabla^{2} u\right)^{i j}\left(N_{-}^{2}\right)_{i j}$ for some constant $a$ and $b$. Those terms are also bounded since $N_{ \pm}^{2}$ is $J$-invariant hence only the $J$-invariant part of $\nabla^{2} u$ contributes to this term, which is bounded. Also, we can control all the terms linear in $\nabla N$ by the good term $-|\nabla N|^{2}$. Therefore

$$
\begin{aligned}
& e^{-(p+1) u}\left(\partial_{t}-e^{u} \Delta\right) V \\
\leq & -2\left|\nabla^{2} u\right|^{2}+(6-4 p)\left(\nabla^{2} u\right)_{i j} u^{i} u^{j}+\left(3+2 p-p^{2}\right)|\nabla u|^{4} \\
& +C(p)|\nabla u|^{3}+C(p) .
\end{aligned}
$$

To handle the term $\left(\nabla^{2} u\right)_{i j} u^{i} u^{j}$, we need to make use of the fact that the $J$-invariant part of $\nabla^{2} u$ is bounded. To do so, let us denote the $J$-invariant and the $J$-anti-invariant parts of $\nabla^{2} u$ by $\nabla_{J}^{2} u$ and $\nabla_{-J}^{2} u$ respectively. Under this notation, we see that

$$
\begin{aligned}
& -2\left|\nabla^{2} u\right|^{2}+(6-4 p)\left(\nabla^{2} u\right)_{i j} u^{i} u^{j}+\left(3+2 p-p^{2}\right)|\nabla u|^{4} \\
= & -2\left|\nabla_{J}^{2} u\right|^{2}-2\left|\nabla_{-J}^{2} u\right|^{2}+(6-4 p)\left(\nabla_{J}^{2} u+\nabla_{-J}^{2} u, \nabla u \otimes \nabla u\right)+\left(3+2 p-p^{2}\right)|\nabla u|^{4} \\
\leq & -2\left|\nabla_{-J}^{2} u\right|^{2}+(6-4 p)\left(\nabla_{-J}^{2} u, \nabla u \otimes \nabla u\right)+\left(3+2 p-p^{2}\right)|\nabla u|^{4}+C|\nabla u|^{2} .
\end{aligned}
$$

The advantage of this consideration is that only the $J$-anti-invariant part of $\nabla u \otimes \nabla u$, namely $\frac{1}{2}\left(u_{i} u_{j}-u_{J i} u_{J j}\right)$, contributes to the inner product term. Therefore

$$
\begin{aligned}
& -2\left|\nabla^{2} u\right|^{2}+(6-4 p)\left(\nabla^{2} u\right)_{i j} u^{i} u^{j}+\left(3+2 p-p^{2}\right)|\nabla u|^{4} \\
\leq & -2\left|\nabla_{-J}^{2} u\right|^{2}+(3-2 p)\left(\nabla_{-J}^{2} u\right)^{i j}\left(u_{i} u_{j}-u_{J i} u_{J j}\right)+\left(3+2 p-p^{2}\right)|\nabla u|^{4}+C|\nabla u|^{2} \\
= & -2\left|\left(\nabla_{-J}^{2} u\right)_{i j}+\frac{1}{2}\left(p-\frac{3}{2}\right)\left(u_{i} u_{j}-u_{J i} u_{J j}\right)\right|^{2}+\frac{1}{2}\left(p-\frac{3}{2}\right)^{2}\left|u_{i} u_{j}-u_{J i} u_{J j}\right|^{2} \\
& +\left(3+2 p-p^{2}\right)|\nabla u|^{4}+C|\nabla u|^{2} \\
\leq & \frac{1}{2}\left(p-\frac{3}{2}\right)^{2}\left|u_{i} u_{j}-u_{J i} u_{J j}\right|^{2}+\left(3+2 p-p^{2}\right)|\nabla u|^{4}+C|\nabla u|^{2} .
\end{aligned}
$$

The $J$-anti-invariant part of $\nabla u \otimes \nabla u$ has half of the norm square compared to the full $\nabla u \otimes \nabla u$ :

$$
\left|u_{i} u_{j}-u_{J i} u_{J j}\right|^{2}=2|\nabla u \otimes \nabla u|^{2}=2|\nabla u|^{4} .
$$

So the conclusion is that

$$
\begin{aligned}
& -2\left|\nabla^{2} u\right|^{2}+(2-4 p)\left(\nabla^{2} u\right)_{i j} u^{i} u^{j}+\left(3+2 p-p^{2}\right)|\nabla u|^{4} \\
\leq & \left(\left(p-\frac{3}{2}\right)^{2}-p^{2}+2 p+3\right)|\nabla u|^{4}+C|\nabla u|^{2} \\
= & \left(-p+\frac{9}{4}+3\right)|\nabla u|^{4}+C|\nabla u|^{2} \\
\leq & -|\nabla u|^{4}+C|\nabla u|^{2}
\end{aligned}
$$

for $p=(9 / 4)+4$. Thus

$$
e^{-(p+1) u}\left(\partial_{t}-e^{u} \Delta\right) V \leq-|\nabla u|^{4}+C(p)|\nabla u|^{3}+C(p) .
$$

Then by maximum principle and the boundedness of $u$, we prove the proposition. 


\subsubsection{Second order estimate}

In this section, we obtain estimates on $|\nabla N|+\left|\nabla^{2} u\right|$. We refer to $\nabla N$ and $\nabla^{2} u$ as second order terms since they involve two derivatives of $\varphi$.

Proposition 6 Let $\left(g_{i j}(t), u(t)\right)$ evolve by Type IIA flow on $M \times[0, T]$. Suppose

$$
\sup _{M \times[0, T]}(|R m|+|N|+|\nabla u|+|u|) \leq \Lambda .
$$

Then there exists a constant $C$ depending on $\Lambda$ and $\left(g_{i j}(0), u(0)\right)$ such that

$$
\sup _{M \times[0, T]}\left(|\nabla N|+\left|\nabla^{2} u\right|\right) \leq C .
$$

A basic building block in the construction of our test function for this estimate will be

$$
\tau(z)=|N|^{2}+|\nabla u|^{2} .
$$

It satisfies $\tau \leq C$, and using our work so far, its evolution can be estimated by

$$
\left(\partial_{t}-e^{u} \Delta\right) \tau \leq-|\nabla N|^{2}-\left|\nabla^{2} u\right|^{2}+C .
$$

We start with the test function

$$
Q=\frac{\left|\nabla^{2} u\right|^{2}+|\nabla N|^{2}}{K-\tau}
$$

where $K$ is a large constant to be determined. We can compute its evolution

$$
\begin{aligned}
\left(\partial_{t}-e^{u} \Delta\right) Q= & \frac{1}{K-\tau}\left(\partial_{t}-e^{u} \Delta\right)\left(\left|\nabla^{2} u\right|^{2}+|\nabla N|^{2}\right)+\frac{\left|\nabla^{2} u\right|^{2}+|\nabla N|^{2}}{(K-\tau)^{2}}\left(\partial_{t}-e^{u} \Delta\right) \tau \\
& -\frac{2 e^{u}}{(K-\tau)^{2}} \nabla_{i}\left(\left|\nabla^{2} u\right|^{2}+|\nabla N|^{2}\right) \nabla^{i} \tau-2 e^{u} \frac{\left|\nabla^{2} u\right|^{2}+|\nabla N|^{2}}{(K-\tau)^{3}}|\nabla \tau|^{2} .
\end{aligned}
$$

By our evolution equations (8.70), (8.16), (8.88) for $|\nabla N|^{2},\left|\nabla^{2} u\right|^{2}$, and $\tau$, we obtain the estimate

$$
\begin{aligned}
\left(\partial_{t}-e^{u} \Delta\right) Q \leq & \frac{e^{u}}{(K-\tau)}\left[-\left|\nabla^{3} u\right|^{2}-\left|\nabla^{2} N\right|^{2}+C|\nabla N|^{3}+C\left|\nabla^{2} u\right|^{3}\right. \\
& +C|\nabla R m||\nabla N|+C|\nabla R m|\left|\nabla^{2} u\right|+C|\nabla N|\left|\nabla^{3} u\right| \\
& +C|\nabla N|^{2}\left|\nabla^{2} u\right|+C\left|\nabla^{2} N\right|\left|\nabla^{2} u\right|+C \\
& -\frac{|\nabla N|^{4}}{(K-\tau)}-\frac{\left|\nabla^{2} u\right|^{4}}{(K-\tau)}-2 \frac{\left|\nabla^{2} u\right|^{2}|\nabla N|^{2}}{(K-\tau)} \\
& +C \frac{\left|\nabla^{2} u\right|^{2}+|\nabla N|^{2}}{(K-\tau)} \\
& \left.-\frac{2}{(K-\tau)} \nabla_{i}\left(\left|\nabla^{2} u\right|^{2}+|\nabla N|^{2}\right) \nabla^{i} \tau-2 \frac{\left|\nabla^{2} u\right|^{2}+|\nabla N|^{2}}{(K-\tau)^{2}}|\nabla \tau|^{2}\right]
\end{aligned}
$$


By the bound on $|N|$ and $|\nabla u|$, we can choose $K-\tau \geq \frac{K}{2}$ large. The terms $|\nabla N|^{4}$ and $\left|\nabla^{2} u\right|^{4}$ can absorb lower order terms. We also drop the last term.

$$
\begin{aligned}
\left(\partial_{t}-e^{u} \Delta\right) Q \leq & \frac{e^{u}}{(K-\tau)}\left[-\left|\nabla^{3} u\right|^{2}-\left|\nabla^{2} N\right|^{2}+\frac{1}{10}|\nabla R m|^{2}+C(K)\right. \\
& -\frac{|\nabla N|^{4}}{2 K}-\frac{\left|\nabla^{2} u\right|^{4}}{2 K}-\frac{\left|\nabla^{2} u\right|^{2}|\nabla N|^{2}}{K} \\
& \left.-\frac{2}{(K-\tau)} \nabla_{i}\left(\left|\nabla^{2} u\right|^{2}+|\nabla N|^{2}\right) \nabla^{i} \tau\right]
\end{aligned}
$$

Using $|\nabla \tau| \leq C\left(\left|\nabla^{2} u\right|+|\nabla N|+1\right)$, we can estimate

$$
\begin{aligned}
& -\frac{2}{(K-\tau)} \nabla_{i}\left(\left|\nabla^{2} u\right|^{2}+|\nabla N|^{2}\right) \nabla^{i} \tau \\
\leq & \left.\frac{C}{K}|\nabla| \nabla^{2} u\right|^{2}+\nabla|\nabla N|^{2}|| \nabla \tau \mid \\
\leq & \frac{C}{K}\left(\left|\nabla^{3} u\right|\left(\left|\nabla^{2} u\right|^{2}+\left|\nabla^{2} u\right||\nabla N|\right)+\left|\nabla^{2} N\right|\left(|\nabla N|^{2}+\left|\nabla^{2} u\right||\nabla N|\right)\right) \\
\leq & \frac{1}{2}\left|\nabla^{3} u\right|^{2}+\frac{1}{2}\left|\nabla^{2} N\right|^{2}+\frac{C_{0}}{K^{2}}\left|\nabla^{2} u\right|^{4}+\frac{C_{0}}{K^{2}}\left|\nabla^{2} u\right|^{2}|\nabla N|^{2}+\frac{C_{0}}{K^{2}}|\nabla N|^{4}
\end{aligned}
$$

Choose $K$ large such that $K \geq 4 C_{0} \gg 1$. Then the main inequality becomes

$$
\begin{aligned}
\left(\partial_{t}-e^{u} \Delta\right) Q \leq & \frac{e^{u}}{(K-\tau)}\left[-\frac{1}{2}\left|\nabla^{3} u\right|^{2}-\frac{1}{2}\left|\nabla^{2} N\right|^{2}-\frac{|\nabla N|^{4}}{4 K}-\frac{\left|\nabla^{2} u\right|^{4}}{4 K}\right. \\
& \left.+\frac{1}{10}|\nabla R m|^{2}+C(K)\right] .
\end{aligned}
$$

We can now prove that if $|u|+|\nabla u|+|N|+|R m| \leq C$ along the flow, then we can bound $|\nabla N|$ and $\left|\nabla^{2} u\right|$. Consider the test function

$$
S=Q+|R m|^{2}
$$

By (8.92) and (2.13), we can estimate the evolution of $Q$ and $|R m|^{2}$.

$$
\begin{aligned}
\left(\partial_{t}-e^{u} \Delta\right) S \leq & \frac{e^{u}}{(K-\tau)}\left[-\frac{1}{2}\left|\nabla^{3} u\right|^{2}-\frac{1}{2}\left|\nabla^{2} N\right|^{2}-\frac{|\nabla N|^{4}}{4 K}-\frac{\left|\nabla^{2} u\right|^{4}}{4 K}\right. \\
& \left.+\frac{1}{10}|\nabla R m|^{2}+C(K)\right]-e^{u}|\nabla R m|^{2} \\
& +C\left|\nabla^{3} u\right|+C\left|\nabla^{2} N\right|+C|\nabla N|^{2}+C\left|\nabla^{2} u\right|^{2}+C
\end{aligned}
$$

As long as $K$ is large enough such that $K-\tau \geq 1$, it follows that at a maximum point $\left(x_{0}, t_{0}\right)$ of $S$ with $t_{0}>0$, then

$$
|\nabla N|^{2}\left(x_{0}, t_{0}\right)+\left|\nabla^{2} u\right|^{2}\left(x_{0}, t_{0}\right) \leq C(K) .
$$


Since $Q=\frac{\left|\nabla^{2} u\right|^{2}+|\nabla N|^{2}}{K-\tau}$, it follows that $Q\left(x_{0}, t_{0}\right) \leq C$ and hence $S\left(x_{0}, t_{0}\right) \leq C$.

Therefore $S$ is bounded on $M \times[0, T]$. It follows that if $|u|+|\nabla u|+|N|+|R m| \leq C_{0}$ on $M \times[0, T]$, then

$$
|\nabla N|+\left|\nabla^{2} u\right| \leq C
$$

where $C$ depends on $C_{0}$ and the initial data.

\subsection{Higher order estimates}

In this section, we prove the following estimate.

Proposition 7 Let $\left(g_{i j}(t), u(t)\right)$ evolve by Type IIA flow on $M \times[0, T]$. Suppose

$$
\sup _{M \times[0, T]}\left(|R m|+|\nabla N|+|N|+\left|\nabla^{2} u\right|+|\nabla u|+|u|\right) \leq \Lambda .
$$

Then for each integer $k \geq 1$, there exists a constant $C_{k}$ depending on $k, \Lambda$ and $\left(g_{i j}(0), u(0)\right)$ such that

$$
\sup _{M \times[0, T]}\left(\left|\nabla^{k} R m\right|+\left|\nabla^{k+1} N\right|+\left|\nabla^{k+2} u\right|\right) \leq C_{k} .
$$

Note: in earlier work, we showed that the estimate $|u|+|R m| \leq C$ implies the estimate $|\nabla u|+\left|\nabla^{2} u\right|+|N|+|\nabla N| \leq C$. Combining these two results, we conclude that if $|u|+$ $|R m| \leq C$ remains bounded along the flow, then all geometric terms remain bounded.

Let $I_{k}$ denote any combination of geometric terms of derivative order $\leq k$ in the metric. For example,

$$
\begin{aligned}
& I_{2}=f\left(u, \nabla u, \nabla^{2} u, N, \nabla N, R m\right), \\
& I_{3}=f\left(u, \nabla u, \nabla^{2} u, \nabla^{3} u, N, \nabla N, \nabla^{2} N, R m, \nabla R m\right) .
\end{aligned}
$$

In this section, we will evolve all higher order geometric terms appearing in the equation of the metric Type IIA flow.

\subsubsection{The evolution of $\left|\nabla^{k} R m\right|^{2}$}

We write the evolution of the curvature as

$$
\left(\partial_{t}-e^{u} \Delta\right) R m=E(R m)
$$

where

$$
E(R m)=\left(\nabla^{3} u+\nabla^{2} N+\nabla R m\right) * I_{1}+I_{2} .
$$

We have for example

$$
\nabla E(R m)=\left(\nabla^{4} u+\nabla^{3} N+\nabla^{2} R m\right) * I_{1}+\left(\nabla^{3} u+\nabla^{2} N+\nabla R m\right) * I_{2}+I_{2}
$$


and in general

$$
\begin{aligned}
\nabla^{k} E(R m)= & \left(\nabla^{k+3} u+\nabla^{k+2} N+\nabla^{k+1} R m\right) * I_{1} \\
& +\left(\nabla^{k+2} u+\nabla^{k+3} N+\nabla^{k+1} R m\right) * I_{2}+I_{k+1}
\end{aligned}
$$

Then

$$
\begin{aligned}
\partial_{t} \nabla^{k} R m & =\partial_{t}(\partial+\Gamma)^{k} R m \\
& =\nabla^{k}\left(\partial_{t} R m\right)+\sum_{i=0}^{k-1} \nabla^{i} \partial_{t} \Gamma \nabla^{k-1-i} R m \\
& =\nabla^{k} E(R m)+\nabla^{k}\left(e^{u} \Delta R m\right)+\sum_{i=0}^{k-1} \nabla^{i} \partial_{t} \Gamma \nabla^{k-1-i} R m
\end{aligned}
$$

We have the general commutator formula

$$
\nabla^{k} \Delta A=\Delta \nabla^{k} A+\nabla^{k}(R m * A)
$$

which implies

$$
\begin{aligned}
\left(\partial_{t}-e^{u} \Delta\right) \nabla^{k} R m= & \nabla^{k}(R m * R m)+\nabla^{k} E(R m)+\sum_{i=1}^{k} \nabla^{i} e^{u} * \nabla^{k-i} \Delta R m \\
& +\sum_{i=0}^{k-1} \nabla^{i} \partial_{t} \Gamma * \nabla^{k-1-i} R m
\end{aligned}
$$

We note

$$
\partial_{t} \Gamma=\left(\nabla^{3} u+\nabla^{2} N+\nabla R m\right) * I_{1}+I_{2},
$$

and

$$
\begin{aligned}
\nabla^{k} \partial_{t} \Gamma= & \left(\nabla^{k+3} u+\nabla^{k+2} N+\nabla^{k+1} R m\right) * I_{1} \\
& +\left(\nabla^{k+2} u+\nabla^{k+3} N+\nabla^{k+1} R m\right) * I_{2}+I_{k+1} .
\end{aligned}
$$

Therefore

$$
\begin{aligned}
\left(\partial_{t}-e^{u} \Delta\right) \nabla^{k} R m= & I_{1} *\left(\nabla^{k+3} u+\nabla^{k+2} N+\nabla^{k+1} R m\right) \\
& +I_{2} *\left(\nabla^{k+2} u+\nabla^{k+1} N+\nabla^{k} R m\right)+I_{k+1}
\end{aligned}
$$

The norm is evolving by

$$
\begin{aligned}
\left(\partial_{t}-e^{u} \Delta\right)\left|\nabla^{k} R m\right|^{2}= & 2\left\langle\left(\partial_{t}-e^{u} \Delta\right) \nabla^{k} R m, \nabla^{k} R m\right\rangle \\
& -2 e^{u}\left|\nabla^{k+1} R m\right|^{2}+\partial_{t} g * \nabla^{k} R m * \nabla^{k} R m .
\end{aligned}
$$

Thus

$$
\begin{aligned}
\left(\partial_{t}-e^{u} \Delta\right)\left|\nabla^{k} R m\right|^{2}= & -2 e^{u}\left|\nabla^{k+1} R m\right|^{2} \\
& +I_{1} *\left(\nabla^{k+3} u+\nabla^{k+2} N+\nabla^{k+1} R m\right) * \nabla^{k} R m \\
& +I_{2} *\left(\nabla^{k+2} u+\nabla^{k+1} N+\nabla^{k} R m\right)^{2}+I_{k+1} * \nabla^{k} R m .(8.111)
\end{aligned}
$$




\subsubsection{The evolution of $\left|\nabla^{k} N\right|^{2}$}

We will evolve $\nabla^{k} N$ in this section for all $k \geq 2$. We write $\left(\partial_{t}-e^{u} \Delta\right) N=E(N)$. Higher order terms evolve by

$$
\begin{aligned}
\partial_{t} \nabla^{k} N= & \partial_{t}(\partial+\Gamma)^{k} N \\
= & \nabla^{k}\left(\partial_{t} N\right)+\sum_{i=0}^{k-1} \nabla^{i} \partial_{t} \Gamma * \nabla^{k-1-i} N \\
= & \nabla^{k} E(N)+e^{u} \nabla^{k} \Delta N+\sum_{i=1}^{k} \nabla^{i} e^{u} * \nabla^{k-i} \Delta N \\
& +\sum_{i=0}^{k-1} \nabla^{i} \partial_{t} \Gamma * \nabla^{k-1-i} N .
\end{aligned}
$$

Using (8.105) to commute derivatives gives

$$
\begin{aligned}
\left(\partial_{t}-e^{u} \Delta\right) \nabla^{k} N= & \nabla^{k} E(N)+\sum_{i=1}^{k} \nabla^{i} e^{u} * \nabla^{k-i} \Delta N \\
& +\nabla^{k}(R m * N)+\sum_{i=0}^{k-1} \nabla^{i} \partial_{t} \Gamma * \nabla^{k-1-i} N .
\end{aligned}
$$

By (8.42),

$$
E(N)=\left(\nabla^{2} u+\nabla N+R m\right) * I_{1}+I_{1}
$$

Differentiating this once gives

$$
\nabla E(N)=\left(\nabla^{3} u+\nabla^{2} N+\nabla R m\right) * I_{1}+I_{2}
$$

Differentiating again, we obtain

$$
\begin{aligned}
\nabla^{2} E(N)= & \left(\nabla^{4} u+\nabla^{3} N+\nabla^{2} R m\right) * I_{1} \\
& +\left(\nabla^{3} u+\nabla^{2} N+\nabla R m\right) * I_{2}+I_{2} .
\end{aligned}
$$

Higher order derivatives are

$$
\begin{aligned}
\nabla^{k} E(N)= & \left(\nabla^{k+2} u+\nabla^{k+1} N+\nabla^{k} R m\right) * I_{1} \\
& +\left(\nabla^{k+1} u+\nabla^{k} N+\nabla^{k-1} R m\right) * I_{2}+I_{k}
\end{aligned}
$$

for $k \geq 2$. Substituting this and (8.107) into (8.113)

$$
\begin{aligned}
\left(\partial_{t}-e^{u} \Delta\right) \nabla^{k} N= & \left(\nabla^{k+2} u+\nabla^{k+1} N+\nabla^{k} R m\right) * I_{1} \\
& +\left(\nabla^{k+1} u+\nabla^{k} N+\nabla^{k-1} R m\right) * I_{2}+I_{k} .
\end{aligned}
$$


The norm evolves by

$$
\begin{aligned}
\left(\partial_{t}-e^{u} \Delta\right)\left|\nabla^{k} N\right|^{2}= & -2 e^{u}\left|\nabla^{k+1} N\right|^{2}+2\left\langle\left(\partial_{t}-e^{u} \Delta\right) \nabla^{k} N, \nabla^{k} N\right\rangle \\
& +\partial_{t} g * \nabla^{k} N * \nabla^{k} N .
\end{aligned}
$$

Therefore

$$
\begin{aligned}
\left(\partial_{t}-e^{u} \Delta\right)\left|\nabla^{k} N\right|^{2}= & -2 e^{u}\left|\nabla^{k+1} N\right|^{2}+I_{1} *\left(\nabla^{k+2} u+\nabla^{k+1} N+\nabla^{k} R m\right) * \nabla^{k} N \\
& +I_{2} *\left(\nabla^{k+1} u+\nabla^{k} N+\nabla^{k-1} R m\right)^{2}+I_{k} * \nabla^{k} N .
\end{aligned}
$$

\subsubsection{The evolution of $\left|\nabla^{k} u\right|^{2}$}

Denote as before $\left(\partial_{t}-e^{u} \Delta\right) u=E(u)$. We will compute the evolution of $\nabla^{k} u$ for $k \geq 3$.

$$
\begin{aligned}
\partial_{t} \nabla^{k} u= & \partial_{t}(\partial+\Gamma)^{k-1} \partial u \\
= & \nabla^{k}\left(\partial_{t} u\right)+\sum_{i=0}^{k-2} \nabla^{i} \partial_{t} \Gamma * \nabla^{k-1-i} u \\
= & e^{u} \nabla^{k} \Delta u+\nabla^{k} E(u)+\sum_{i=1}^{k} \nabla^{i} e^{u} \nabla^{k-i} \Delta u+\sum_{i=0}^{k-2} \nabla^{i} \partial_{t} \Gamma * \nabla^{k-1-i} u \\
= & e^{u} \Delta \nabla^{k} u+\sum_{i=0}^{k-1} \nabla^{i} R m * \nabla^{k-i} u+\nabla^{k} E(u) \\
& +\sum_{i=1}^{k} \nabla^{i} e^{u} \nabla^{k-i} \Delta u+\sum_{i=0}^{k-2} \nabla^{i} \partial_{t} \Gamma * \nabla^{k-1-i} u
\end{aligned}
$$

The evolution of $u$ is of the form $E(u)=I_{1}$. We will differentiate this 3 times before it becomes linear enough to use in our general argument. Differentiating once

$$
\nabla E(u)=\left(\nabla^{2} u+\nabla N+R m\right) * I_{1}+I_{1},
$$

twice

$$
\nabla^{2} E(u)=\left(\nabla^{3} u+\nabla^{2} N+\nabla R m\right) * I_{1}+I_{2}
$$

and three times

$$
\nabla^{3} E(u)=\left(\nabla^{4} u+\nabla^{3} N+\nabla^{2} R m\right) * I_{1}+\left(\nabla^{3} u+\nabla^{2} N+\nabla R m\right) * I_{2}+I_{2} .
$$

Higher order derivatives are

$$
\nabla^{k} E(u)=\left(\nabla^{k+1} u+\nabla^{k} N+\nabla^{k-1} R m\right) * I_{1}+\left(\nabla^{k} u+\nabla^{k-1} N+\nabla^{k-2} R m\right) * I_{2}+I_{k-1} .
$$

for $k \geq 3$. Substituting this and (8.107) into (8.121)

$$
\begin{aligned}
\left(\partial_{t}-e^{u} \Delta\right) \nabla^{k} u= & \left(\nabla^{k+1} u+\nabla^{k} N+\nabla^{k-1} R m\right) * I_{1} \\
& +\left(\nabla^{k} u+\nabla^{k-1} N+\nabla^{k-2} R m\right) * I_{2}+I_{k-1}
\end{aligned}
$$


Using the evolution of the norm

$$
\begin{aligned}
\left(\partial_{t}-e^{u} \Delta\right)\left|\nabla^{k} u\right|^{2}= & -2 e^{u}\left|\nabla^{k+1} u\right|^{2}+2\left\langle\left(\partial_{t}-e^{u} \Delta\right) \nabla^{k} u, \nabla^{k} u\right\rangle \\
& +\partial_{t} g * \nabla^{k} u * \nabla^{k} u
\end{aligned}
$$

we conclude

$$
\begin{aligned}
\left(\partial_{t}-e^{u} \Delta\right)\left|\nabla^{k} u\right|^{2}= & -2 e^{u}\left|\nabla^{k+1} u\right|^{2}+I_{1} *\left(\nabla^{k+1} u+\nabla^{k} N+\nabla^{k-1} R m\right) * \nabla^{k} u \\
& +I_{2} *\left(\nabla^{k} u+\nabla^{k-1} N+\nabla^{k-2} R m\right)^{2}+I_{k-1} * \nabla^{k} u .
\end{aligned}
$$

\subsubsection{Estimates: proof of Theorem 7}

Putting everything together, we obtain

$$
\begin{aligned}
& \left(\partial_{t}-e^{u} \Delta\right)\left(\left|\nabla^{k} u\right|^{2}+\left|\nabla^{k-1} N\right|^{2}+\left|\nabla^{k-2} R m\right|^{2}\right) \\
= & -2 e^{u}\left(\left|\nabla^{k+1} u\right|^{2}+\left|\nabla^{k} N\right|^{2}+\left|\nabla^{k-1} R m\right|^{2}\right) \\
& +I_{1} *\left(\nabla^{k+1} u+\nabla^{k} N+\nabla^{k-1} R m\right) *\left(\nabla^{k} u+\nabla^{k-1} N+\nabla^{k-2} R m\right) \\
& +I_{2} *\left(\nabla^{k} u+\nabla^{k-1} N+\nabla^{k-2} R m\right)^{2}+I_{k-1} *\left(\nabla^{k} u+\nabla^{k-1} N+\nabla^{k-2} R m\right)(8.129)
\end{aligned}
$$

Let $k \geq 3$. Suppose $I_{k-1} \leq C$. Then

$$
\begin{aligned}
& \left(\partial_{t}-e^{u} \Delta\right)\left(\left|\nabla^{k} u\right|^{2}+\left|\nabla^{k-1} N\right|^{2}+\left|\nabla^{k-2} R m\right|^{2}\right) \\
\leq & -e^{u}\left(\left|\nabla^{k+1} u\right|^{2}+\left|\nabla^{k} N\right|^{2}+\left|\nabla^{k-1} R m\right|^{2}\right) \\
& +C\left|\nabla^{k} u\right|^{2}+C\left|\nabla^{k-1} N\right|^{2}+C\left|\nabla^{k-2} R m\right|^{2}+C
\end{aligned}
$$

and

$$
\begin{aligned}
& \left(\partial_{t}-e^{u} \Delta\right)\left(\left|\nabla^{k-1} u\right|^{2}+\left|\nabla^{k-2} N\right|^{2}+\left|\nabla^{k-3} R m\right|^{2}\right) \\
\leq & -e^{u}\left(\left|\nabla^{k} u\right|^{2}+\left|\nabla^{k-1} N\right|^{2}+\left|\nabla^{k-2} R m\right|^{2}\right)+C .
\end{aligned}
$$

It follows that the test function

$$
\begin{aligned}
& \left(\partial_{t}-e^{u} \Delta\right)\left[\left|\nabla^{k} u\right|^{2}+\left|\nabla^{k-1} N\right|^{2}+\left|\nabla^{k-2} R m\right|^{2}+\Lambda\left(\left|\nabla^{k-1} u\right|^{2}+\left|\nabla^{k-2} N\right|^{2}+\left|\nabla^{k-3} R m\right|^{2}\right)\right] \\
\leq & -\left|\nabla^{k} u\right|^{2}-\left|\nabla^{k-1} N\right|^{2}-\left|\nabla^{k-2} R m\right|^{2}+\Lambda C
\end{aligned}
$$

for $\Lambda \gg 1$ large. By the maximum principle, we conclude that if $I_{k-1} \leq K$ then

$$
\left|\nabla^{k} u\right|^{2}+\left|\nabla^{k-1} N\right|^{2}+\left|\nabla^{k-2} R m\right|^{2} \leq C(K, g(0))
$$

and hence $I_{k}$ is bounded along the flow. This argument shows that if $I_{2}$ is bounded, then $I_{k}$ is bounded for all $k$. 


\subsection{Long-time existence}

Let $(u(t), g(t))$ be a solution to the Type IIA flow on $[0, T)$. Suppose $|u|+|R m| \leq C$ on $M \times[0, T)$. We have shown that in this case $\left|\nabla^{k} u\right|+\left|\nabla^{k} N\right|+\left|\nabla^{k} R m\right| \leq C$ for all $k \geq 1$. We now give the standard argument (see e.g. [29]) which shows that the flow can be extended past $t=T$. Denote $\partial_{t} g_{i j}=E_{i j}$. Our estimates imply that

$$
|E|+\left|\nabla^{k} E\right| \leq C
$$

where $\nabla$ is with respect to the evolving metric $g(t)$. If we take $x \in M, v \in T_{x} M$ and $t_{1}, t_{2} \in(0, T)$, then

$$
\left|\log g\left(t_{2}\right)(v, v)-\log g\left(t_{1}\right)(v, v)\right|=\left|\int_{t_{1}}^{t_{2}} \frac{\dot{g}(\tau)(v, v)}{g(\tau)(v, v)} d \tau\right| \leq C\left|t_{2}-t_{1}\right| .
$$

It follows that $g(t)$ is a Cauchy sequence as $t \rightarrow T$ and

$$
e^{-C T} g(0) \leq g(t) \leq e^{C T} g(0)
$$

and the metrics $g_{i j}$ do not degenerate on $[0, T)$. Let $\bar{\nabla}$ denote the covariant derivative with respect to $\bar{g}=g(0)$. We have

$$
\partial_{t} \bar{\nabla}_{k} g_{i j}=\bar{\nabla}_{k} \dot{g}_{i j}=\nabla_{k} E_{i j}+(\bar{\Gamma}-\Gamma) * E_{i j}
$$

The difference between two connections is

$$
\Gamma_{i j}^{k}-\bar{\Gamma}_{i j}^{k}=\frac{1}{2} g^{k p}\left(-\bar{\nabla}_{p} g_{i j}+\bar{\nabla}_{i} g_{p j}+\bar{\nabla}_{j} g_{p i}\right),
$$

and hence

$$
\partial_{t} \bar{\nabla} g=\bar{\nabla} g * E+\mathcal{O}(1)
$$

Therefore

$$
\partial_{t}|\bar{\nabla} g|_{\bar{g}}^{2} \leq C|\bar{\nabla} g|_{\bar{g}}^{2}+C|\bar{\nabla} g|_{\hat{g}}
$$

and hence $|\bar{\nabla} g|_{\bar{g}} \leq C(T)$ on $[0, T)$. Higher order derivatives are similar: indeed, let $k \geq 1$ and suppose that $\left|\bar{\nabla}^{\ell} g\right|_{\bar{g}} \leq C$ for all $\ell \leq k$. Then a similar calculation gives

$$
\partial_{t} \bar{\nabla}^{k+1} g=\bar{\nabla}^{k+1} g * E+\mathcal{O}(1),
$$

from which it follows that

$$
\left|\bar{\nabla}^{k+1} g\right|_{\bar{g}} \leq C(T) .
$$

Therefore, the evolving metrics $g_{i j}$ and all their derivatives are bounded on $[0, T)$. Since we showed $g(t)$ is Cauchy, it follows that $g(t) \rightarrow g(T)$ smoothly as $t \rightarrow T$. A similar argument shows that $u(t) \rightarrow u(T)$. 
This produces a limiting pair $(g(T), u(T))$. The linear ODE for $\varphi$ given in Theorem 4 has coefficients which only depend on $\tilde{g}_{i j}=e^{u} g_{i j}$, thus these coefficients are smoothly defined on $[0, T]$. It follows that $\varphi(t)$ has a smooth solution on $[0, T]$. By the nondegeneracy estimate (7.25), we have that $\varphi(T)$ is closed, primitive, and in the positive cone $\left(-\lambda_{\varphi}\right)>0$. By the short-time existence theorem, the flow can be extended to $[0, T+\epsilon)$ for some $\epsilon>0$. The discussion here also implies that $\left|\nabla^{\alpha} \varphi\right| \leq C$ on $[0, T)$ for any multi-index $\alpha$. This completes the proof of Theorem 7 .

\section{Examples and Applications}

In this section, we discuss a range of examples and applications of the Type IIA flow with no sources.

\subsection{The stationary points: proof of Theorem 8}

We begin with the proof of Theorem 8. First, we note that $-d^{\Lambda}\left(|\varphi|^{2} \hat{\varphi}\right)=\Lambda d\left(|\varphi|^{2} \hat{\varphi}\right)=$ $\partial_{-}\left(|\varphi|^{2} \hat{\varphi}\right)$, where $\partial_{-}$is a first order differential operator introduced in [50] such that

$$
d\left(|\varphi|^{2} \hat{\varphi}\right)=\omega \wedge \partial_{-}\left(|\varphi|^{2} \hat{\varphi}\right)
$$

and $\partial_{-}\left(|\varphi|^{2} \hat{\varphi}\right)$ is a primitive 2 -form. Therefore the stationary point equation $d \Lambda d\left(|\varphi|^{2} \star\right.$ $\varphi)=0$ can be expressed as $d \partial_{-}\left(|\varphi|^{2} \hat{\varphi}\right)=0$. In particular,

$$
0=\int_{M} d \partial_{-}\left(|\varphi|^{2} \hat{\varphi}\right) \wedge \hat{\varphi}=-\int_{M} \partial_{-}\left(|\varphi|^{2} \hat{\varphi}\right) \wedge d \hat{\varphi} .
$$

Combining (6.40), (6.30), and $\beta=0$ implies $d \hat{\varphi}=\alpha \wedge \hat{\varphi}+\tilde{\mathfrak{T}} \otimes \hat{\varphi}$. By (6.43), we have $\tilde{\mathfrak{T}}=N-\frac{1}{4}\left(d^{c} \tilde{\omega}+\mathcal{M}\left(d^{c} \tilde{\omega}\right)\right)$. By Lemma 15, we see that

$$
d \hat{\varphi}=N \otimes \hat{\varphi}
$$

which is a $(2,2)$-form (by the argument in the proof of Lemma 14). Therefore

$$
d\left(|\varphi|^{2} \hat{\varphi}\right)=|\varphi|^{2}(-\alpha \wedge \hat{\varphi}+N \otimes \hat{\varphi}),
$$

where the first term is a $(3,1)+(1,3)$-form, the second is a $(2,2)$-form, and $\alpha=-d|\varphi|^{2}$. It follows that

$$
\begin{aligned}
\partial_{-} \hat{\varphi} & =\Lambda(N \otimes \hat{\varphi}), \\
\partial_{-}\left(|\varphi|^{2} \hat{\varphi}\right) & =|\varphi|^{2}\left(-\Lambda(\alpha \wedge \hat{\varphi})+\partial_{-} \hat{\varphi}\right),
\end{aligned}
$$

where $\Lambda(N \otimes \hat{\varphi})$ is a $(1,1)$-form and $\Lambda(\alpha \wedge \hat{\varphi})$ is a $(2,0)+(0,2)$-form. Thus (9.1) becomes

$$
\begin{aligned}
0 & =\int_{M}|\varphi|^{2}\left(-\Lambda(\alpha \wedge \hat{\varphi})+\partial_{-} \hat{\varphi}\right) \wedge \omega \wedge \partial_{-} \hat{\varphi}=\int_{M}|\varphi|^{2} \omega \wedge \partial_{-} \hat{\varphi} \wedge \partial_{-} \hat{\varphi} \\
& =-\int_{M}|\varphi|^{2}\left|\partial_{-} \hat{\varphi}\right|^{2} \frac{\omega^{3}}{3 !} .
\end{aligned}
$$


Consequently we conclude that $\partial_{-} \hat{\varphi}=0$ and $d \hat{\varphi}=\omega \wedge \partial_{-} \hat{\varphi}=0$. In view of Lemma 7 , the almost-complex structure $J$ is integrable and the form $\varphi$ is harmonic. Now we use the integration by parts argument again to get

$$
\begin{aligned}
0 & =\int_{M} d \partial_{-}\left(|\varphi|^{2} \hat{\varphi}\right) \wedge|\varphi|^{2} \hat{\varphi}=-\int_{M} \omega \wedge \partial_{-}\left(|\varphi|^{2} \hat{\varphi}\right) \wedge \partial_{-}\left(|\varphi|^{2} \hat{\varphi}\right) \\
& =-\int_{M}|\varphi|^{4}|\Lambda(\alpha \wedge \hat{\varphi})|^{2} \frac{\omega^{3}}{3 !} .
\end{aligned}
$$

So we deduce that $\Lambda(\alpha \wedge \hat{\varphi})=0$, hence $\alpha \wedge \hat{\varphi}=0$ and $\alpha=0$, so $|\varphi|$ is a constant. Q.E.D.

\subsection{Integrable almost-complex structures: proof of Theorem 9}

Next, we give the proof of Theorem 9. The following identity for any smooth function $f$ and any differential form is well-known:

$$
d^{\dagger}(f \mu)=f d^{\dagger} \mu-\iota_{\nabla f} \mu .
$$

Indeed, it can be quickly verified by using $d^{\dagger} \mu=-\iota_{k}\left(\nabla^{k} \mu\right)$.

Back to the proof of Theorem 9, we apply Theorem 1 and the identity (9.5) to rewrite the Type IIA flow without sources as

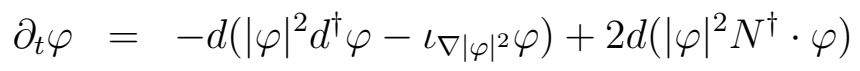

$$
\begin{aligned}
& =\mathcal{L}_{\nabla|\varphi|^{2}} \varphi-d\left(|\varphi|^{2} d^{\dagger} \varphi\right)+2 d\left(|\varphi|^{2} N^{\dagger} \cdot \varphi\right) \text {. }
\end{aligned}
$$

On any orbit of the diffeomorphism group which contains a form $\varphi$ with an integrable almost-complex structure, we have $N=0$ and, in view of Lemma 17, $d^{\dagger} \varphi=0$. Thus the flow reduces to

$$
\partial_{t} \varphi=\mathcal{L}_{\nabla|\varphi|^{2}} \varphi
$$

and a solution is given by the reparametrizations of $\varphi$ along the time-dependent vector field $\nabla|\varphi|^{2}$. By the uniqueness part of Theorem 2, this is the unique solution.

We now re-express the Type IIA flow in an equivalent formulation, but with a fixed complex structure. For this, let $f_{t}$ be the flow generated by the time-dependent vector field $-\nabla|\varphi|^{2}$ in the sense that

$$
\frac{d}{d t} f_{t}(x)=-\nabla|\varphi|^{2}(t, x)
$$

for any $x \in X$ and time $t$. It follows that

$$
\frac{d}{d t}\left(f_{t}^{*} \varphi_{t}\right)=f_{t}^{*} \partial_{t} \varphi_{t}+f_{t}^{*} \mathcal{L}_{-\nabla|\varphi|^{2}} \varphi_{t}=0
$$


hence $f_{t}^{*} \varphi_{t} \equiv \varphi_{0}$ is a constant 3 -form on $X$. We see immediately that if we reparametrize $M$ by the time-dependent diffeomorphism $f_{t}$, then along the flow, the 3 -form $f_{t}^{*} \varphi_{t}$ and hence the complex structure $f_{t}^{*} J_{t}$ are fixed. In this new gauge, the Kähler metric $\omega_{t}=f_{t}^{*} \omega$ evolves by the equation

$$
\partial_{t} \omega_{t}=f_{t}^{*} \mathcal{L}_{-\nabla|\varphi|^{2}} \omega
$$

Notice that

$$
\mathcal{L}_{-\nabla|\varphi|^{2}} \omega=-d \iota_{\nabla|\varphi|^{2}} \omega=d J d|\varphi|^{2}=-d d^{c}|\varphi|^{2}
$$

so the flow of $\omega_{t}$ can be written as

$$
\partial_{t} \omega_{t}=-d d^{c}|\varphi|_{\omega_{t}}^{2}
$$

We remark that this equation can be viewed as a T-dual of the Anomaly flow for conformally Kähler data. In that case, we are given a fixed holomorphic $(3,0)$ form $\check{\Omega}$ on a Calabi-Yau threefold and the evolving Kähler metrics $\check{\omega}_{t}$ satisfy (see equation (4.10) in [20])

$$
\partial_{t} \check{\omega}_{t}=d d^{c}|\check{\Omega}|_{\check{\omega}_{t}}^{-2}
$$

We see that $\check{R}=|\check{\Omega}|_{\check{\omega}}^{-2}$ is exchanged with $1 / R=|\varphi|_{\omega}^{2}$.

This type of duality was observed in [20] on semi-flat Calabi-Yau threefolds. To connect with the work there, we can consider the conformally changed metric $\eta_{t}=|\varphi|_{\omega_{t}}^{-2} \omega_{t}$. It follows that $|\varphi|_{\eta_{t}}=|\varphi|_{\omega_{t}}^{4}$, hence $\eta_{t}$ satisfies the conformally balanced equation $d\left(|\varphi|_{\eta_{t}} \eta_{t}^{2}\right)=$ 0 since

$$
|\varphi|_{\eta_{t}} \eta_{t}^{2}=|\varphi|_{\omega_{t}}^{4}\left(|\varphi|_{\omega_{t}}^{-2} \omega_{t}\right)^{2}=\omega_{t}^{2}
$$

is closed. Moreover its evolution equation is

$$
\begin{aligned}
\partial_{t}\left(|\varphi|_{\eta_{t}} \eta_{t}^{2}\right) & =\partial_{t}\left(\omega_{t}^{2}\right)=2 \omega_{t} \wedge \partial_{t} \omega_{t} \\
& =2 \omega_{t} \wedge\left(-d d^{c}|\varphi|_{\omega_{t}}^{2}\right)=-4 i \partial \bar{\partial}\left(|\varphi|_{\omega_{t}}^{2} \omega_{t}\right) \\
& =-4 i \partial \bar{\partial}\left(|\varphi|_{\eta_{t}} \eta_{t}\right),
\end{aligned}
$$

which is exactly (up to a positive constant) the dual Anomaly flow in complex dimension 3 firstly introduced in [20]. Since we are in the conformally Kähler case, by the results of [20], we know the flow (9.11) is equivalent to the inverse MA-flow introduced by Cao-Keller [10] and Collins-Hisamoto-Takahashi [7], which converges to the unique Ricci-flat Kähler metric in the cohomology class $\left[\omega_{0}\right]$.

\subsection{Symplectic manifolds with non-integrable almost complex structures}

Next, we work out the Type IIA flow on some model symplectic manifolds with nonintegrable almost-complex structures, more specifically tori, symplectic half-flat manifolds, and nilmanifolds. 


\subsubsection{The Type IIA flow on a torus}

Consider the 6 -torus $M=(\mathbf{R} / \mathbf{Z})^{6}$, with coordinates $\left\{x^{j}\right\}_{j=1}^{6}$ and the standard symplectic form $\omega=d x^{12}+d x^{34}+d x^{56}$. Let $\alpha, \beta, \gamma, \delta: \mathbf{R} / \mathbf{Z} \rightarrow \mathbf{R}$ be smooth functions depending only on the variable $x^{1}$. Consider

$$
\varphi=e^{\alpha} d x^{135}-e^{\beta} d x^{146}-d x^{245}-d x^{236}+\gamma d x^{136}+\delta d x^{145}
$$

Clearly $\varphi$ is closed and primitive. It is straightforward to find out that

$$
|\varphi|^{2}=2 \sqrt{4 e^{\alpha+\beta}-(\gamma-\delta)^{2}}
$$

and

$$
\begin{aligned}
\hat{\varphi}= & \frac{2}{|\varphi|^{2}}\left(-e^{\alpha}(\gamma+\delta) d x^{135}+\left(2 e^{\alpha+\beta}-\gamma(\gamma-\delta)\right) d x^{136}+\left(2 e^{\alpha+\beta}+\delta(\gamma-\delta)\right) d x^{145}\right. \\
& \left.+e^{\beta}(\gamma+\delta) d x^{146}+2 e^{\alpha} d x^{235}+(\gamma-\delta) d x^{236}-(\gamma-\delta) d x^{245}-2 e^{\beta} d x^{246}\right)
\end{aligned}
$$

Consequently we see that

$$
\begin{aligned}
d\left(|\varphi|^{2} \hat{\varphi}\right) & =2 d x^{12} \wedge\left(2\left(e^{\alpha}\right)^{\prime} d x^{35}+(\gamma-\delta)^{\prime}\left(d x^{36}-d x^{45}\right)-2\left(e^{\beta}\right)^{\prime} d x^{46}\right), \\
\Lambda d\left(|\varphi|^{2} \hat{\varphi}\right) & =2\left(2\left(e^{\alpha}\right)^{\prime} d x^{35}+(\gamma-\delta)^{\prime}\left(d x^{36}-d x^{45}\right)-2\left(e^{\beta}\right)^{\prime} d x^{46}\right), \\
d \Lambda d\left(|\varphi|^{2} \hat{\varphi}\right) & =4\left(e^{\alpha}\right)^{\prime \prime} d x^{135}+2(\gamma-\delta)^{\prime \prime}\left(d x^{136}-d x^{145}\right)-4\left(e^{\beta}\right)^{\prime \prime} d x^{146} .
\end{aligned}
$$

So the Type IIA flow in this case reduces to

$$
\begin{array}{ll}
\partial_{t}\left(e^{\alpha}\right)=4\left(e^{\alpha}\right)^{\prime \prime}, & \partial_{t}\left(e^{\beta}\right)=4\left(e^{\beta}\right)^{\prime \prime}, \\
\partial_{t} \gamma=2(\gamma-\delta)^{\prime \prime}, & \partial_{t} \delta=-2(\gamma-\delta)^{\prime \prime}
\end{array}
$$

For calculations, it is convenient to introduce $a=2 e^{\alpha}, b=2 e^{\beta}, c=\gamma-\delta, d=\gamma+\delta$ and

$$
|\varphi|^{2}=2 \sqrt{4 e^{\alpha+\beta}-(\gamma-\delta)^{2}}=2 \sqrt{a b-c^{2}} .
$$

It follows that $d$ is a constant along the flow, while $a, b, c$ satisfy the standard heat equation:

$$
\partial_{t}\left[\begin{array}{ll}
a & c \\
c & b
\end{array}\right]=4\left[\begin{array}{ll}
a & c \\
c & b
\end{array}\right]^{\prime \prime},
$$

Obviously the matrix $\left[\begin{array}{ll}a & c \\ c & b\end{array}\right]$ converges to a constant matrix as $t$ goes to infinity. Moreover along the flow the positive-definiteness is preserved and the limiting matrix is also positive definite. Thus $\varphi_{t}$ converges to a positive primitive harmonic form. 
Now let us analyze the behavior of $|N|^{2}$ along the flow. The easiest way to find $|N|^{2}$ is to use (7.28), which says that

$$
|N|^{2}=e^{-u} \partial_{t} u-\left(\Delta u+2|d u|^{2}\right) .
$$

The key is to compute $\Delta u$. Observe that the metric $g$ can be expressed as

$$
g=e^{-u}\left[\begin{array}{cccccc}
a b+d^{2}-c^{2} & -2 d & & & & \\
-2 d & 4 & & & & \\
& & 2 a & -2 c & & \\
& & -2 c & 2 b & & \\
& & & & 2 a & 2 c \\
& & & & 2 c & 2 b
\end{array}\right]
$$

As $\Delta u=g^{i j}\left(u_{i j}-\Gamma_{i j}^{k} u_{k}\right)=g^{11} u^{\prime \prime}-g^{i j} \Gamma_{i j}^{1} u^{\prime}$, and the Christoffel symbol term can be simplified to

$$
\begin{aligned}
g^{i j} \Gamma_{i j}^{1}= & \frac{1}{2} g^{i j} g^{1 l}\left(\partial_{i} g_{j l}+\partial_{j} g_{i l}-\partial_{l} g_{i j}\right) \\
= & g^{1 j} g^{1 l} g_{j l}^{\prime}-\frac{1}{2} g^{11} g^{i j} g_{i j}^{\prime} \\
= & \frac{1}{2}\left(g^{11}\right)^{2} g_{11}^{\prime}+g^{11} g^{12} g_{12}^{\prime}+\left(\left(g^{12}\right)^{2}-\frac{1}{2} g^{11} g^{22}\right) g_{22}^{\prime} \\
& -g^{11}\left(g^{33} g_{33}^{\prime}+2 g^{34} g_{34}^{\prime}+g^{44} g_{44}^{\prime}\right) \\
= & 8 e^{-3 u}\left(4 u^{\prime}\left(a b-c^{2}\right)-\left(a^{\prime} b+a b^{\prime}-2 c c^{\prime}\right)\right) \\
= & 8 e^{-3 u} v^{\prime},
\end{aligned}
$$

where $v=a b-c^{2}$ and $u=\log 2+\frac{1}{2} \log v$. Therefore

$$
\Delta u=4 e^{-u} u^{\prime \prime}-8 e^{-3 u} u^{\prime} v^{\prime}=4 e^{-u}\left(u^{\prime \prime}-\left(u^{\prime}\right)^{2}\right)
$$

Consequently

$$
\begin{aligned}
|N|^{2} & =e^{-u} \partial_{t} u-\left(\Delta u+2|d u|^{2}\right) \\
& =2 e^{-u} \frac{a^{\prime \prime} b+a b^{\prime \prime}-2 c c^{\prime \prime}}{v}-4 e^{-u}\left(u^{\prime \prime}+\left(u^{\prime}\right)^{2}\right) \\
& =16 e^{-5 u}\left(a b\left(2 c^{\prime}-\frac{c b^{\prime}}{b}-\frac{c a^{\prime}}{a}\right)^{2}+\frac{a b-c^{2}}{a b}\left(a b^{\prime}-a^{\prime} b\right)^{2}\right) .
\end{aligned}
$$

This calculation suggests that $J$ is integrable if and only if $a, b, c$ are proportional to each other. In summary we have proved

Proposition 8 Under our ansatze (9.14), the Type IIA flow on $(\mathbf{R} / \mathbf{Z})^{6}$ reduces to the standard heat equation on $\mathbf{R} / \mathbf{Z}$. If initially $\varphi$ is of the form (9.14) whose associated almost complex structure is not integrable, the Type IIA flow still converges to Kähler Calabi-Yau geometry. 


\subsubsection{The Type IIA flow on homogeneous symplectic half-flat manifolds}

Because of Theorem 8 , the convergence of the Type IIA flow is only possible when the underlying manifold is Kähler. We shall see in this subsection and the next that the Type IIA flow can be used to find optimal almost complex structures compatible with a given symplectic form, even when the underlying manifold does not admit any Kähler structure.

In order to run the Type IIA flow, we first need compact symplectic 6-manifolds with Type IIA structures. A special case of Type IIA structures can be found on the so-called symplectic half-flat manifolds (firstly introduced by de Bartolomeis [12], also known as special generalized Calabi-Yau manifolds [13]). In our terminology, a symplectic halfflat manifold is simply a symplectic manifold with Type IIA structure $(M, \omega, \varphi)$ and the extra condition that $|\varphi|^{2}$ is constant. Many compact symplectic half-flat manifolds can be constructed as quotients of Lie groups by co-compact lattices, where all the structures are homogeneous under the natural group action. Therefore we shall call symplectic halfflat manifolds constructed in this way homogeneous. It is clear that for homogeneous symplectic half-flat manifolds, their geometry up to covering is fully characterized by the underlying Lie algebra, or equivalently the exterior differential system defined by invariant 1 -forms. Moreover, homogeneous symplectic half-flat structures have been fully classified by [11] and [22] when the Lie group is nilpotent or solvable respectively.

It is clear that if we run the Type IIA flow on a homogeneous symplectic half-flat manifold with homogeneous initial data, the homogeneity is preserved and the Type IIA flow reduces to a polynomial ODE system. Moreover, in the homogeneous setting, the function $u$ and $|N|^{2}$ are constants on the manifold, therefore we have the following monotonicity formulas

Proposition 9 Along the Type IIA flow on homogenous symplectic half-flat manifolds, the following monotonicity formulae hold

$$
\begin{aligned}
\partial_{t} u & =e^{u}|N|^{2} \geq 0, \\
\partial_{t}|N|^{2} & =-2 e^{u}\left|\left(R^{-J}\right)_{i j}\right|^{2} \leq 0 .
\end{aligned}
$$

Proof: The first formula follows directly from (7.28). For the second formula, we note that Blair-Ianus [4 proved that

$$
\partial_{t} \int_{M}|N|^{2} \frac{\omega^{3}}{3 !}=\int_{M}\left(\partial_{t} g_{i j},\left(R^{-J}\right)_{i j}\right) \frac{\omega^{3}}{3 !} .
$$

In our case $u$ is a constant, hence (7.27) becomes $\partial_{t} g_{i j}=-2 e^{u}\left(R^{-J}\right)_{i j}$, and (9.25) simplifies to

$$
\partial_{t} \int_{M}|N|^{2} \frac{\omega^{3}}{3 !}=-2 \int_{M} e^{u}\left|\left(R^{-J}\right)_{i j}\right|^{2} \frac{\omega^{3}}{3 !} .
$$

As everything is homogeneous, so all the scalars must be constant, consequently (9.26) still holds without integration, and (9.24) is proved. Q.E.D. 
Corollary 2 Let $(M, \omega)$ be a compact 6-dimensional homogeneous symplectic manifold. If $(M, \omega)$ admits a homogeneous symplectic half-flat structure $\left(M, \omega, \varphi_{0}\right)$ with which the Type IIA flow exists for all time, then there exist homogenous almost complex structures compatible with $\omega$ and with arbitrary small Nijenhuis tensor.

Proof of the Corollary: We run the Type IIA flow with initial data $\varphi_{0}$. By monotonicity formulas above, we know that

$$
\begin{aligned}
\frac{d}{d t} e^{-u} & =-|N|^{2} \leq 0, \\
\frac{d^{2}}{d t^{2}} e^{-u} & =2 e^{u}\left|\left(R^{-J}\right)_{i j}\right|^{2} \geq 0 .
\end{aligned}
$$

So $e^{-u}$ is a monotone non-increasing and convex function with lower bound. If the flow exists for all time, then we must have $\lim _{t \rightarrow \infty}|N|^{2}=-\lim _{t \rightarrow \infty} \frac{d}{d t} e^{-u}=0$, as was to be shown. Q.E.D.

\section{The Type IIA flow on a nilmanifold}

Now let us consider some explicit examples.

Consider the homogeneous symplectic half-flat structure in [13, Example 5.2], where the Lie algebra of the nilpotent Lie group is characterized by invariant 1 -forms $\left\{e^{1}, \ldots, e^{6}\right\}$ satisfying

$$
\begin{aligned}
& d e^{1}=d e^{2}=d e^{3}=d e^{5}=0, \\
& d e^{4}=e^{15}, \quad d e^{6}=e^{13} .
\end{aligned}
$$

Clearly $\omega=e^{12}+e^{34}+e^{56}$ defines an invariant symplectic structure. Moreover, this nilpotent Lie group admits co-compact lattices so all the constructions descend to compact nilmanifolds. Consider the ansatze

$$
\varphi=\varphi_{a, b}=(1+a) e^{135}-e^{146}-e^{245}-e^{236}+b\left(e^{134}-e^{156}\right),
$$

it is straightforward to check that $\varphi_{a, b}$ is primitive and closed for any $a, b$. The positivity condition for $\varphi_{a, b}$ is that $\frac{1}{16}|\varphi|^{4}=1+a-b^{2}>0$. By straightforward calculations, we get

$$
\hat{\varphi}=4|\varphi|^{-2}\left(\left(1+a-b^{2}\right) e^{1} \wedge\left(e^{36}+e^{45}\right)+e^{2} \wedge\left(b e^{34}+(1+a) e^{35}-e^{46}-b e^{56}\right)\right) .
$$

It follows that

$$
\begin{aligned}
& d\left(|\varphi|^{2} \hat{\varphi}\right)=4 e^{12}\left(e^{34}+2 b e^{35}-e^{56}\right), \\
& \Lambda d\left(|\varphi|^{2} \hat{\varphi}\right)=4\left(e^{34}+2 b e^{35}-e^{56}\right), \quad d \Lambda d\left(|\varphi|^{2} \hat{\varphi}\right)=8 e^{135} .
\end{aligned}
$$


Therefore under our ansatze the Type IIA flow reduces to the following ODE system

$$
\dot{a}(t)=8, \quad \dot{b}(t)=0 .
$$

Hence the unique solution to the Type IIA flow is

$$
\varphi(t)=\left(1+a_{0}+8 t\right) e^{135}-e^{146}-e^{245}-e^{236}+b_{0}\left(e^{134}-e^{156}\right),
$$

which exists for all time $t \geq 0$.

One can easily verify that $\lim _{t \rightarrow \infty} J_{t}$ does not exist and

$$
|N|^{2}=\left(1+a-b^{2}\right)^{-3 / 2}=\left(1+a_{0}+8 t-b_{0}^{2}\right)^{-3 / 2}
$$

is decreasing to zero as $t \rightarrow \infty$. This is an explicit example where Corollary 2 applies.

\section{The Type IIA flow on a solvmanifold}

Consider the symplectic half-flat structure on the solvmanifold $M$ constructed by Tomassini and Vezzoni in [46, Theorem 3.5]. The geometry of this solvmanifold is characterized by invariant 1-forms $\left\{e^{j}\right\}_{j=1}^{6}$ satisfying

$$
\begin{aligned}
& d e^{1}=-\lambda e^{15}, \quad d e^{2}=\lambda e^{25}, \quad d e^{3}=-\lambda e^{36}, \\
& d e^{4}=\lambda e^{46}, \quad d e^{5}=0, \quad d e^{6}=0,
\end{aligned}
$$

where $\lambda=\log \frac{3+\sqrt{5}}{2}$. One can easily check that $\omega=e^{12}+e^{34}+e^{56}$ is an invariant symplectic form on $M$. A particular symplectic half-flat structure on $M$ takes the form

$$
\begin{aligned}
\varphi & =\frac{\sqrt{2}}{2}\left(e^{135}+e^{136}+e^{145}-e^{146}+e^{235}-e^{236}-e^{245}-e^{246}\right) \\
& =\frac{\sqrt{2}}{2 \lambda} d\left(e^{13}+e^{14}-e^{23}+e^{24}\right),
\end{aligned}
$$

so $[\varphi]=0 \in H^{3}(M ; \mathbf{R})$.

Consider the ansatze

$$
\varphi=\alpha\left(e^{135}+e^{136}\right)+\beta\left(e^{145}-e^{146}\right)+\gamma\left(e^{235}-e^{236}\right)-\delta\left(e^{245}+e^{246}\right) .
$$

A direct calculation gives

$$
|\varphi|^{2} \hat{\varphi}=8\left(-\alpha \beta \gamma\left(e^{135}-e^{136}\right)+\alpha \beta \delta\left(e^{145}+e^{146}\right)+\alpha \gamma \delta\left(e^{235}+e^{236}\right)+\beta \gamma \delta\left(e^{245}-e^{246}\right)\right) .
$$

The nondegenerate condition is that $|\varphi|^{4}=64 \alpha \beta \gamma \delta>0$. It follows that

$$
\begin{aligned}
d\left(|\varphi|^{2} \hat{\varphi}\right)= & 16 \lambda\left(\alpha \beta \gamma e^{1356}+\alpha \beta \delta e^{1456}-\alpha \gamma \delta e^{2356}+\beta \gamma \delta e^{2456}\right) \\
\Lambda d\left(|\varphi|^{2} \hat{\varphi}\right)= & 16 \lambda\left(\alpha \beta \gamma e^{13}+\alpha \beta \delta e^{14}-\alpha \gamma \delta e^{23}+\beta \gamma \delta e^{24}\right) \\
d \Lambda d\left(|\varphi|^{2} \hat{\varphi}\right)= & 16 \lambda^{2}\left(\alpha \beta \gamma\left(e^{135}+e^{136}\right)+\alpha \beta \delta\left(e^{145}-e^{146}\right)\right. \\
& \left.+\alpha \gamma \delta\left(e^{235}-e^{236}\right)-\beta \gamma \delta\left(e^{245}+e^{246}\right)\right)
\end{aligned}
$$


After time rescaling, the Type IIA flow under our ansatze reduces to

$$
\begin{array}{ll}
\partial_{t} \alpha=\alpha \beta \gamma, & \partial_{t} \beta=\alpha \beta \delta, \\
\partial_{t} \gamma=\alpha \gamma \delta, & \partial_{t} \delta=\beta \gamma \delta .
\end{array}
$$

It is easy to see that there exist time-independent nonzero constants $C_{1}$ and $C_{2}$ such that $\alpha(t)=C_{1} \delta(t)$ and $\beta(t)=C_{2} \gamma(t)$. The ODE system simplifies to

$$
\partial_{t} \gamma=C_{1} \gamma \delta^{2}, \quad \partial_{t} \delta=C_{2} \gamma^{2} \delta
$$

Integrate these equations we know there is a constant $C$ such that $C_{2} \gamma^{2}-C_{1} \delta^{2}=C$, hence

$$
\partial_{t} \gamma=\gamma\left(C_{2} \gamma^{2}-C\right)
$$

One can solve explicitly

$$
\gamma^{2}(t)=\frac{C \gamma_{0}^{2}}{C_{2} \gamma_{0}^{2}\left(1-e^{2 C t}\right)+C e^{2 C t}}, \quad \delta^{2}(t)=\frac{C \delta_{0}^{2} e^{2 C t}}{C_{1} \delta_{0}^{2}+C-C_{1} \delta_{0}^{2} e^{2 C t}} .
$$

Assuming $\varphi$ is initially positive, we know that $C_{1}, C_{2}$ are positive constants and $\gamma_{0}, \delta_{0}$ are initial values of $\gamma$ and $\delta$ satisfying $\beta \gamma-\alpha \delta=C_{2} \gamma_{0}^{2}-C_{1} \delta_{0}^{2}=C$. When $C=0$, the above formula should be understood as

$$
\gamma^{2}=\frac{\gamma_{0}^{2}}{1-2 C_{2} \gamma_{0}^{2} t}, \quad \delta^{2}=\frac{\delta_{0}^{2}}{1-2 C_{1} \delta_{0}^{2} t}
$$

From the above explicit formulas, we can deduce that, no matter what $C$ is, the flow has finite time singularity. A more symmetric expression for the solution (without time rescaling) is

$$
\begin{aligned}
& \alpha(t)=\alpha_{0} \sqrt{\frac{\left(\beta_{0} \gamma_{0}-\alpha_{0} \delta_{0}\right) e^{32 \lambda^{2} \beta_{0} \gamma_{0} t}}{\beta_{0} \gamma_{0} e^{32 \lambda^{2} \alpha_{0} \delta_{0} t}-\alpha_{0} \delta_{0} e^{32 \lambda^{2} \beta_{0} \gamma_{0} t}}}, \quad \beta(t)=\beta_{0} \sqrt{\frac{\left(\beta_{0} \gamma_{0}-\alpha_{0} \delta_{0}\right) e^{32 \lambda^{2} \alpha_{0} \delta_{0} t}}{\beta_{0} \gamma_{0} e^{32 \lambda^{2} \alpha_{0} \delta_{0} t}-\alpha_{0} \delta_{0} e^{32 \lambda^{2} \beta_{0} \gamma_{0} t}}}, \\
& \gamma(t)=\gamma_{0} \sqrt{\frac{\left(\beta_{0} \gamma_{0}-\alpha_{0} \delta_{0}\right) e^{32 \lambda^{2} \alpha_{0} \delta_{0} t}}{\beta_{0} \gamma_{0} e^{32 \lambda^{2} \alpha_{0} \delta_{0} t}-\alpha_{0} \delta_{0} e^{32 \lambda^{2} \beta_{0} \gamma_{0} t}}}, \quad \delta(t)=\delta_{0} \sqrt{\frac{\left(\beta_{0} \gamma_{0}-\alpha_{0} \delta_{0}\right) e^{32 \lambda^{2} \beta_{0} \gamma_{0} t}}{\beta_{0} \gamma_{0} e^{32 \lambda^{2} \alpha_{0} \delta_{0} t}-\alpha_{0} \delta_{0} e^{32 \lambda^{2} \beta_{0} \gamma_{0} t}}},
\end{aligned}
$$

and in the critical case when $\alpha_{0} \delta_{0}=\beta_{0} \gamma_{0}=S>0$, one has

$$
\begin{array}{ll}
\alpha(t)=\frac{\alpha_{0}}{\sqrt{1-32 \lambda^{2} S t}}, & \beta(t)=\frac{\beta_{0}}{\sqrt{1-32 \lambda^{2} S t}}, \\
\gamma(t)=\frac{\gamma_{0}}{\sqrt{1-32 \lambda^{2} S t}}, & \delta(t)=\frac{\delta_{0}}{\sqrt{1-32 \lambda^{2} S t}} .
\end{array}
$$

From these explicit expressions, we see that the maximal existence time $T$ is given by

$$
T=\frac{1}{32 \lambda^{2}} \frac{\log \left(\alpha_{0} \delta_{0}\right)-\log \left(\beta_{0} \gamma_{0}\right)}{\alpha_{0} \delta_{0}-\beta_{0} \gamma_{0}} .
$$


All of $\alpha, \beta, \gamma, \delta$ tend to infinity as $t \rightarrow T$, therefore $|\varphi|^{2}=8(\alpha \beta \gamma \delta)^{1 / 2} \rightarrow \infty$. To compute $|N|^{2}$, the quickest way is to use (9.23):

$$
\begin{aligned}
|N|^{2} & =e^{-u} \partial_{t} u=-\partial_{t} e^{-u}=\frac{(\alpha \beta \gamma \delta)^{-3 / 2}}{16} \partial_{t}(\alpha \beta \gamma \delta)=2 \lambda^{2} \frac{\alpha \delta+\beta \gamma}{(\alpha \beta \gamma \delta)^{1 / 2}} \\
& =\frac{2 \lambda^{2}}{\left(\alpha_{0} \beta_{0} \gamma_{0} \delta_{0}\right)^{1 / 2}}\left(\alpha_{0} \delta_{0} e^{16 \lambda^{2}\left(\beta_{0} \gamma_{0}-\alpha_{0} \delta_{0}\right) t}+\beta_{0} \gamma_{0} e^{-16 \lambda^{2}\left(\beta_{0} \gamma_{0}-\alpha_{0} \delta_{0}\right) t}\right) \\
& \geq 4 \lambda^{2} .
\end{aligned}
$$

Now let us analyze the behavior of $\varphi$ in detail. We shall see that the Type IIA flow naturally leads us to optimal almost-complex structures compatible with $\omega$.

1. No matter what the integral constant $C=\beta_{0} \gamma_{0}-\alpha_{0} \delta_{0}$ is, the flow of $\varphi$ blows up when $t \rightarrow T$, and the same is true for the metric $\tilde{g}$. However the expressions of $g, J$, and $N$ extend smoothly to $t=T$. In fact, the limit $|\varphi|^{-1} \varphi$ as $t \rightarrow T$ exists.

2. In the critical case $C=\beta_{0} \gamma_{0}-\alpha_{0} \delta_{0}=0$, the flow of $\varphi$ (as well as $\tilde{g}$ ) is a self-expander in the sense that

$$
\varphi(t)=\frac{\varphi_{0}}{\sqrt{1-32 \lambda^{2} S t}}
$$

for a positive constant $S$ determined by initial data. In this case, all of $g, J$ and $N$ are stationary with $|N|^{2}=4 \lambda^{2}$. In fact such $J$ provide examples of harmonic almost-complex structures in the sense of Blair-Ianus [4], namely almost-complex structures compatible with $\omega$ and satisfying

$$
\left(R^{-J}\right)_{i j}=\frac{1}{2}\left(R_{i j}-R_{J i, J j}\right)=0 .
$$

These harmonic almost-complex structures are critical points of the energy functional studied by Blair-Ianus [4] and Lê-Wang [33].

3. When the integral constant $C=\beta_{0} \gamma_{0}-\alpha_{0} \delta_{0}$ is not zero, all of $g, J$ and $N$ are evolving. When $t$ approaches $T$, the $\operatorname{limit}_{\lim _{t \rightarrow T}} J(t)$ exists and is a harmonic almost-complex structure, which is also a minimizer of $|N|^{2}$ among all almost complex structures associated to our ansatze (9.32).

Acknowledgements The authors would like to thank F. Fong, R. Rosso, A. Sun, and L.-S. Tseng for very helpful communications. The second-named author would also like to thank the Galileo Galilei Institute for Theoretical Physics, the Banff Research Station, and the American Institute of Mathematics for their hospitality. 


\section{References}

[1] K. Becker, M. Becker, and J.H. Schwarz, String Theory and M-Theory: A Modern Introduction, Cambridge University Press, 2006.

[2] L. Bedulli and L. Vezzoni, Stability of flows of closed forms, Adv. Math. 364 (2020), 107030, 29 pp..

[3] D.E. Blair, The "total scalar curvature" as a symplectic invariant and related results, Proceedings of the 3rd Congress of Geometry (Thessaloniki, 1991), 79-83, Aristotle Univ., 1992.

[4] D.E. Blair and S. Ianus, Critical associated metrics on symplectic manifolds, Nonlinear Problems in Geometry, 23-29, Contemp. Math., 51, AMS, 1986.

[5] R.L. Bryant, Some remarks on $G_{2}$-structures, Proceedings of Gökova Geometry-Topology Conference 2005, 75-109, International Press, 2006.

[6] R.L. Bryant and Xu, Laplacian flow for closed $G_{2}$-structures: short time behavior, arXiv: 1101.2004.

[7] T. Collins, T. Hisamoto and R. Takahashi, The inverse Monge-Ampère flow and applications to Kähler-Einstein metrics, arXiv: 1712.01685.

[8] P. Candelas, G. Horowitz, A.E. Strominger, and E. Witten, Vacuum configurations for superstrings, Nuclear Phys. B 258 (1985), no. 1, 46-74.

[9] H.D. Cao, Deformation of Kähler metrics to Kähler-Einstein metrics on compact Kähler manifolds, Invent. Math. 81 (1985), no. 2, 359-372.

[10] H.D. Cao and J. Keller, About the Calabi problem: a finite-dimensional approach, J. Eur. Math. Soc. 15 (2013), no. 3, 1033-1065.

[11] D. Conti and A. Tomassini, Special symplectic six-manifolds, Q. J. Math. 58 (2007), no. 3, 297-311.

[12] P. de Bartholomeis, Geometric structures on moduli spaces of special Lagrangian submanifolds, Ann. Mat. Pura Appl. (4) 179 (2001), 361-382.

[13] P. de Bartholomeis and A. Tomassini, On the Maslov index of Lagrangian submanifolds of generalized Calabi-Yau manifolds, Internat. J. Math. 17 (2006), no. 8, 921-947.

[14] D.M. DeTurck, Deforming metrics in the direction of their Ricci tensors, J. Differential Geom. 18 (1983), no. 1, 157-162.

[15] T. Fei, Stable forms, vector cross products and their applications in geometry, arXiv: 1504.02807.

[16] T. Fei, On the geometry of the Strominger system, Massachusetts Institute of Technology Ph.D. Thesis, 2016.

[17] T. Fei and D.H. Phong, Unification of the Kähler-Ricci and Anomaly flows, Differential Geometry, Calabi-Yau Theory, and General Relativity, 89-104, Surv. Differ. Geom., 22, International Press, 2018 .

[18] T. Fei, Z.J. Huang, and S. Picard, A construction of infinitely many solutions to the Strominger system, arXiv: 1703.10067 .

[19] T. Fei, D.H. Phong, S. Picard, and X.W. Zhang, Estimates for a geometric flow for the Type IIB string, arXiv: 2004.14529. 
[20] T. Fei and S. Picard, Anomaly flow and T-duality, arXiv: 1903.08768.

[21] T. Fei and S.-T. Yau, Invariant solutions to the Strominger system on complex Lie groups and their quotients, Comm. Math. Phys. 338 (2015), no. 3, 1183-1195.

[22] M. Fernández, V. Manero, A. Otal, and L. Ugarte Symplectic half-flat solvmanifolds, Ann. Global Anal. Geom. 43 (2013), no. 4, 367-383.

[23] J.-X. Fu and S.-T. Yau, A Monge-Ampère-type equation motivated by string theory, Comm. Anal. Geom. 15 (2007), no. 1, 29-75.

[24] J.-X. Fu and S.-T. Yau, The theory of superstring with flux on non-Kähler manifolds and the complex Monge-Ampère equation, J. Differential Geom. 78 (2008), no. 3, 369-428.

[25] M. Garcia-Fernandez, Lectures on the Strominger system, Travaux mathématiques. Vol. XXIV, 7-61, Trav. Math., 24, University of Luxembourg, 2016.

[26] P. Gauduchon, Hermitian connections and Dirac operators, Boll. Un. Mat. Ital. B (7) 11 (1997), no. 2, suppl., 257-288.

[27] M. Grana, R. Minasian, M. Petrini, and A. Tomasiello, Generalized structures of $\mathcal{N}=1$ vacua, J. High Energy Phys. 11 (2005), 020.

[28] S.S. Gubser, Special holonomy in string theory and M-theory, Strings, Branes and Extra Dimensions: TASI 2001, 197-233, World Scientific, 2004.

[29] R.S. Hamilton, Three-manifolds and positive Ricci curvature, J. Differential Geom. 17 (1982), no. 2, 255-306.

[30] N.J. Hitchin, The geometry of three-forms in six dimensions, J. Differential Geom. 55 (2000), no. 3, 547-576.

[31] P. Horava and E. Witten, Heterotic and Type I string dynamics from eleven dimensions, Nuclear Phys. B 460 (1996), no. 3, 506-524.

[32] C.M. Hull, Supersymmetry with torsion and space-time supersymmetry, 1st Torino Meeting on Superunification and Extra Dimensions, 347-375, World Scientific, 1986.

[33] H.V. Lê and G.-F. Wang, Anti-complexified Ricci flow on compact symplectic manifolds, J. Reine Angew. Math. 530 (2001), 17-31.

[34] J.D. Lotay, Geometric flows of $G_{2}$ structures, Lectures and Surveys on $G_{2}$-Manifolds and Related Topics, 113-140, Fields Inst. Commun., 84, Springer, 2020.

[35] J.D. Lotay and Y. Wei, Laplacian flow for closed $G_{2}$ structures: Shi-type estimate, uniqueness and compactness, Geom. Funct. Anal. 27 (2017), no. 1, 165-233.

[36] V. Oproiu, A global invariant of the symplectic manifolds, An. Stiint. Univ. Al. I. Cuza Iasi Sect. I a Mat. 36 (1990), no. 4, 393-396.

[37] D.H. Phong, Geometric partial differential equations from unified string theories, arXiv: 1906.03693.

[38] D.H. Phong, S. Picard, and X.W. Zhang, Geometric flows and the Strominger system, Math. Z. 288 (2018), no. 1-2, 101-113 
[39] D.H. Phong, S. Picard, and X.W. Zhang, Anomaly flows, Comm. Anal. Geom. 26 (2018), no. 4, 955-1008.

[40] D.H. Phong, S. Picard, and X.W. Zhang, The Fu-Yau equation with negative slope parameter, Invent. Math. 209 (2017), no. 2, 541-576.

[41] D.H. Phong, S. Picard, and X.W. Zhang, The Anomaly flow and the Fu-Yau equation, Ann. PDE 4 (2018), no. 2, Paper No. 13, 60 pp.

[42] D.H. Phong, S. Picard, and X.W. Zhang, A flow of conformally balanced metrics with Kähler fixed points, Math. Ann. 374 (2019), no. 3-4, 2005-2040.

[43] S. Picard, and X.W. Zhang, Parabolic complex Monge-Ampère equations on compact Kähler manifolds, arXiv:1906.10235.

[44] A.E. Strominger, Superstrings with torsion, Nuclear Phys. B 274 (1986), no. 2, 253-284.

[45] A. Tomasiello, Reformulating supersymmetry with a generalized Dolbeault operator, J. High Energy Phys. 2008, no. 2, 010, 25 pp.

[46] A. Tomassini and L. Vezzoni, On symplectic half-flat manifolds, Manuscripta Math. 125 (2008), no. $4,515-530$.

[47] V. Tosatti, B. Weinkove, and S.-T. Yau, Taming symplectic forms and the Calabi-Yau equation, Proc. Lond. Math. Soc. (3) 97 (2008), no. 2, 401-424.

[48] P.K. Townsend, Four Lectures on M-theory, 1996 Summer School in High Energy Physics and Cosmology, 385-438, ICTP Ser. Theoret. Phys., 13, World Scientific, 1997.

[49] L.-S. Tseng and L.H. Wang, Hodge theory and symplectic boundary conditions, arXiv: 1409.8250.

[50] L.-S. Tseng and S.-T. Yau, Cohomology and Hodge theory on symplectic manifolds: I, J. Differential Geom. 91 (2012), no. 3, 383-416.

[51] L.-S. Tseng and S.-T. Yau, Cohomology and Hodge theory on symplectic manifolds: II, J. Differential Geom. 91 (2012), no. 3, 417-443.

[52] L.-S. Tseng and S.-T. Yau, Generalized cohomologies and supersymmetry, Commun. Math. Phys. 326 (2014), no. 3, 875-885.

[53] S.-T. Yau, On the Ricci curvature of a compact Kähler manifold and the complex Monge-Ampère equation. I., Comm. Pure Appl. Math. 31 (1978), no. 3, 339-411.

Department of Mathematics \& Computer Science, Rutgers, Newark, NJ 07102, USA teng.fei@rutgers.edu

Department of Mathematics, Columbia University, New York, NY 10027, USA phong@math.columbia.edu

Mathematics Department, University of British Columbia, Vancouver, BC V6T 1Z2, CAN spicard@math.ubc.ca

Department of Mathematics, University of California, Irvine, CA 92697, USA xiangwen@math.uci.edu 